Supporting Information:

\title{
Pd(II)-Catalyzed Asymmetric Annulation towards the Synthesis of 2,3-Disubstituted Chiral Indenols
}

\author{
Chang-Xue Gu, Wen-Wen Chen, ${ }^{\mathrm{a}, *}$ and Ming-Hua $\mathrm{Xu}^{\mathrm{a}, \mathrm{b} *}$ \\ ${ }^{a}$ State Key Laboratory of Drug Research, Shanghai Institute of Materia Medica, Chinese \\ Academy of Sciences, 555 Zuchongzhi Road, Shanghai 201203 \\ ${ }^{b}$ Shenzhen Grubbs Institute and Department of Chemistry, Southern University of Science \\ and Technology, 1088 Xueyuan Boulevard, Shenzhen 518055, China \\ E-mail: wenwen@shnu.edu.cn, xumh@sustech.edu.cn
}

\section{Table of contents}

1. HPLC charts of products $\mathbf{3}, \mathbf{5}$ and $\mathbf{6}$. . $\mathrm{S} 2$

2. Copies of ${ }^{1} \mathrm{H}$ NMR, ${ }^{13} \mathrm{C}$ NMR and ${ }^{19} \mathrm{~F}$ NMR spectra of products $\mathbf{3}, \mathbf{5}$ and $\mathbf{6} \ldots \ldots . . \mathrm{S} 31$

3. X-Ray structure of compound $\mathbf{3 k}$ S62 
1. HPLC charts of arylation products

(S)-2,3-diphenyl-1H-inden-1-ol (3a)

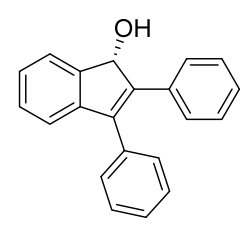

$3 a$

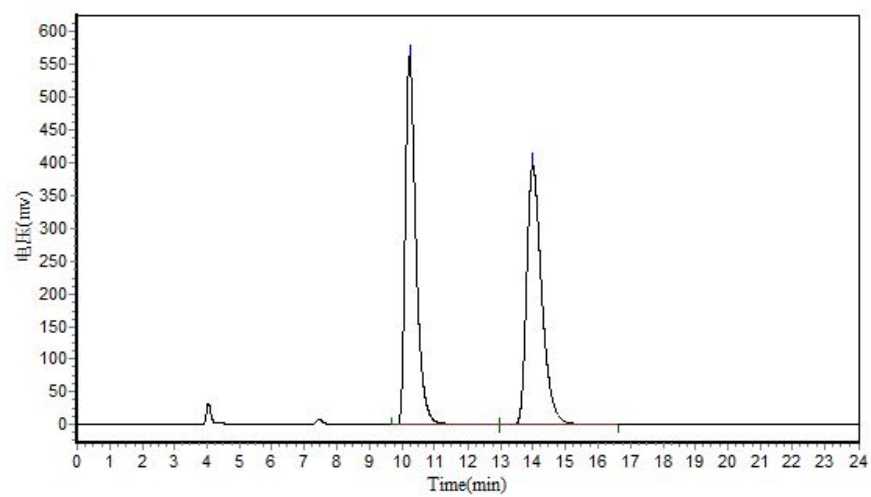

Results

\begin{tabular}{|c|c|c|c|c|c|}
\hline \multicolumn{6}{|c|}{ - } \\
\hline Peak No. & Peak ID & Ret Time & Height & Area & Conc. \\
\hline 1 & & 10.248 & 561655.313 & 12854595.000 & 50.0664 \\
\hline 2 & & 13.998 & 395707.563 & 12820499.000 & 49.9336 \\
\hline Total & & & 957362.875 & 25675094.000 & $100.000 \mathrm{C}$ \\
\hline
\end{tabular}

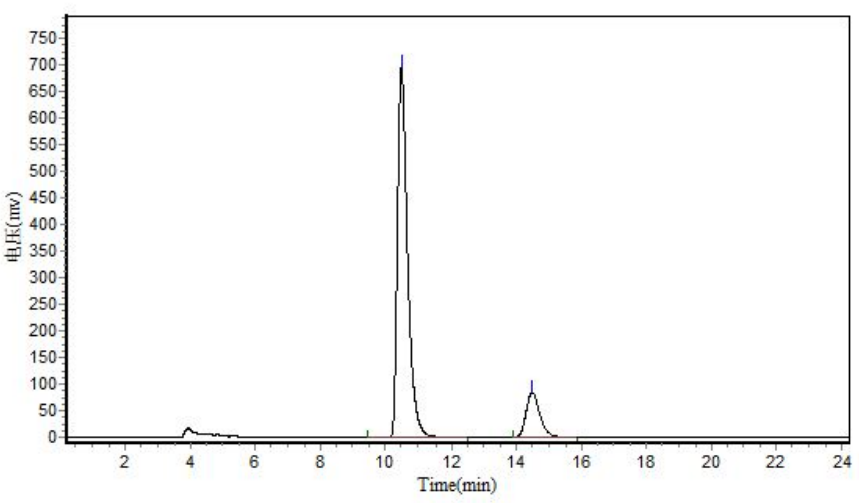

\begin{tabular}{|c|c|c|c|c|c|}
\hline \multicolumn{6}{|c|}{ Results } \\
\hline Peak No. & Peak ID & Ret Time & Height & Area & Conc. \\
\hline 1 & & 10.492 & 694677.813 & 15016484.000 & 85.5460 \\
\hline 2 & & 14.478 & 85261.297 & 2537221.000 & 14.4540 \\
\hline Total & & & 779939.109 & 17553705.000 & 100.0000 \\
\hline
\end{tabular}




\section{(S)-2,3-bis(4-methoxyphenyl)-1H-inden-1-ol (3b)}
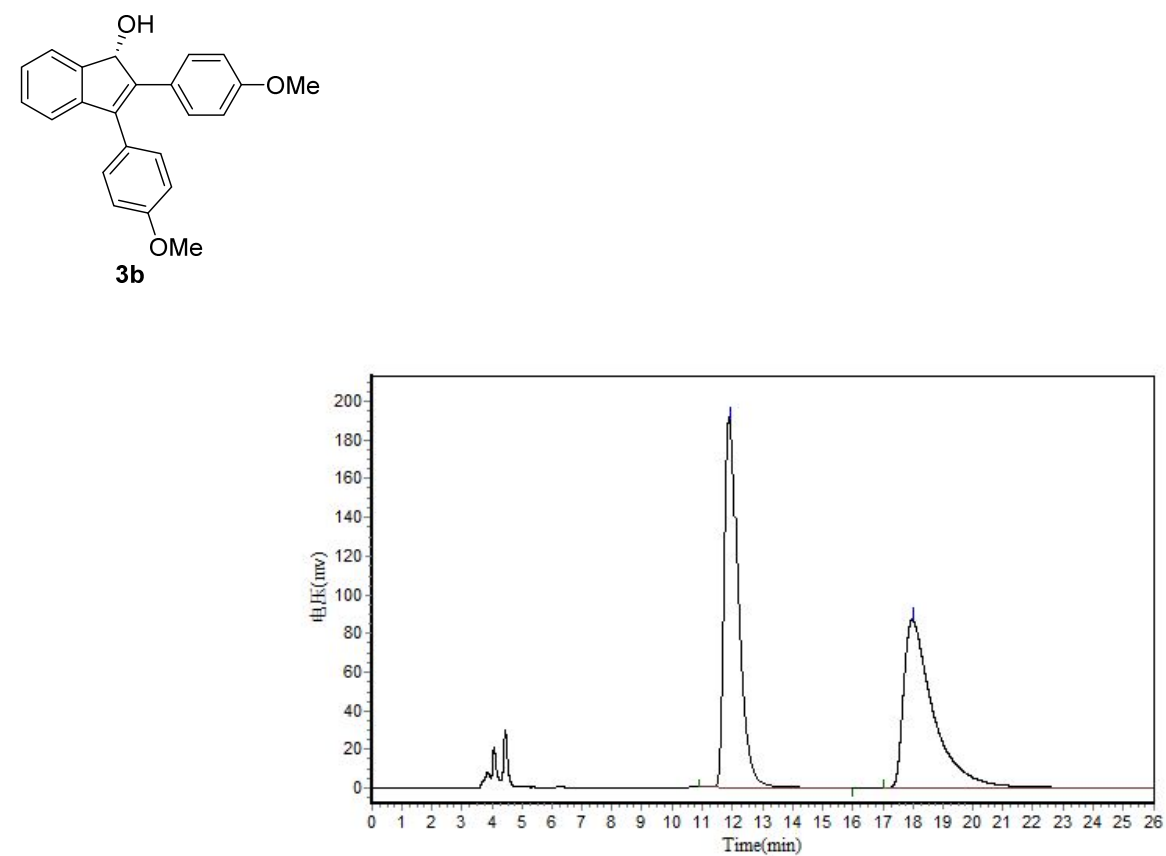

\begin{tabular}{|c|c|c|c|c|c|}
\hline \multicolumn{6}{|c|}{ Results } \\
\hline Peak No. & Peak ID & Ret Time & Height & Area & Conc. \\
\hline 1 & & 11.932 & 191239.125 & 5992210.000 & 49.6590 \\
\hline 2 & & 17.998 & 86923.867 & 6074510.000 & 50.3410 \\
\hline Total & & & 278162.992 & 12066720.000 & 100.0000 \\
\hline
\end{tabular}

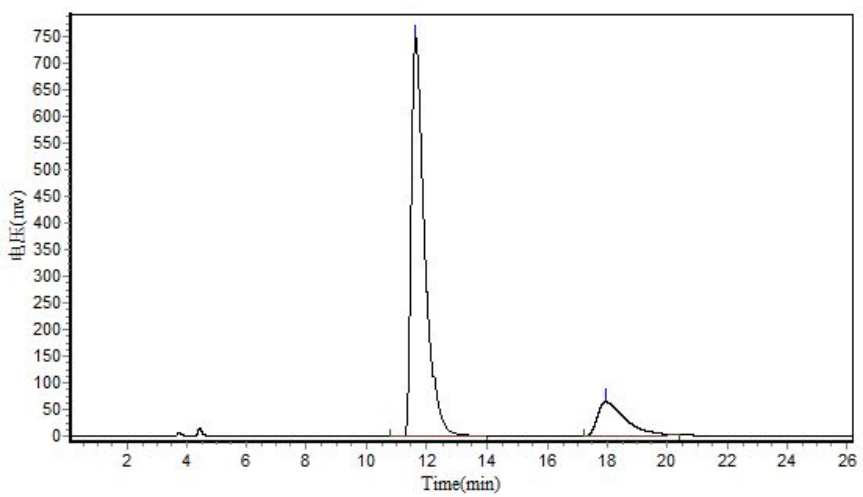

\begin{tabular}{|c|c|c|c|c|c|}
\hline \multicolumn{6}{|c|}{ Results } \\
\hline Peak No. & Peak ID & Ret Time & Height & Area & Conc. \\
\hline 1 & & 11.650 & 747618.375 & 22954160.000 & 85.0875 \\
\hline 2 & & 17.962 & 63254.285 & 4022975.500 & 14.9125 \\
\hline Total & & & $\$ 10872.660$ & 26977135.500 & 100.0000 \\
\hline
\end{tabular}




\section{(S)-2,3-di-p-tolyl-1H-inden-1-ol (3c)}
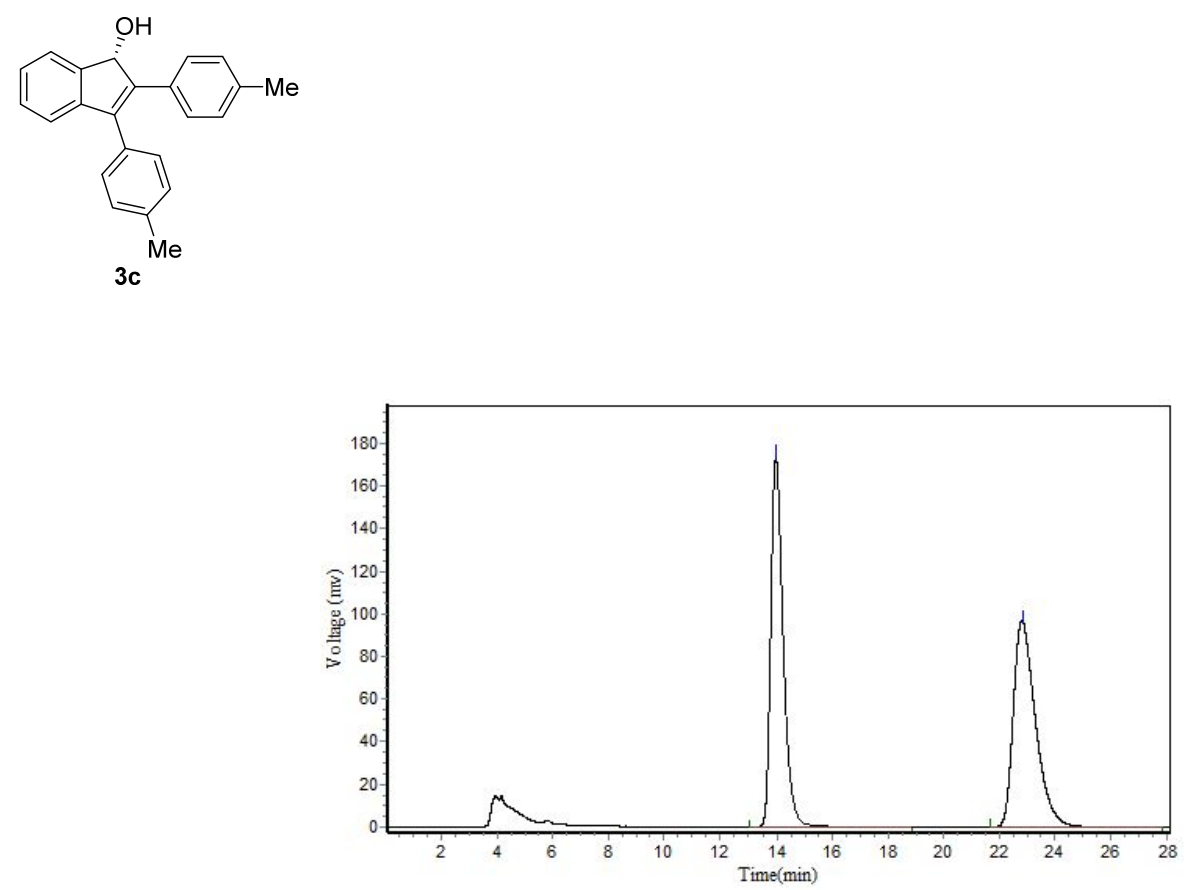

\begin{tabular}{|c|c|c|c|c|c|}
\hline \multicolumn{6}{|c|}{ Results } \\
\hline Peak No. & Peak ID & Ret Time & Height & Area & Conc. \\
\hline 1 & & 13.965 & 172922.172 & 5429119.000 & 50.0733 \\
\hline 2 & & 22.865 & 96879.492 & 5413224.500 & 49.9267 \\
\hline Total & & & 269801.664 & 10842343.500 & 100.0000 \\
\hline
\end{tabular}

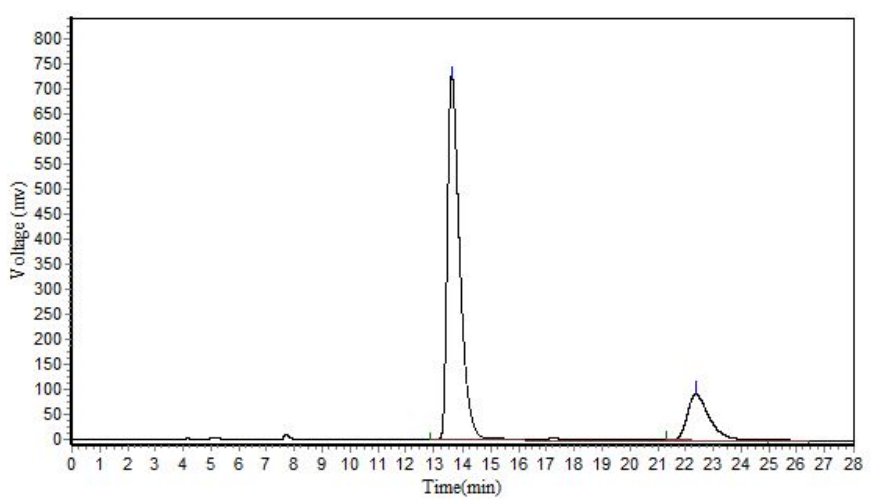

\begin{tabular}{|c|c|c|c|c|c|}
\hline \multicolumn{6}{|c|}{ Results } \\
\hline Peak No. & Peak ID & Ret Time & Height & Area & Conc. \\
\hline 1 & & 13.632 & 727834.813 & 22845452.000 & 81.9294 \\
\hline 2 & & 22.398 & 91078.742 & 5038873.500 & 18.0706 \\
\hline Total & & & 818913.555 & 27884325.500 & 100.0000 \\
\hline
\end{tabular}


(S)-2,3-bis(4-(trifluoromethyl)phenyl)-1H-inden-1-ol (3d)
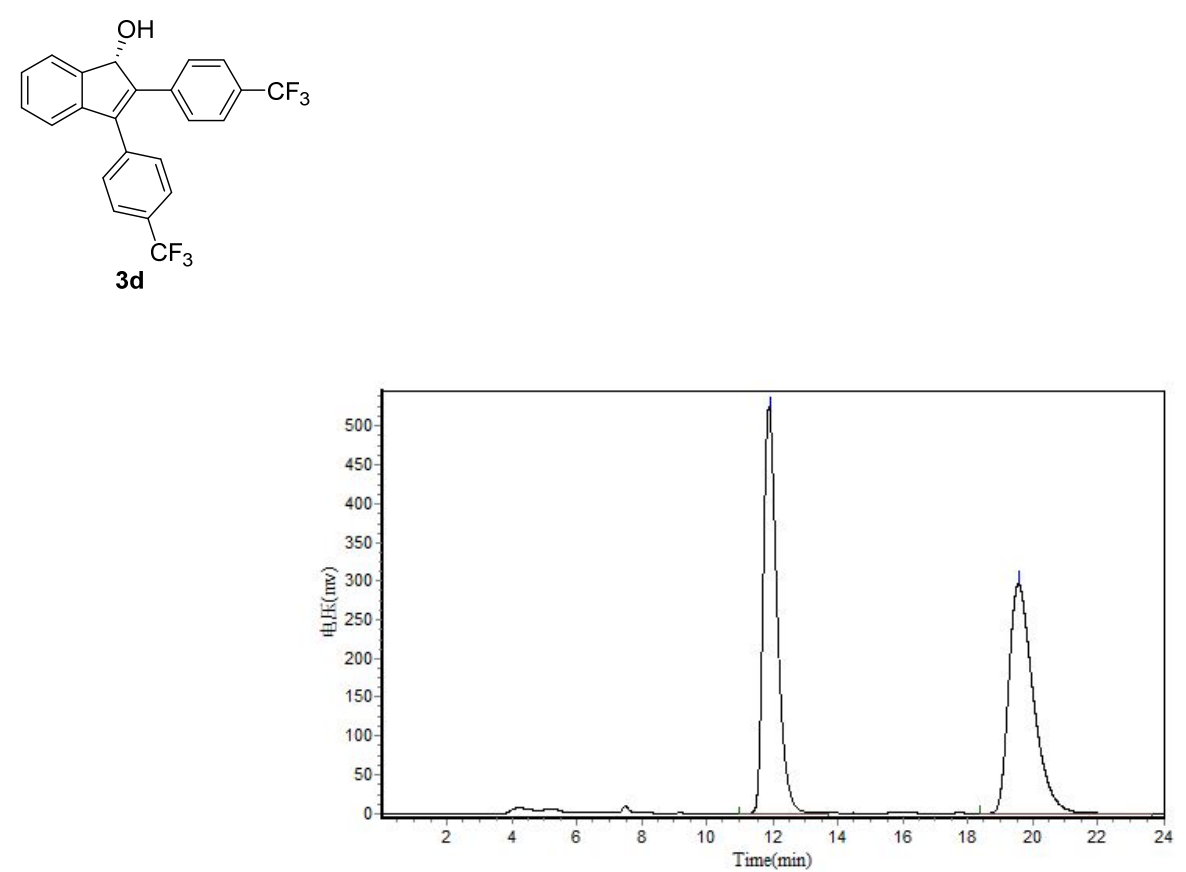

\begin{tabular}{cccccc} 
Peak No. & Peak ID & Ret Time & Height & Area & Conc. \\
\hline 1 & 11.898 & 523830.594 & 15611485.000 & 49.9907 \\
2 & 19.565 & 295931.906 & 15617323.000 & 50.0093 \\
\hline Total & & 819762.500 & 31228808.000 & 100.0000
\end{tabular}

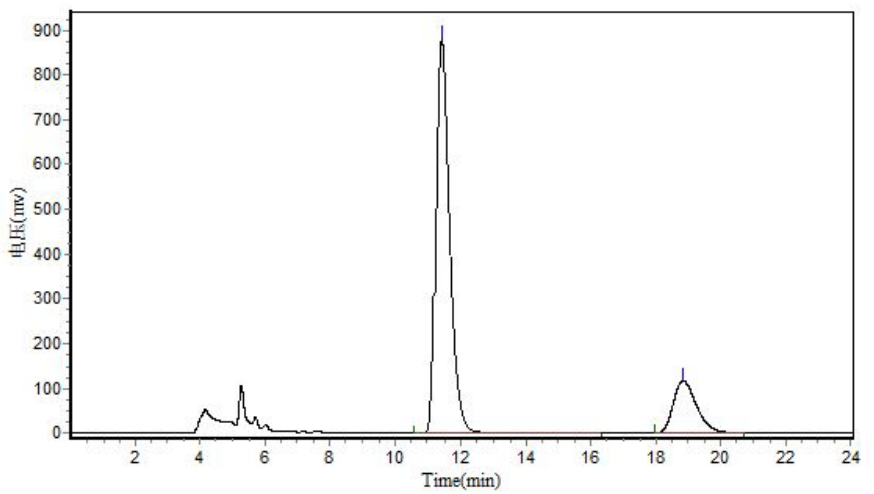

\begin{tabular}{|c|c|c|c|c|c|}
\hline \multicolumn{6}{|c|}{ Results } \\
\hline Peak No. & Peak ID & Ret Time & Height & Area & Conc. \\
\hline 1 & & 11.400 & 879039.125 & 24859256.000 & 81.0700 \\
\hline 2 & & 18.833 & 117045.938 & 5804673.000 & 18.9300 \\
\hline Total & & & 996085.063 & 30663929.000 & 100.0000 \\
\hline
\end{tabular}




\section{(S)-2,3-bis(4-fluorophenyl)-1H-inden-1-ol (3e)}
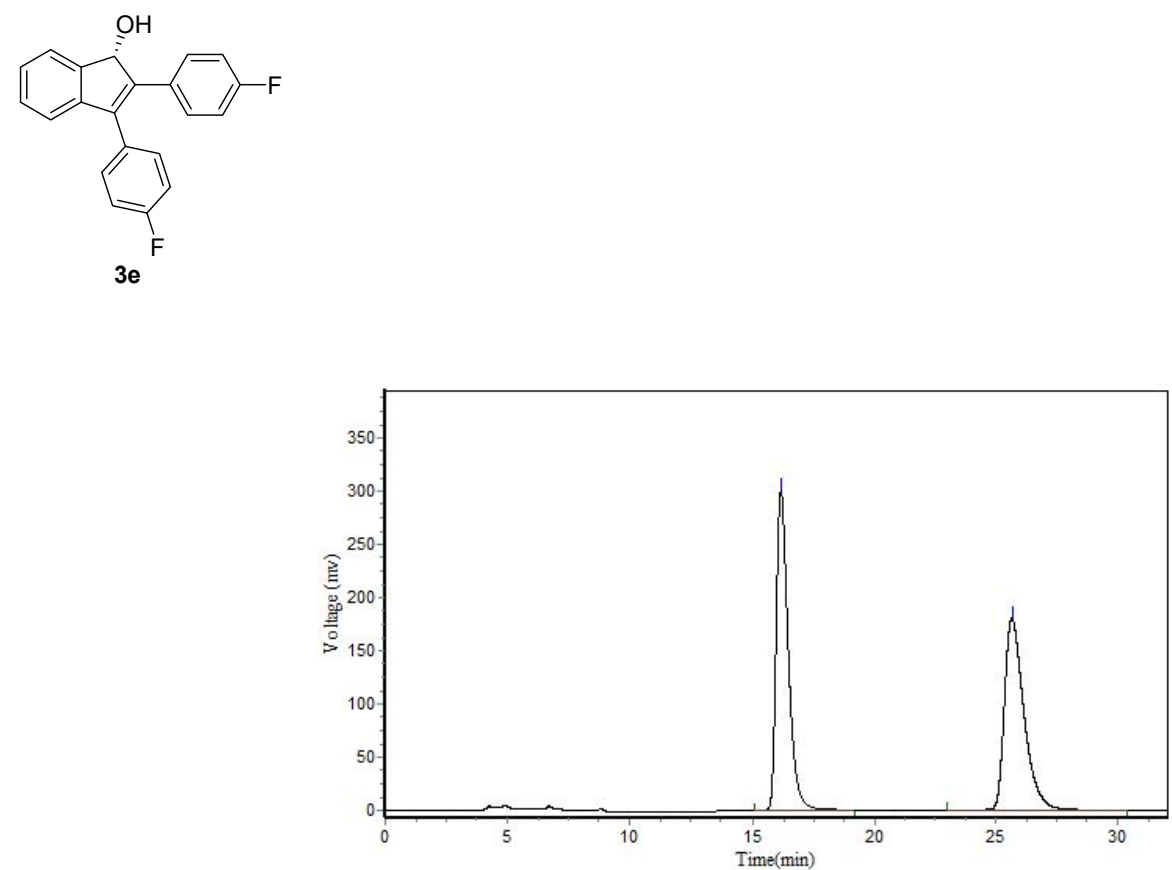

\begin{tabular}{|c|c|c|c|c|c|}
\hline \multicolumn{6}{|c|}{ Results } \\
\hline Peak No. & Peak ID & Ret Time & Height & Area & Conc. \\
\hline 1 & & 16.165 & 301616.375 & 10832460.000 & 50.4004 \\
\hline 2 & & 25.665 & 181115.203 & 10660346.000 & 49.5996 \\
\hline & & & 482731.578 & 21492806.000 & 100.000 \\
\hline
\end{tabular}

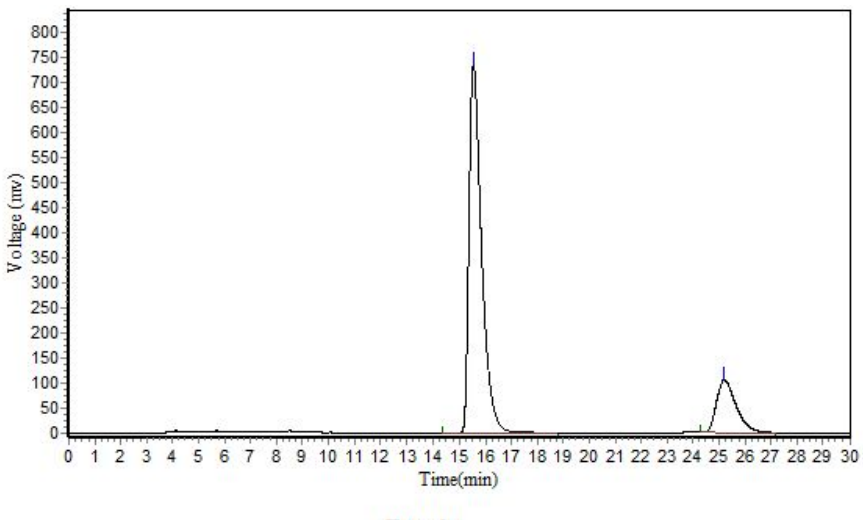

\begin{tabular}{|c|c|c|c|c|c|}
\hline \multicolumn{6}{|c|}{ Results } \\
\hline Peak No. & Peak ID & Ret Time & Height & Area & Conc. \\
\hline 1 & & 15.535 & 734200.750 & 24791864.000 & 81.7476 \\
\hline 2 & & 25.198 & 104815.445 & 5535460.500 & 18.2524 \\
\hline Total & & & 839016.195 & 30327324.500 & 100.0000 \\
\hline
\end{tabular}




\section{(S)-2,3-bis(3-methoxyphenyl)-1H-inden-1-ol (3f)}<smiles></smiles>

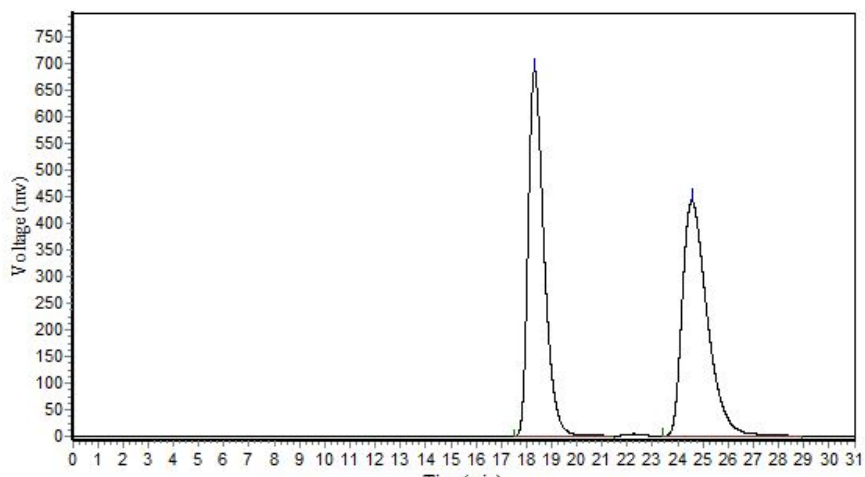

Results

\begin{tabular}{|c|c|c|c|c|c|}
\hline \multicolumn{6}{|c|}{ Results } \\
\hline Peak No. & Peak ID & Ret Time & Height & Area & Conc. \\
\hline 1 & & 18.332 & 687736.625 & 30174324.000 & 49.6581 \\
\hline 2 & & 24.598 & 442293.313 & 30589788.000 & 50.3419 \\
\hline Total & & & 1130029.938 & 60764112.000 & 100.0000 \\
\hline
\end{tabular}

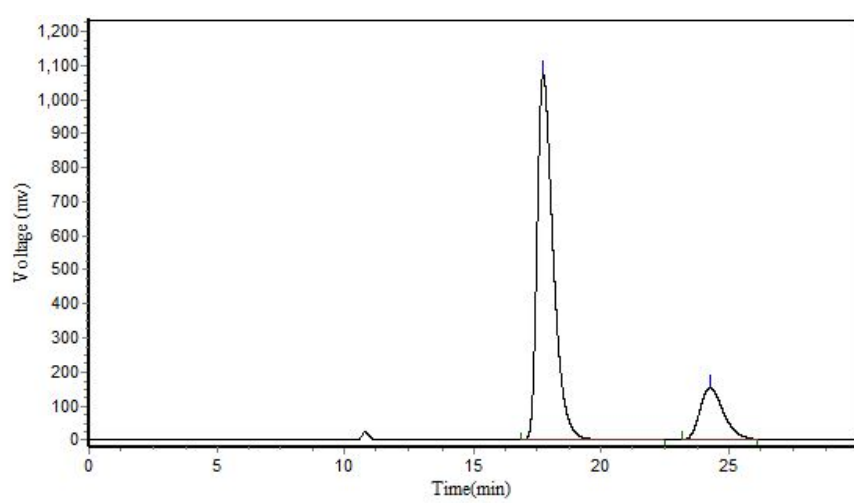

Results

\begin{tabular}{|c|c|c|c|c|c|}
\hline \multicolumn{6}{|c|}{ Acsuns } \\
\hline Peak No. & Peak ID & Ret Time & Height & Area & Conc. \\
\hline 1 & & 17.727 & 1075284.500 & 46898688.000 & 82.8129 \\
\hline 2 & & 24.260 & 151665.375 & 9733434.000 & 17.1871 \\
\hline
\end{tabular}




\section{(S)-2,3-di-m-tolyl-1H-inden-1-ol (3g)}
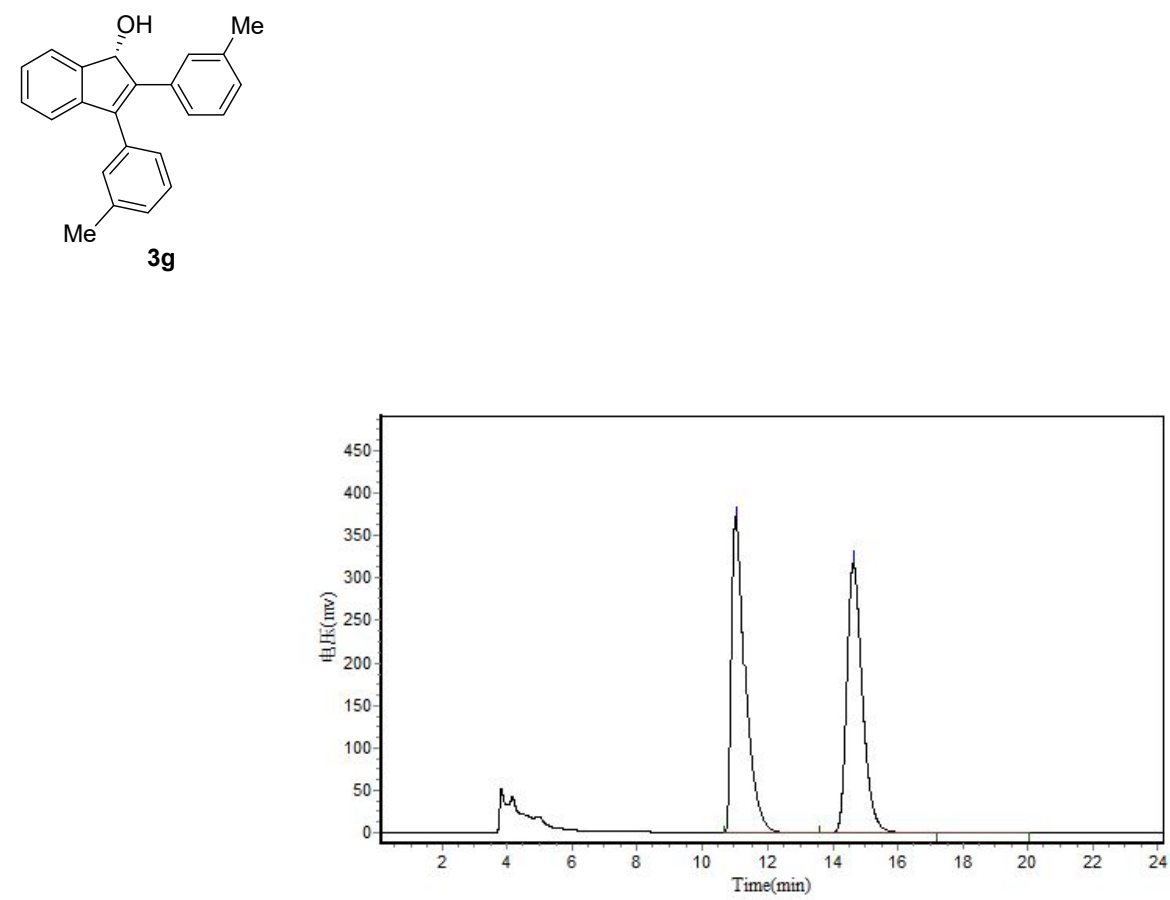

\begin{tabular}{cccccc} 
& \multicolumn{2}{c}{ Results } & Area & Conc. \\
\hline Peak No. & Peak ID & Ret Time & Height & 10777186.000 & 50.4333 \\
2 & 11.065 & 370773.594 & 10592002.000 & 49.5667 \\
\hline Total & 14.632 & 315868.063 & 686641.656 & 21369188.000 & 100.0000
\end{tabular}

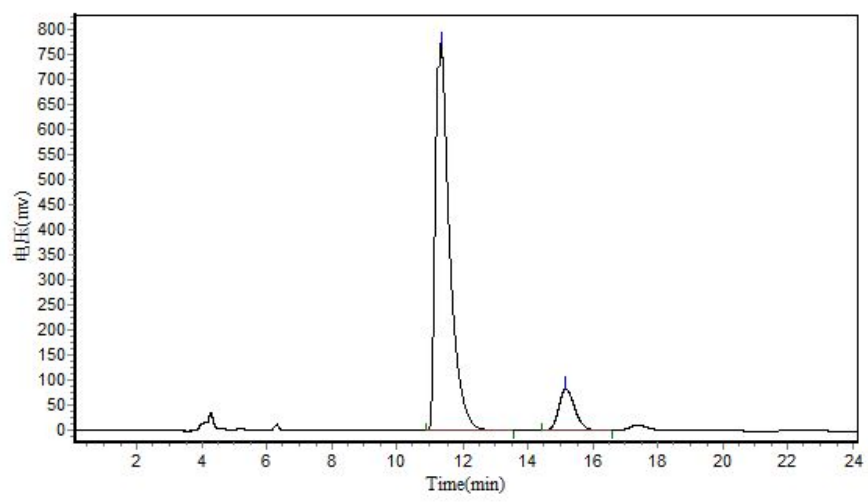

\begin{tabular}{cccccc} 
& \multicolumn{3}{c}{ Results } & Area & Conc. \\
\hline Peak No. & Peak ID & Ret Time & Height & 21794300.000 & 88.2124 \\
2 & 11.315 & 771690.188 & 2912321.500 & 11.7876 \\
\hline Total & 15.157 & 83610.219 & 24706621.500 & 100.0000
\end{tabular}




\section{(S)-2,3-bis(3-fluorophenyl)-1H-inden-1-ol (3h)}<smiles>OC1C(c2cccc(F)c2)=C(c2cccc(F)c2)c2ccccc21</smiles>

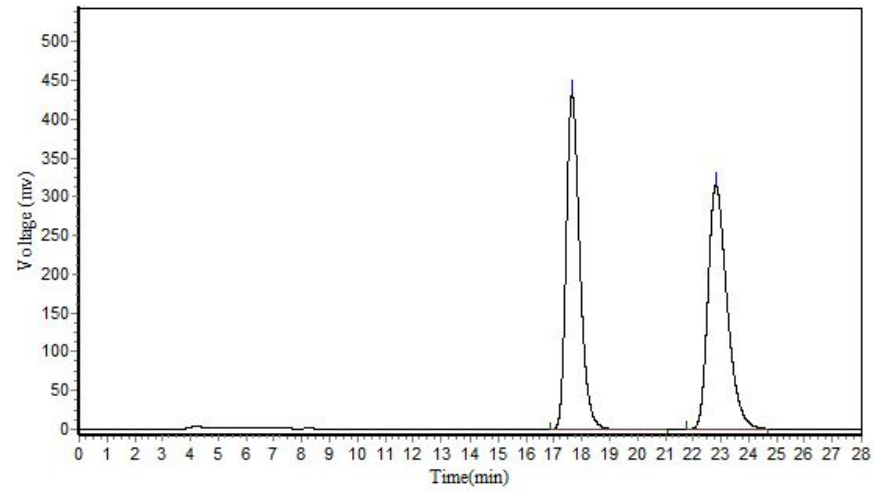

\begin{tabular}{|c|c|c|c|c|c|}
\hline \multicolumn{6}{|c|}{ Results } \\
\hline Peak No. & Peak ID & Ret Time & Height & Area & Conc. \\
\hline 1 & & 17.650 & 434300.438 & 14770106.000 & 49.6655 \\
\hline 2 & & 22.822 & 315993.000 & 14969089.000 & 50.3345 \\
\hline Total & & & 750293.438 & 29739195.000 & 100.0000 \\
\hline
\end{tabular}

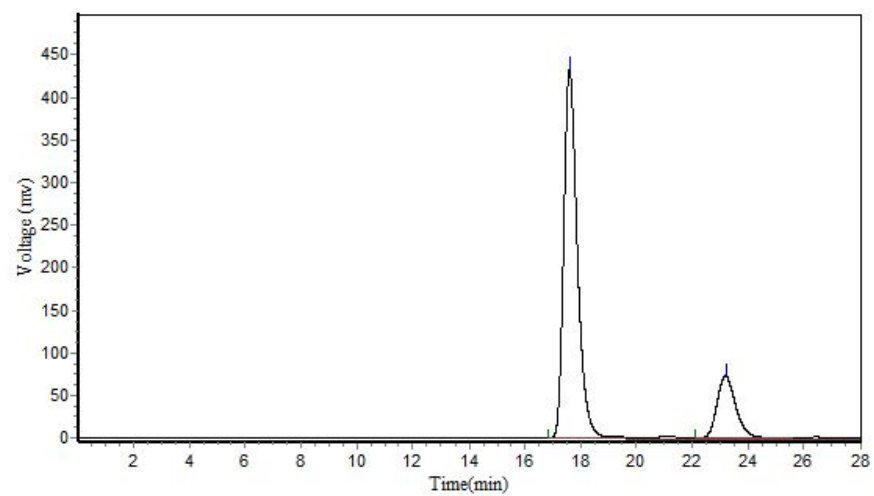

\begin{tabular}{|c|c|c|c|c|c|}
\hline \multicolumn{6}{|c|}{ Results } \\
\hline Peak No. & Peak ID & Ret Time & Height & Area & Conc. \\
\hline 1 & & 17.598 & 431436.594 & 15165834.000 & 81.3057 \\
\hline 2 & & 23.265 & 72812.094 & 3487029.750 & 18.6943 \\
\hline Total & & & 504248.688 & 18652863.750 & 100.0000 \\
\hline
\end{tabular}




\section{(S)-6-methoxy-2,3-diphenyl-1H-inden-1-ol (3i)}<smiles>COc1ccc2c(c1)[C@H](O)C(c1ccccc1)=C2c1ccccc1</smiles>

$3 i$
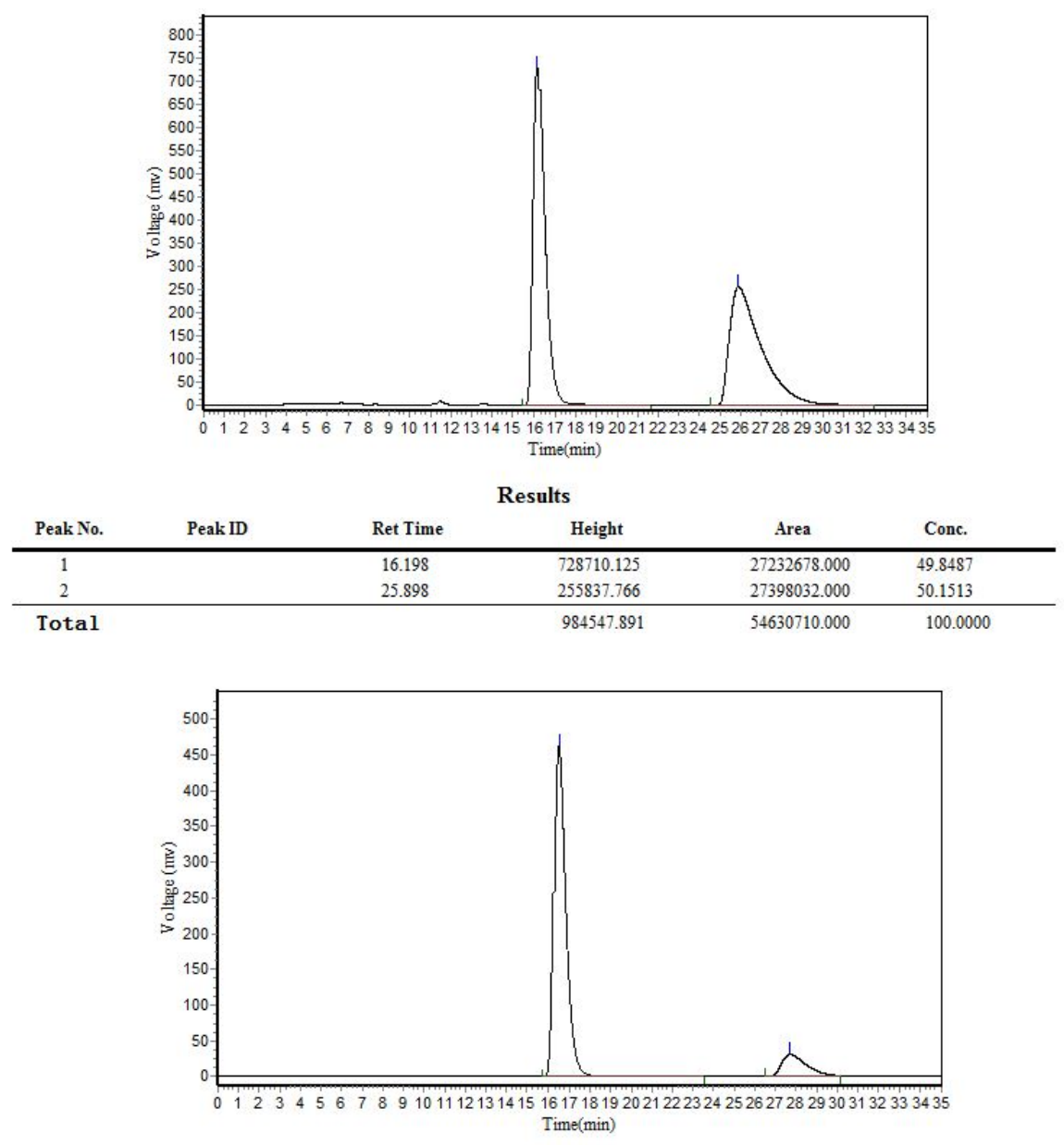

Results

\begin{tabular}{|c|c|c|c|c|c|}
\hline Peak No. & Peak ID & Ret Time & Height & Area & Conc. \\
\hline 1 & & 16.488 & 461775.875 & 17717384.000 & 87.5646 \\
\hline 2 & & 27.717 & 30284.725 & 2516114.500 & 12.4354 \\
\hline Total & & & 492060.600 & 20233498.500 & 100.0000 \\
\hline
\end{tabular}




\section{(S)-2,3-bis(4-fluorophenyl)-6-methoxy-1H-inden-1-ol (3j)}

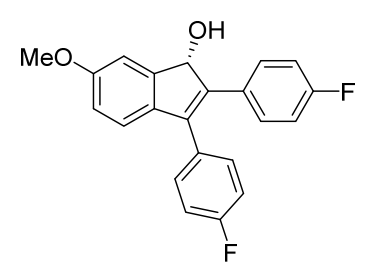

3j

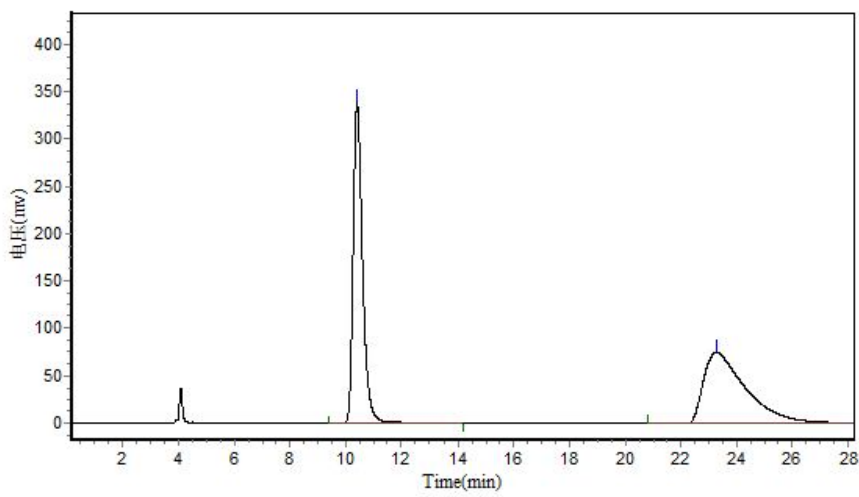

\begin{tabular}{|c|c|c|c|c|c|}
\hline \multicolumn{6}{|c|}{ Results } \\
\hline Peak No. & Peak ID & Ret Time & Height & Area & Conc. \\
\hline 1 & & 10.415 & 338010.094 & 7967826.500 & 50.2059 \\
\hline 2 & & 23.282 & 74895.734 & 7902467.000 & 49.7941 \\
\hline Total & & & 412905.828 & 15870293.500 & 100.0000 \\
\hline
\end{tabular}

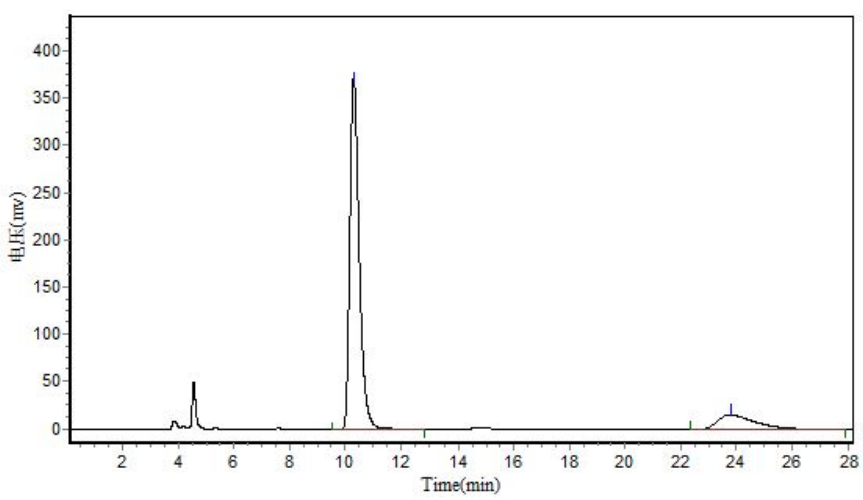

\begin{tabular}{|c|c|c|c|c|c|}
\hline \multicolumn{6}{|c|}{ Results } \\
\hline Peak No. & Peak ID & Ret Time & Height & Area & Conc. \\
\hline 1 & & 10.332 & 369887.906 & 8823576.000 & 84.4334 \\
\hline 2 & & 23.798 & 15802.972 & 1626756.000 & 15.5665 \\
\hline Total & & & 385690.878 & 10450332.000 & 100.0000 \\
\hline
\end{tabular}




\section{(S)-6-fluoro-2,3-diphenyl-1H-inden-1-ol (3k)}

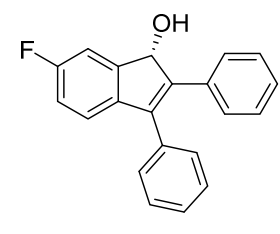

$3 k$

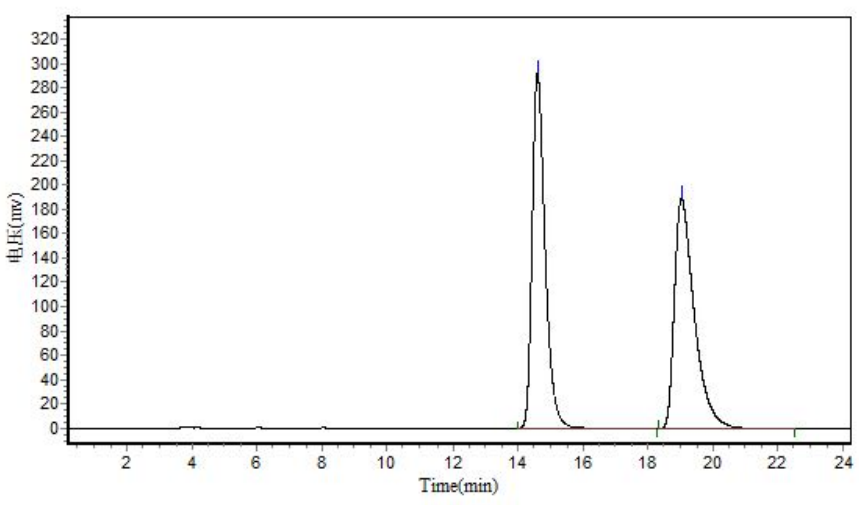

\begin{tabular}{|c|c|c|c|c|c|}
\hline \multicolumn{6}{|c|}{ Results } \\
\hline Peak No. & Peak ID & Ret Time & Height & Area & Conc. \\
\hline$\overline{1}$ & & 14.615 & 291478.688 & 8184336.500 & 50.2975 \\
\hline 2 & & 19.048 & 188872.000 & 8087524.000 & 49.7025 \\
\hline Total & & & 480350.688 & 16271860.500 & 100.0000 \\
\hline
\end{tabular}

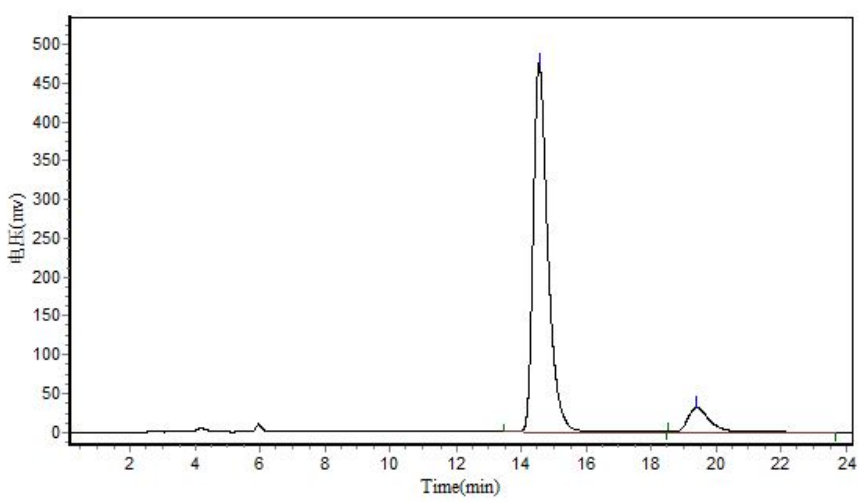

\begin{tabular}{|c|c|c|c|c|c|}
\hline \multicolumn{6}{|c|}{ Results } \\
\hline Peak No. & Peak ID & Ret Time & Height & Area & Conc. \\
\hline 1 & & 14.565 & 474702.094 & 14891386.000 & 91.0232 \\
\hline 2 & & 19.398 & 31178.830 & 1468603.000 & 8.9768 \\
\hline Total & & & 505880.924 & 16359989.000 & 100.0000 \\
\hline
\end{tabular}


(S)-6-fluoro-2,3-bis(4-methoxyphenyl)-1H-inden-1-ol (3I)

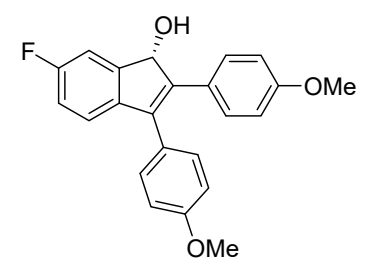

31

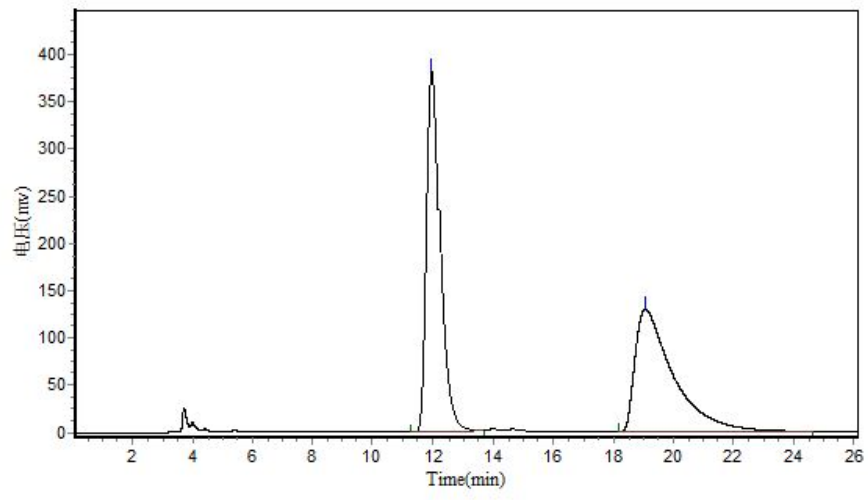

\begin{tabular}{|c|c|c|c|c|c|}
\hline \multicolumn{6}{|c|}{ Results } \\
\hline Peak No. & Peak ID & Ret Time & Height & Area & Conc. \\
\hline 1 & & 11.982 & 379972.781 & 11542180.000 & 50.1761 \\
\hline 2 & & 19.063 & 129624.813 & 11461171.000 & 49.8239 \\
\hline Total & & & 509597.594 & 23003351.000 & 100.0000 \\
\hline
\end{tabular}

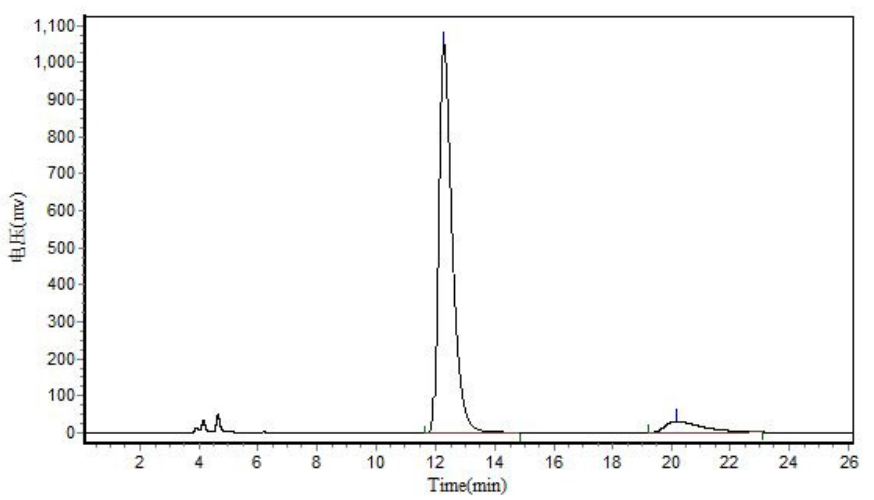

\begin{tabular}{|c|c|c|c|c|c|}
\hline \multicolumn{6}{|c|}{ Results } \\
\hline Peak No. & Peak ID & Ret Time & Height & Area & Conc. \\
\hline 1 & & 12.268 & 1047614.313 & 32917426.000 & 92.8310 \\
\hline 2 & & 20.195 & 29474.201 & 2542109.000 & 7.1690 \\
\hline Total & & & 1077088.514 & 35459535.000 & 100.0000 \\
\hline
\end{tabular}




\section{(S)-6-fluoro-2,3-di-p-tolyl-1H-inden-1-ol (3m)}

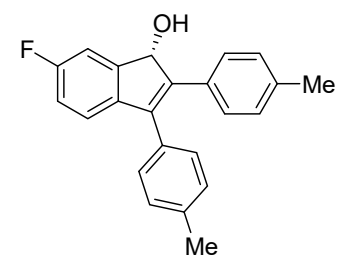

$3 m$

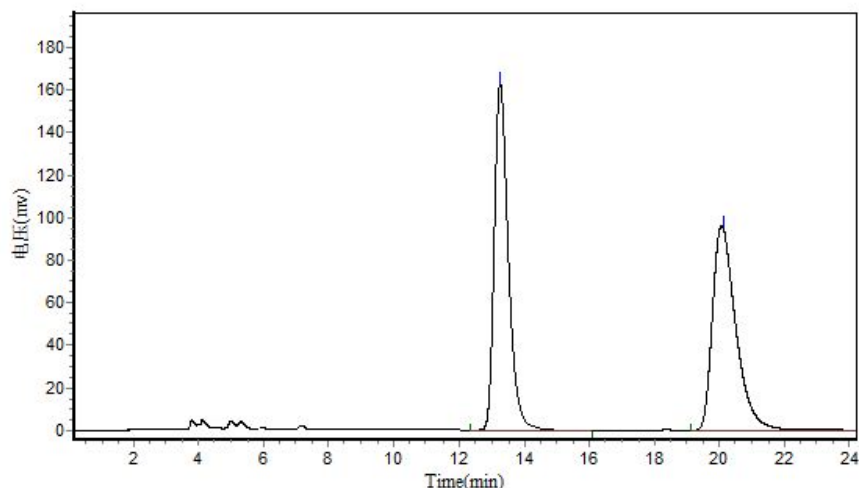

\begin{tabular}{|c|c|c|c|c|c|}
\hline \multicolumn{6}{|c|}{ Results } \\
\hline Peak No. & Peak ID & Ret Time & Height & Area & Conc. \\
\hline 1 & & 13.265 & 162591.781 & 4912361.000 & 50.2574 \\
\hline 2 & & 20.132 & 95931.516 & 4862047.000 & 49.7426 \\
\hline Total & & & 258523.297 & 9774408.000 & 100.0000 \\
\hline
\end{tabular}

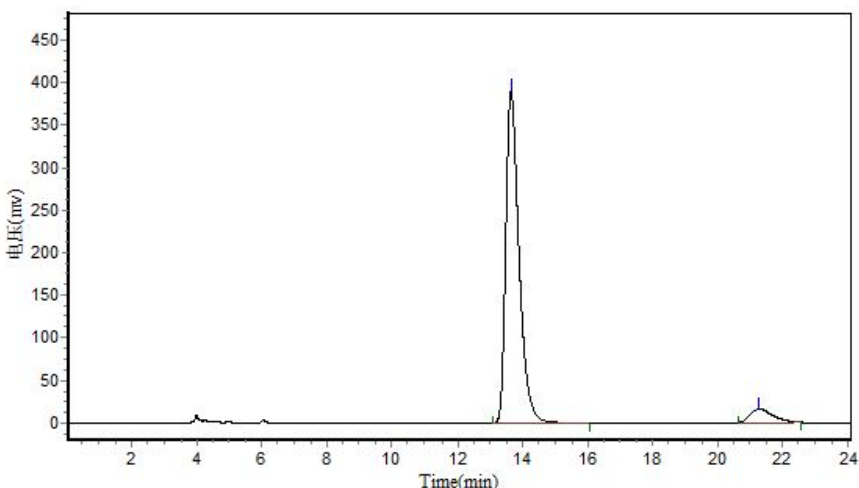

\begin{tabular}{|c|c|c|c|c|c|}
\hline \multicolumn{6}{|c|}{ Results } \\
\hline Peak No. & Peak ID & Ret Time & Height & Area & Conc. \\
\hline 1 & & 13.640 & 389453.906 & 11066257.000 & 93.4311 \\
\hline 2 & & 21.263 & 15925.048 & 778034.813 & 6.5689 \\
\hline Total & & & 405378.954 & 11844291.813 & 100.0000 \\
\hline
\end{tabular}




\section{(S)-6-fluoro-2,3-bis(4-(trifluoromethyl)phenyl)-1H-inden-1-ol (3n)}

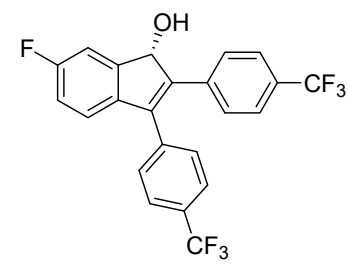

$3 n$

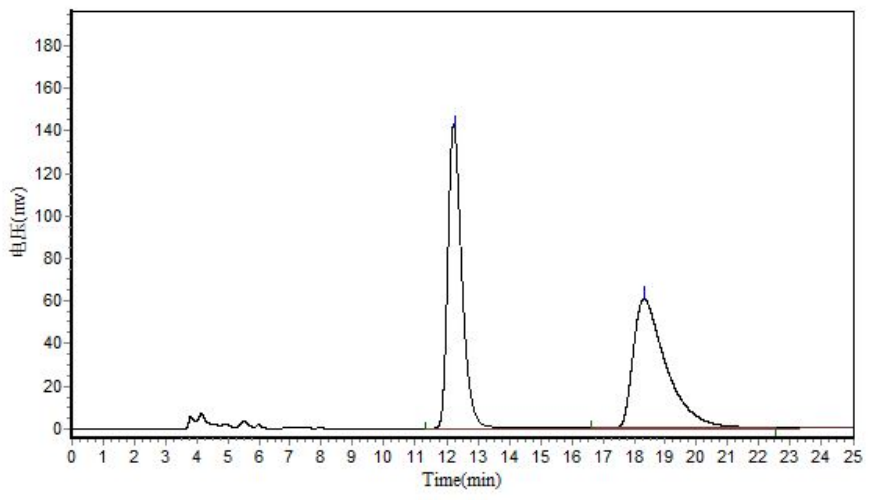

\begin{tabular}{|c|c|c|c|c|c|}
\hline \multicolumn{6}{|c|}{ Results } \\
\hline Peak No. & Peak ID & Ret Time & Height & Area & Conc. \\
\hline 1 & & 12.232 & 142212.578 & 4507281.500 & 50.0553 \\
\hline 2 & & 18.332 & 60610.355 & 4497322.000 & 49.9447 \\
\hline Total & & & 202822.934 & 9004603.500 & 100.0000 \\
\hline
\end{tabular}

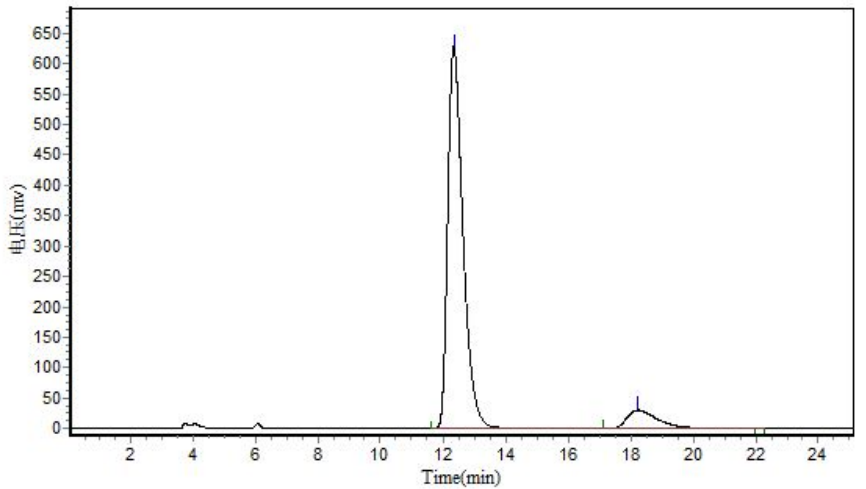

\begin{tabular}{|c|c|c|c|c|c|}
\hline \multicolumn{6}{|c|}{ Results } \\
\hline Peak No. & Peak ID & Ret Time & Height & Area & Conc. \\
\hline 1 & & 12.332 & 627719.750 & 21546890.000 & 91.0165 \\
\hline 2 & & 18.232 & 30266.939 & 2126711.250 & 8.9835 \\
\hline Total & & & 657986.689 & 23673601.250 & 100.0000 \\
\hline
\end{tabular}




\section{(S)-6-fluoro-2,3-bis(4-fluorophenyl)-1H-inden-1-ol (3o)}

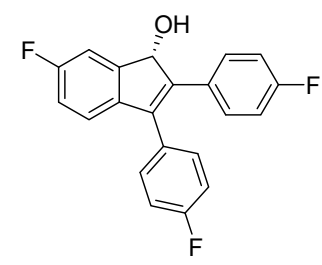

30

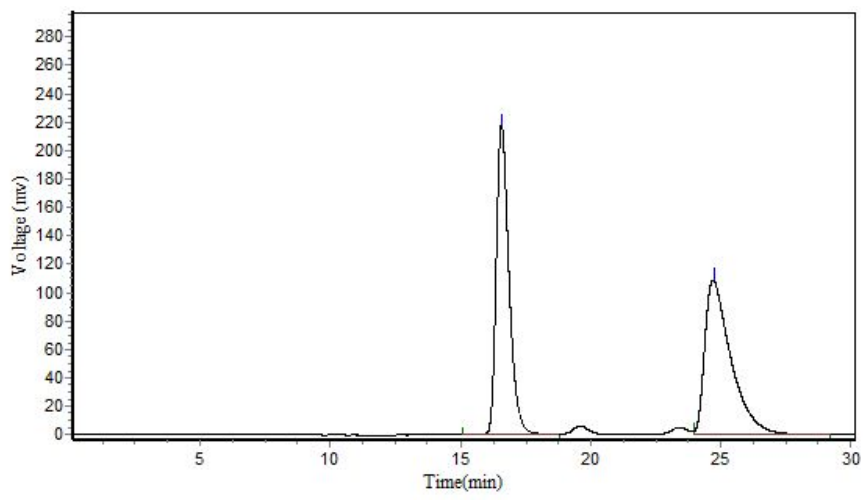

Results

\begin{tabular}{|c|c|c|c|c|c|}
\hline Peak No. & Peak ID & Ret Time & Height & Area & Conc. \\
\hline 1 & & 16.565 & 218047.375 & 7527600.500 & 49.6527 \\
\hline 2 & & 24.732 & 108793.586 & 7632891.000 & 50.3473 \\
\hline Total & & & 326840.961 & 15160491.500 & 100.0000 \\
\hline
\end{tabular}

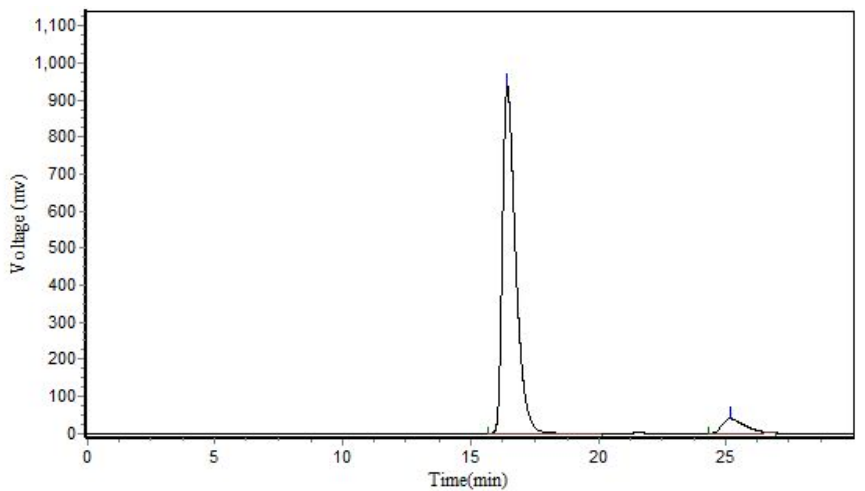

Results

\begin{tabular}{|c|c|c|c|c|c|}
\hline Peak No. & Peak ID & Ret Time & Height & Area & Conc. \\
\hline 1 & & 16.422 & 937878.125 & 33658340.000 & 93.1263 \\
\hline 2 & & 25.155 & 39479.609 & 2484340.750 & 6.8737 \\
\hline Total & & & 977357.734 & 36142680.750 & 100.0000 \\
\hline
\end{tabular}




\section{(S)-methyl-1-hydroxy-3-methyl-1H-indene-2-carboxylate (5a)}<smiles>CC(=O)C1=C(C)c2ccccc2[C@H]1O</smiles>

$5 a$

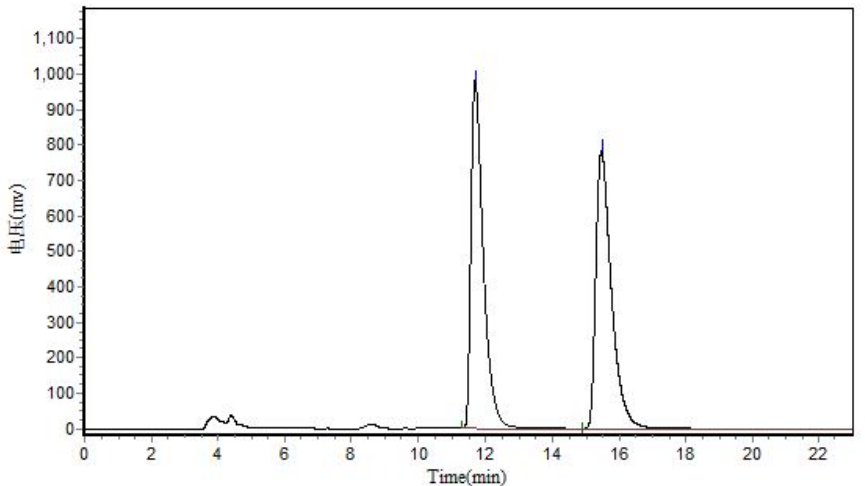

\begin{tabular}{|c|c|c|c|c|c|}
\hline \multicolumn{6}{|c|}{ Results } \\
\hline Peak No. & Peak ID & Ret Time & Height & Area & Conc. \\
\hline 1 & & 11.698 & 979877.875 & 25606158.000 & 49.9278 \\
\hline 2 & & 15.498 & 782753.188 & 25680170.000 & 50.0722 \\
\hline Total & & & 1762631.063 & 51286328.000 & 100.0000 \\
\hline
\end{tabular}

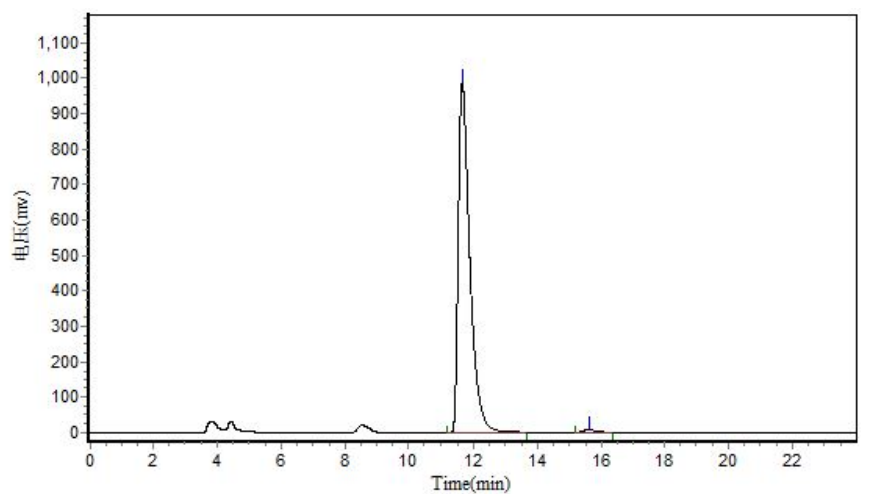

\begin{tabular}{|c|c|c|c|c|c|}
\hline \multicolumn{6}{|c|}{ Results } \\
\hline Peak No. & Peak ID & Ret Time & Height & Area & Conc. \\
\hline 1 & & 11.635 & 986929.000 & 24876256.000 & 99.1458 \\
\hline 2 & & 15.613 & 7673.041 & 214312.703 & 0.8542 \\
\hline Total & & & 994602.041 & 25090568.703 & 100.0000 \\
\hline
\end{tabular}




\section{(R)-methyl -1-hydroxy-2-methyl-1H-indene-3-carboxylate (6a)}

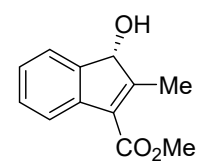

$6 a$

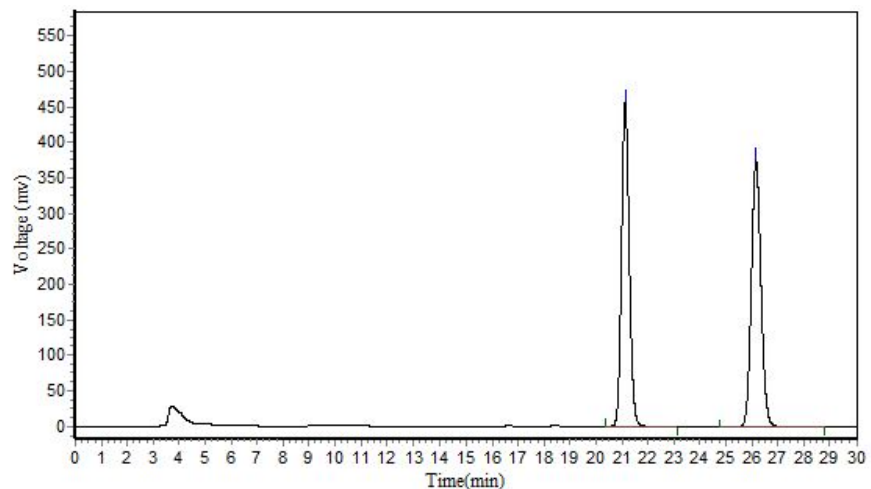

Results

\begin{tabular}{|c|c|c|c|c|c|}
\hline \multicolumn{6}{|c|}{ 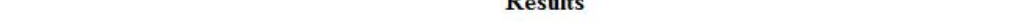 } \\
\hline Peak No. & Peak ID & Ret Time & Height & Area & Conc. \\
\hline 1 & & 21.120 & 455474.625 & 9436841.000 & 49.4876 \\
\hline 2 & & 26.158 & 373464.688 & 9632245.000 & 50.5124 \\
\hline Total & & & 828939.313 & 19069086.000 & 100.0000 \\
\hline
\end{tabular}

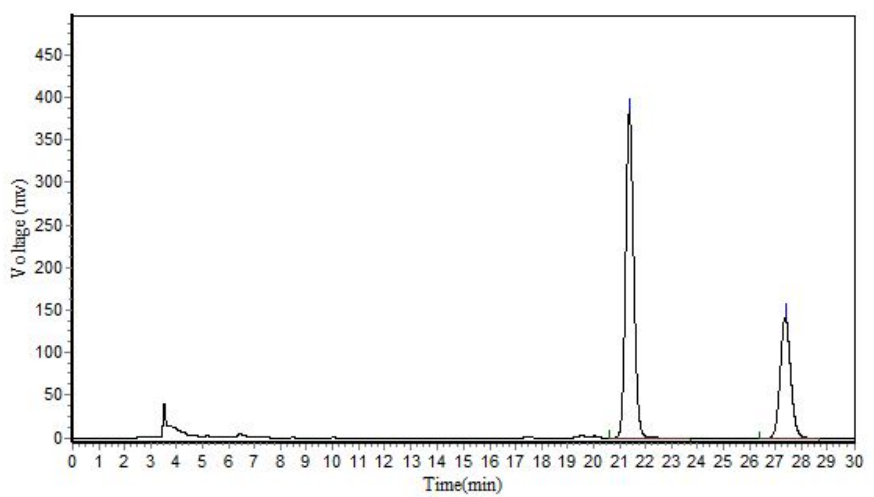

Results

\begin{tabular}{|c|c|c|c|c|c|}
\hline Peak No. & Peak ID & Ret Time & Height & Area & Conc. \\
\hline 1 & & 21.382 & 383402.563 & 8298215.000 & 67.5508 \\
\hline 2 & & 27.382 & 143626.375 & 3986201.500 & 32.4493 \\
\hline Total & & & 527028.938 & 12284416.500 & 100.0000 \\
\hline
\end{tabular}




\section{(S)-methyl -1-hydroxy-3-propyl-1H-indene-2-carboxylate (5b)}

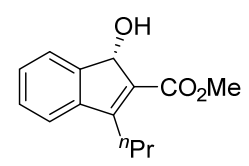

$5 b$

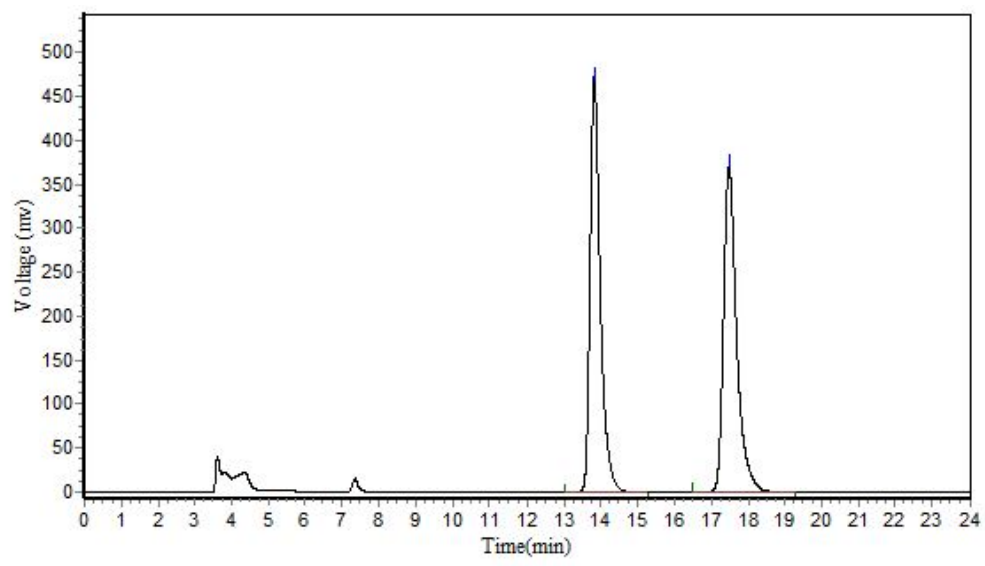

Results

\begin{tabular}{cccccc} 
Peak No. & Peak ID & Ret Time & Height & Area & Conc. \\
\hline 1 & & 13.832 & 470838.938 & 9118028.000 & 50.0135 \\
2 & 17.498 & 369516.813 & 9113087.000 & 49.9864 \\
\hline Total & & 840355.750 & 18231115.000 & 100.0000
\end{tabular}

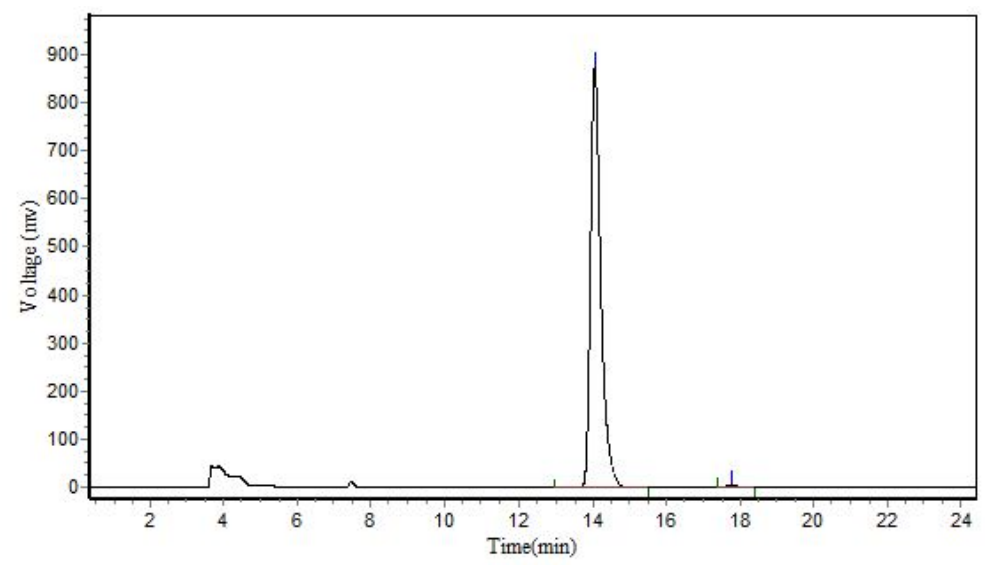

Results

\begin{tabular}{|c|c|c|c|c|c|}
\hline Peak No. & Peak ID & Ret Time & Height & Area & Conc. \\
\hline 1 & & 14.053 & 871827.063 & 17017080.000 & 99.2234 \\
\hline 2 & & 17.757 & 5363.034 & 133196.094 & 0.7766 \\
\hline Total & & & 877190.096 & 17150276.094 & 100.000 \\
\hline
\end{tabular}




\section{(R)-methyl -1-hydroxy-2-propyl-1H-indene-3-carboxylate (6b)}

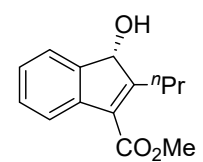

6b

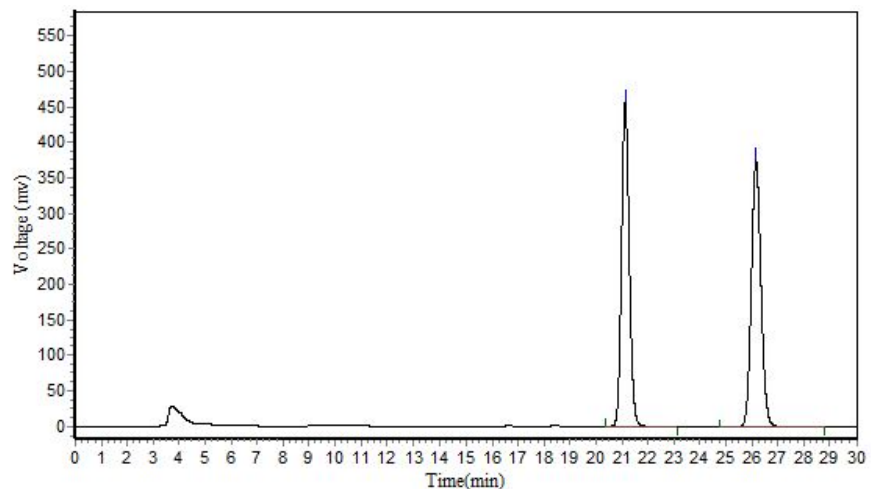

Results

\begin{tabular}{|c|c|c|c|c|c|}
\hline \multicolumn{6}{|c|}{ Kesuits } \\
\hline Peak No. & Peak ID & Ret Time & Height & Area & Conc. \\
\hline 1 & & 21.120 & 455474.625 & 9436841.000 & 49.4876 \\
\hline 2 & & 26.158 & 373464.688 & 9632245.000 & 50.5124 \\
\hline Total & & & 828939.313 & 19069086.000 & 100.0000 \\
\hline
\end{tabular}

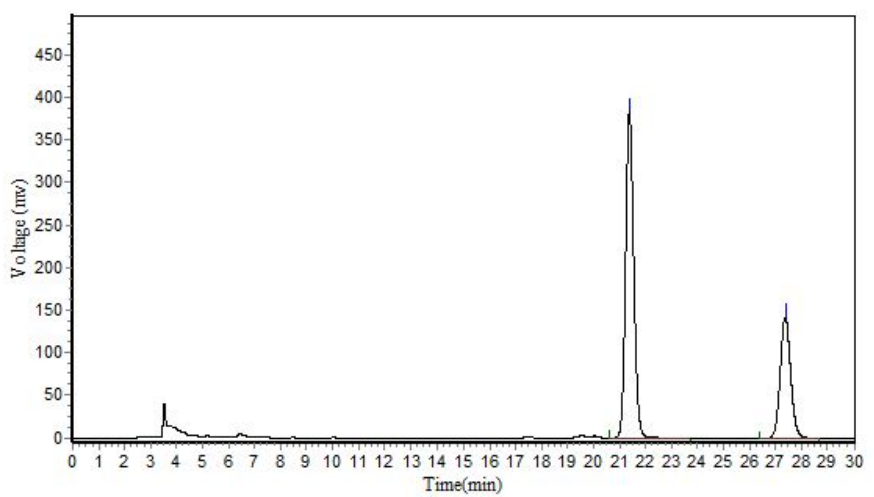

Results

\begin{tabular}{|c|c|c|c|c|c|}
\hline Peak No. & Peak ID & Ret Time & Height & Area & Conc. \\
\hline 1 & & 21.382 & 383402.563 & 8298215.000 & 67.5508 \\
\hline 2 & & 27.382 & 143626.375 & 3986201.500 & 32.4493 \\
\hline Total & & & 527028.938 & 12284416.500 & 100.0000 \\
\hline
\end{tabular}


(S)-methyl -1-hydroxy-3-phenyl-1H-indene-2-carboxylate (5c)

$\mathrm{CO}_{2} \mathrm{Me}$

$5 \mathrm{c}$

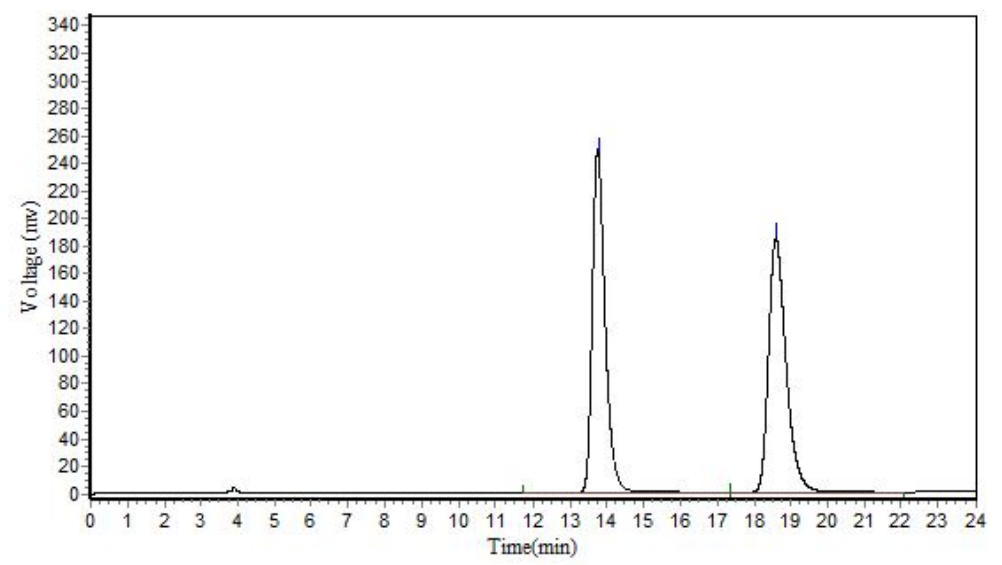

Results

\begin{tabular}{|c|c|c|c|c|c|}
\hline \multicolumn{6}{|c|}{ 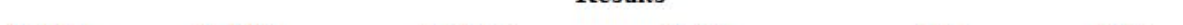 } \\
\hline Peak No. & Peak ID & Ret Time & Height & Area & Conc. \\
\hline 1 & & 13.765 & 248802.234 & 6091511.500 & 49.9686 \\
\hline 2 & & 18.598 & 184147.641 & 6099173.500 & 50.0314 \\
\hline Total & & & 432949.875 & 12190685.000 & 100.0000 \\
\hline
\end{tabular}

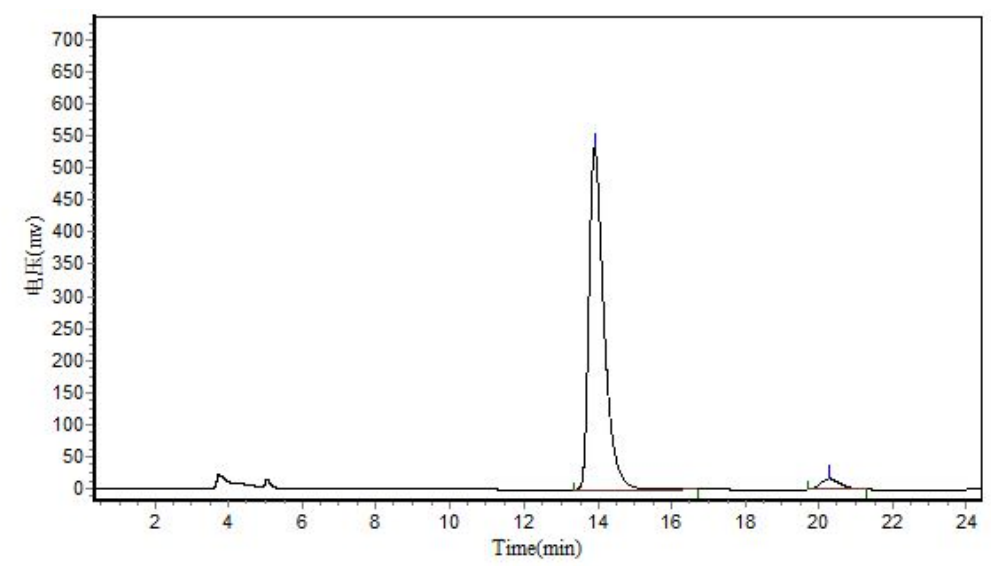

Results

\begin{tabular}{|c|c|c|c|c|c|}
\hline Peak No. & Peak ID & Ret Time & Height & Area & Conc. \\
\hline 1 & & 13.908 & 530871.875 & 15075456.000 & 95.9972 \\
\hline 2 & & 20.280 & 16666.836 & 628598.813 & 4.0028 \\
\hline Total & & & 547538.711 & 15704054.813 & 100.0000 \\
\hline
\end{tabular}


(S)-methyl -1-hydroxy-2-phenyl-1H-indene-3-carboxylate (6c)

Pro

$6 c$

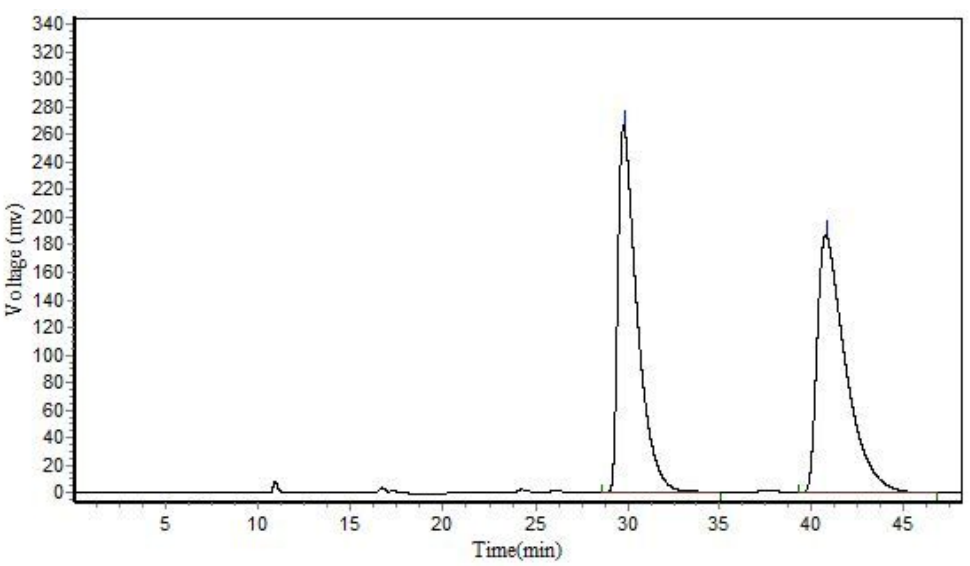

Results

\begin{tabular}{|c|c|c|c|c|c|}
\hline Peak No. & Peak ID & Ret Time & Height & Area & Conc. \\
\hline 1 & & 29.807 & 267316.719 & 19576752.000 & 49.1373 \\
\hline 2 & & 40.807 & 187443.906 & 20264194.000 & 50.8627 \\
\hline Total & & & 454760.625 & 39840946.000 & 100.0000 \\
\hline
\end{tabular}

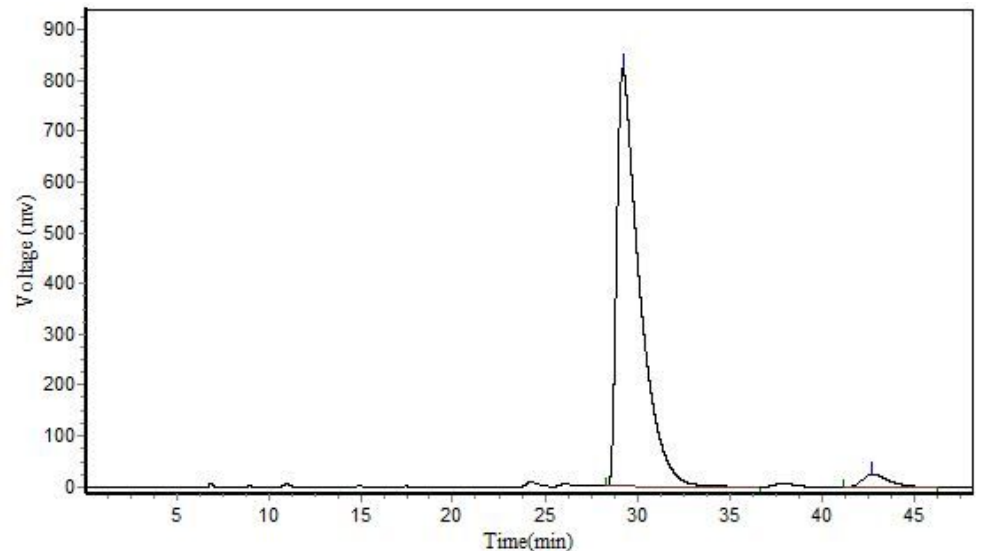

Results

\begin{tabular}{|c|c|c|c|c|c|}
\hline Peak No. & Peak ID & Ret Time & Height & Area & Conc. \\
\hline 1 & & 29.198 & 823478.688 & 70829728.000 & 96.3745 \\
\hline 2 & & 42.732 & 25467.621 & 2664497.250 & 3.6255 \\
\hline Total & & & 848946.309 & 73494225.250 & 100.000 \\
\hline
\end{tabular}


(S)-methyl -1-hydroxy-3-(4-methoxyphenyl)-1H-indene-2-carboxylate (5d)
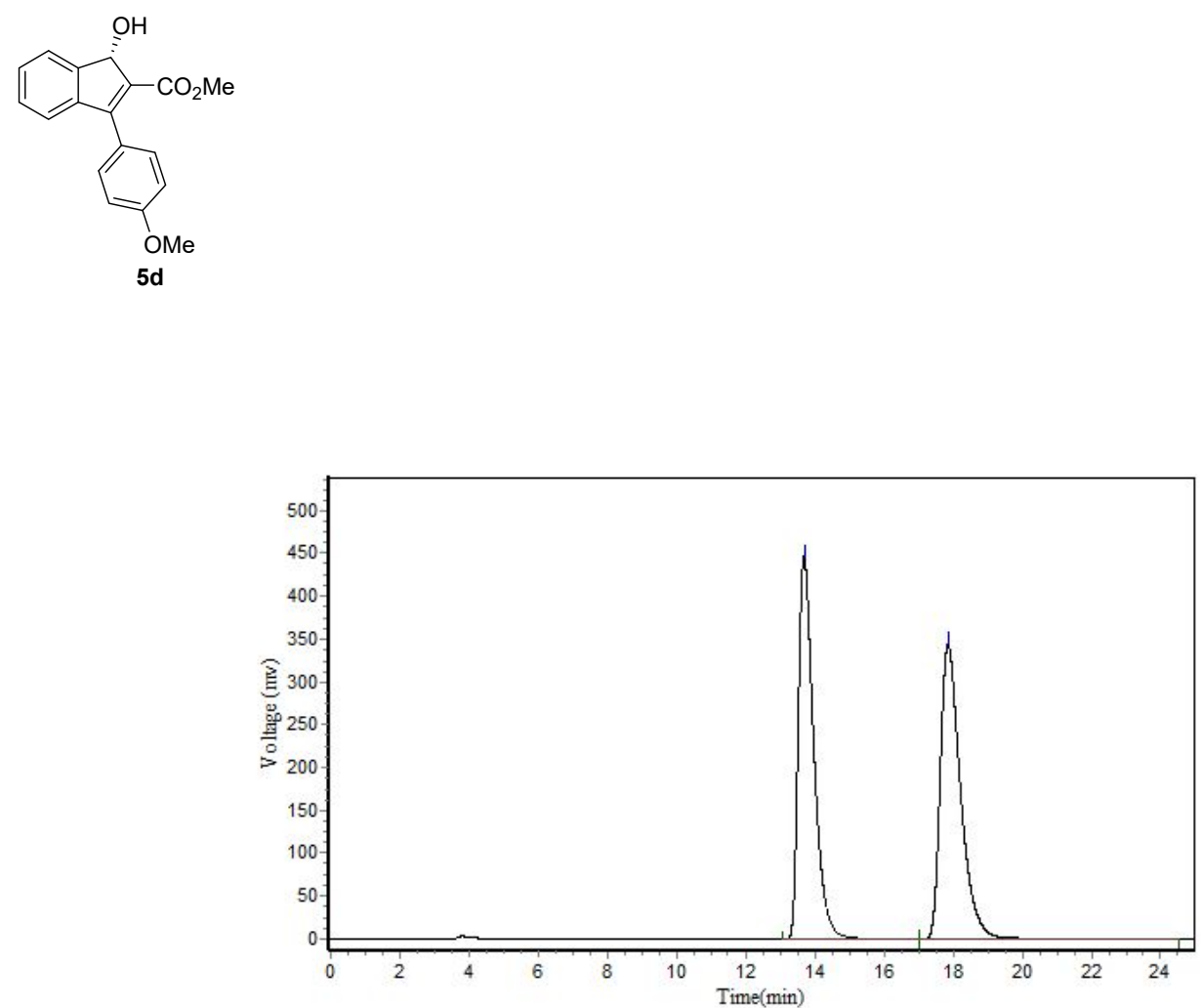

\begin{tabular}{|c|c|c|c|c|c|}
\hline \multicolumn{6}{|c|}{ Results } \\
\hline Peak No. & Peak ID & Ret Time & Height & Area & Conc. \\
\hline 1 & & 13.682 & 445234.313 & 13992830.000 & 49.7948 \\
\hline 2 & & 17.848 & 343235.594 & 14108140.000 & 50.2052 \\
\hline Total & & & 788469.906 & 28100970.000 & 100.0000 \\
\hline
\end{tabular}

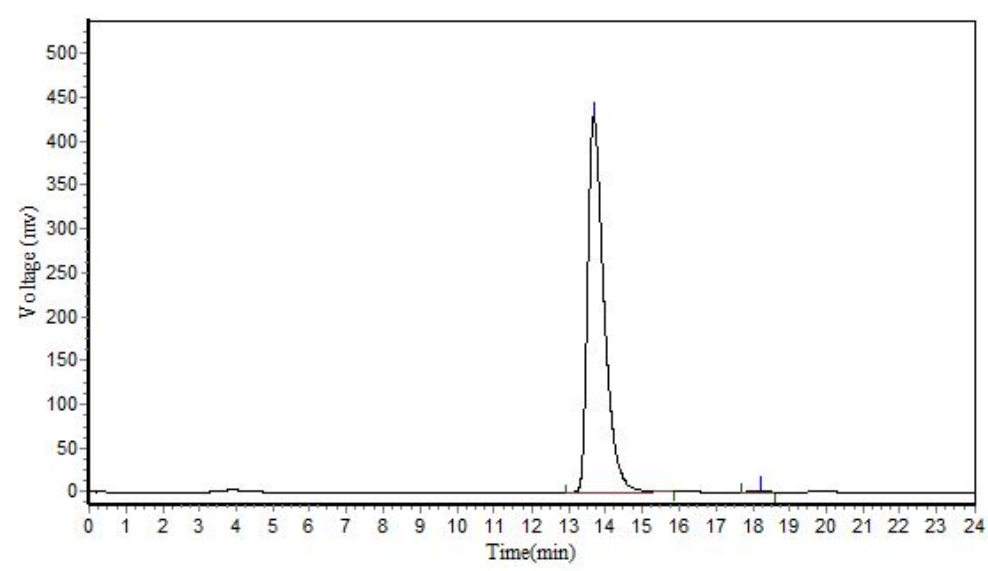

Results

\begin{tabular}{|c|c|c|c|c|c|}
\hline Peak No. & Peak ID & Ret Time & Height & Area & Conc. \\
\hline 1 & & 13.675 & 428132.031 & 13258955.000 & 99.9016 \\
\hline 2 & & 18.208 & 432.937 & 13056.350 & 0.0984 \\
\hline Total & & & 428564.969 & 13272011.350 & 100.000 \\
\hline
\end{tabular}




\section{(S)-methyl -6-fluoro-1-hydroxy-3-methyl-1H-indene-2-carboxylate (5e)}<smiles>CC(=O)OC1=C(C)c2ccc(F)cc2[C@H]1O</smiles>

$5 e$

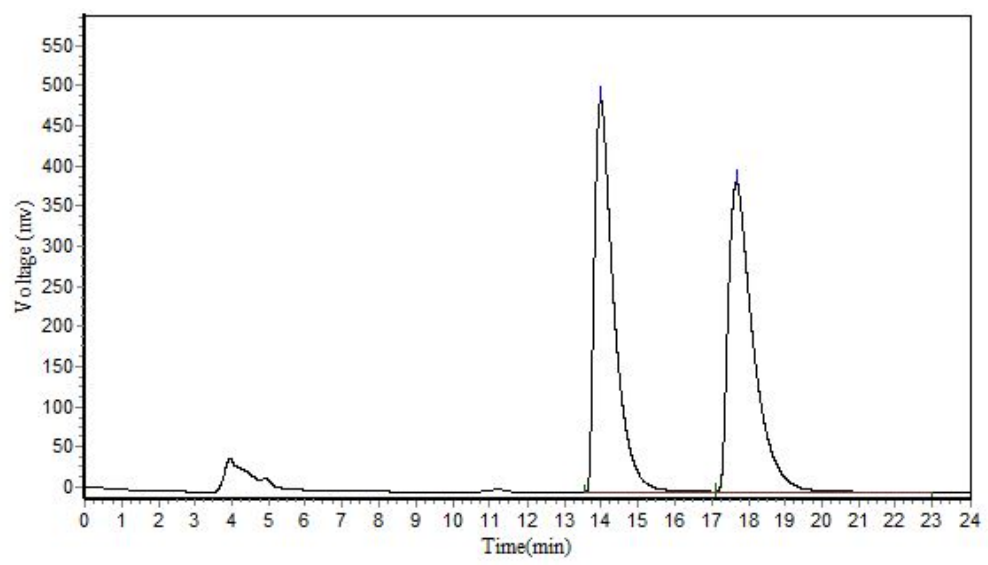

Results

\begin{tabular}{|c|c|c|c|c|c|}
\hline Peak No. & Peak ID & Ret Time & Height & Area & Conc. \\
\hline 1 & & 13.998 & 487874.156 & 17969076.000 & 49.6754 \\
\hline 2 & & 17.698 & 385564.531 & 18203918.000 & 50.3246 \\
\hline Total & & & 873438.688 & 36172994.000 & 100.0000 \\
\hline
\end{tabular}

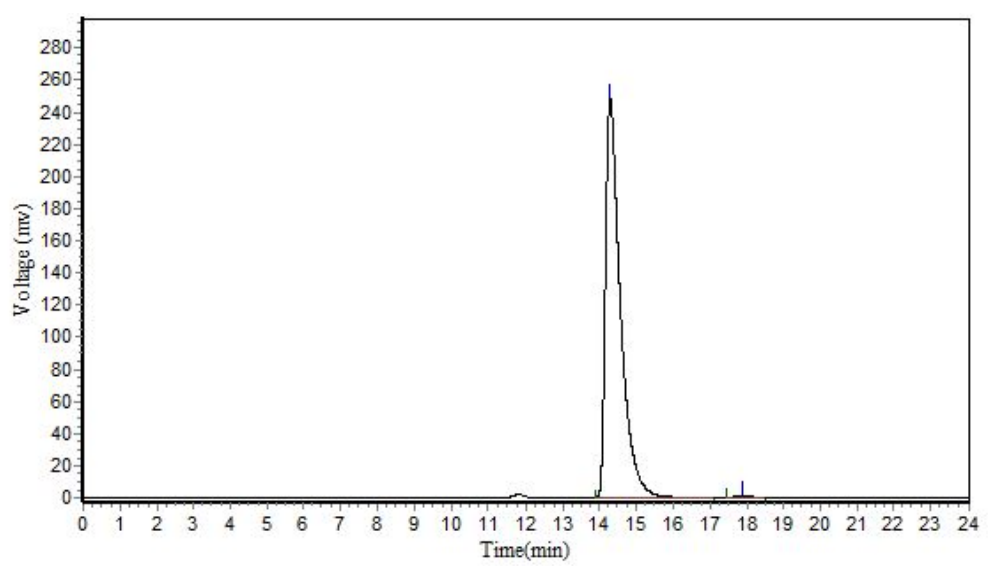

Results

\begin{tabular}{|c|c|c|c|c|c|}
\hline Peak No. & Peak ID & Ret Time & Height & Area & Conc. \\
\hline 1 & & 14.285 & 248011.969 & 7044342.000 & 99.4718 \\
\hline 2 & & 17.882 & 1258.828 & 37402.301 & 0.5282 \\
\hline Total & & & 249270.797 & 7081744.301 & 100.0000 \\
\hline
\end{tabular}


(R)-methyl -6-fluoro-1-hydroxy-2-methyl-1H-indene-3-carboxylate (6e)<smiles>COC(=O)C1=C(C)[C@H](O)c2cc(F)ccc21</smiles>

6e

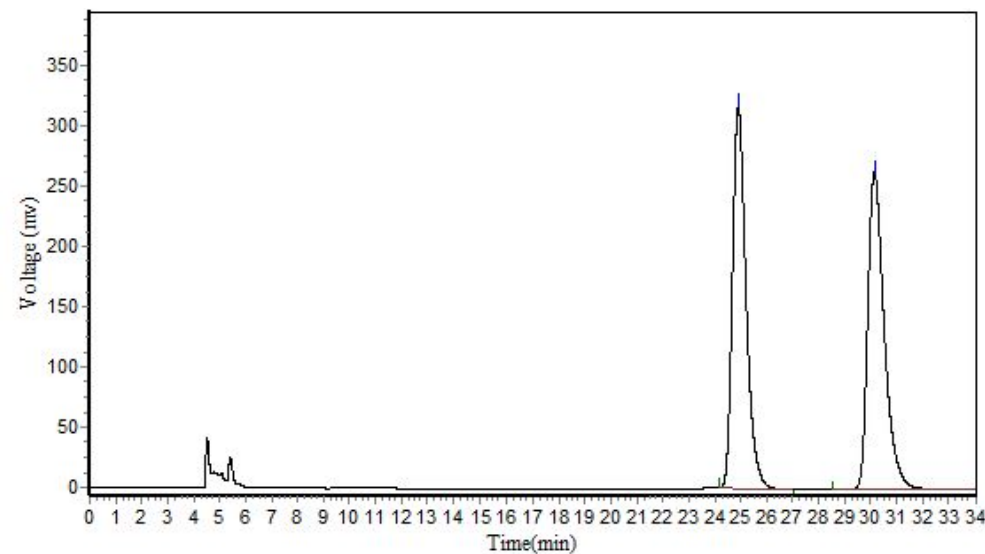

Results

\begin{tabular}{|c|c|c|c|c|c|}
\hline Peak No. & Peak ID & Ret Time & Height & Area & Conc. \\
\hline 1 & & 24.923 & 316231.969 & 11577257.000 & 49.8511 \\
\hline 2 & & 30.190 & 262550.938 & 11646399.000 & 50.1489 \\
\hline Total & & & 578782.906 & 23223656.000 & 100.000 \\
\hline
\end{tabular}

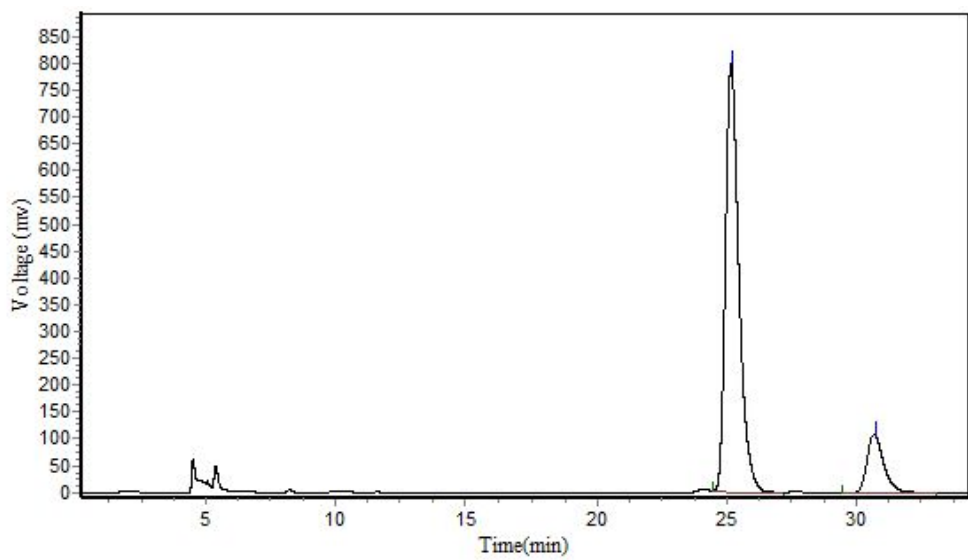

Results

\begin{tabular}{|c|c|c|c|c|c|}
\hline Peak No. & Peak ID & Ret Time & Height & Area & Conc. \\
\hline 1 & & 25.198 & 796518.938 & 28825336.000 & 85.5814 \\
\hline 2 & & 30.732 & 109109.625 & 4856428.500 & 14.4186 \\
\hline Total & & & 905628.563 & 33681764.500 & 100.0000 \\
\hline
\end{tabular}


(S)-methyl -6-fluoro-1-hydroxy-3-phenyl-1H-indene-2-carboxylate (5f)<smiles>COC(=O)C1=C(P)c2ccc(F)cc2[C@H]1O</smiles>

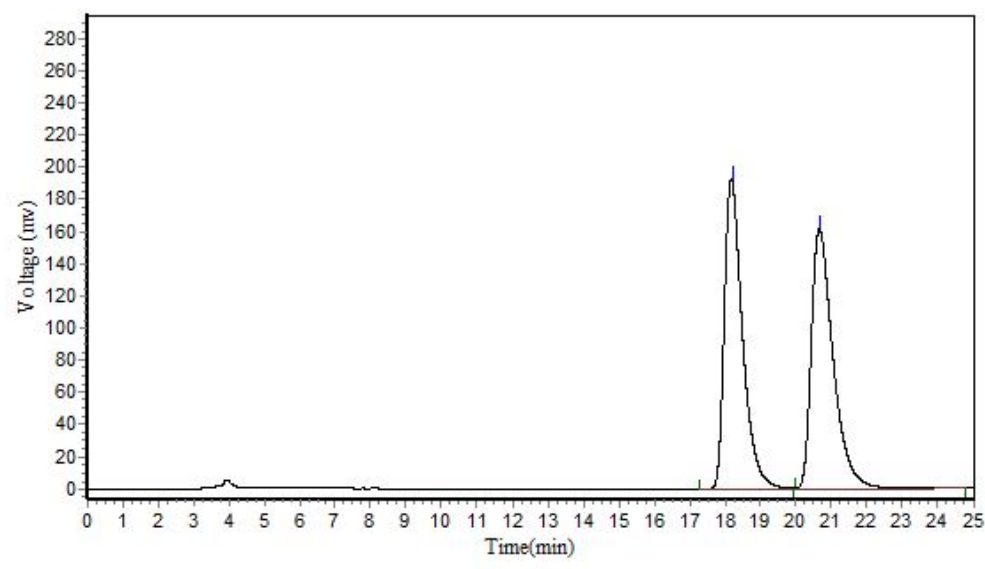

Results

\begin{tabular}{cccccc} 
Peak No. & Peak ID & Ret Time & Height & Area & Conc. \\
\hline 1 & 18.198 & 192707.828 & 6883036.500 & 49.7746 \\
2 & 20.698 & 161502.922 & 6945364.500 & 50.2254 \\
\hline Total & & 354210.750 & 13828401.000 & 100.0000
\end{tabular}

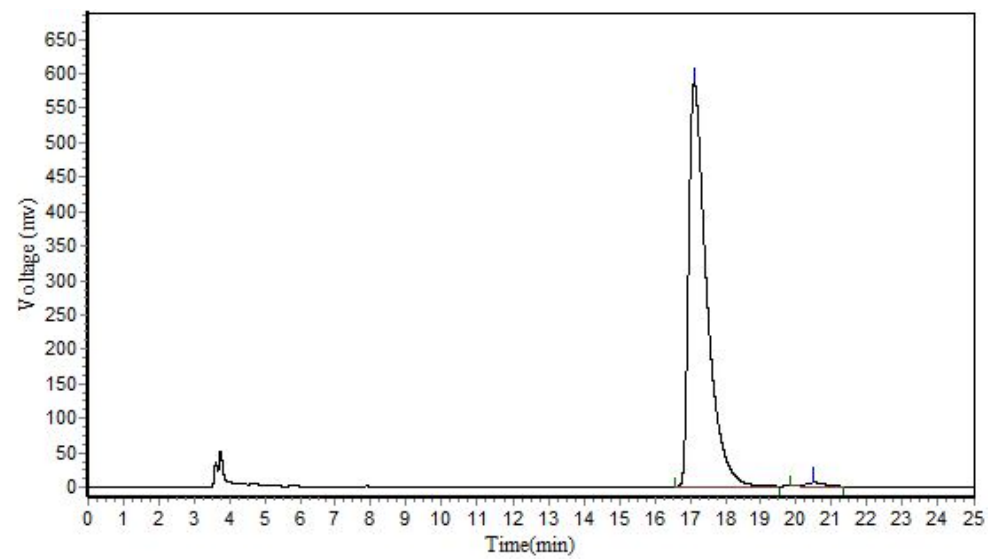

Results

\begin{tabular}{|c|c|c|c|c|c|}
\hline Peak No. & Peak ID & Ret Time & Height & Area & Conc. \\
\hline 1 & & 17.115 & 587010.625 & 20725054.000 & 99.0052 \\
\hline 2 & & 20.483 & 5453.474 & 208238.391 & 0.9948 \\
\hline Total & & & 592464.099 & 20933292.391 & 100.000 \\
\hline
\end{tabular}


(S)-methyl-6-fluoro-1-hydroxy-2-phenyl-1H-indene-3-carboxylate (6f)<smiles>CC(=O)C1=C(c2ccccc2)[C@H](O)c2cc(F)ccc21</smiles>

6f

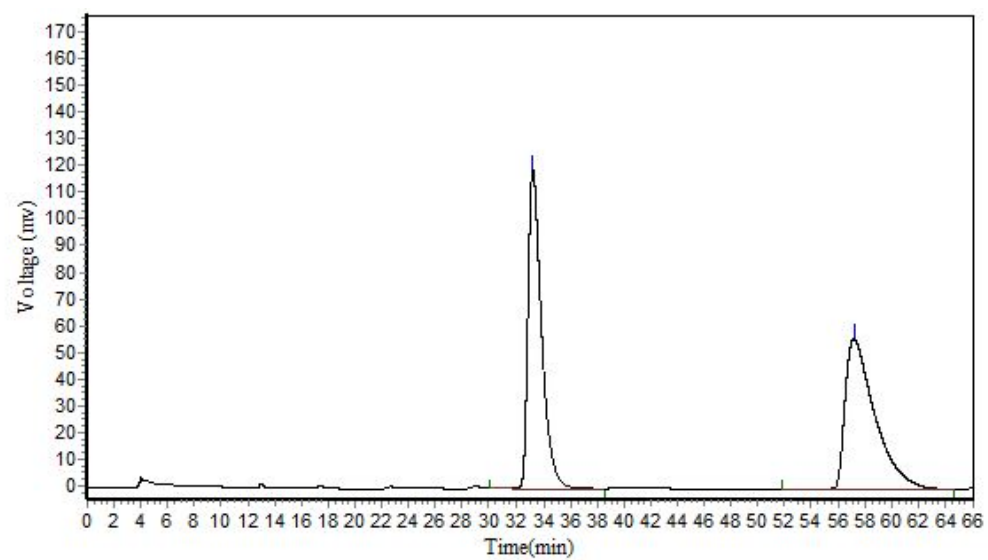

Results

\begin{tabular}{|c|c|c|c|c|c|}
\hline Peak No. & Peak ID & Ret Time & Height & Area & Conc. \\
\hline 1 & & 33.198 & 119254.766 & 8600531.000 & 49.7294 \\
\hline 2 & & 57.198 & 55998.688 & 8694122.000 & 50.2706 \\
\hline Total & & & 175253.453 & 17294653.000 & 100.0000 \\
\hline
\end{tabular}

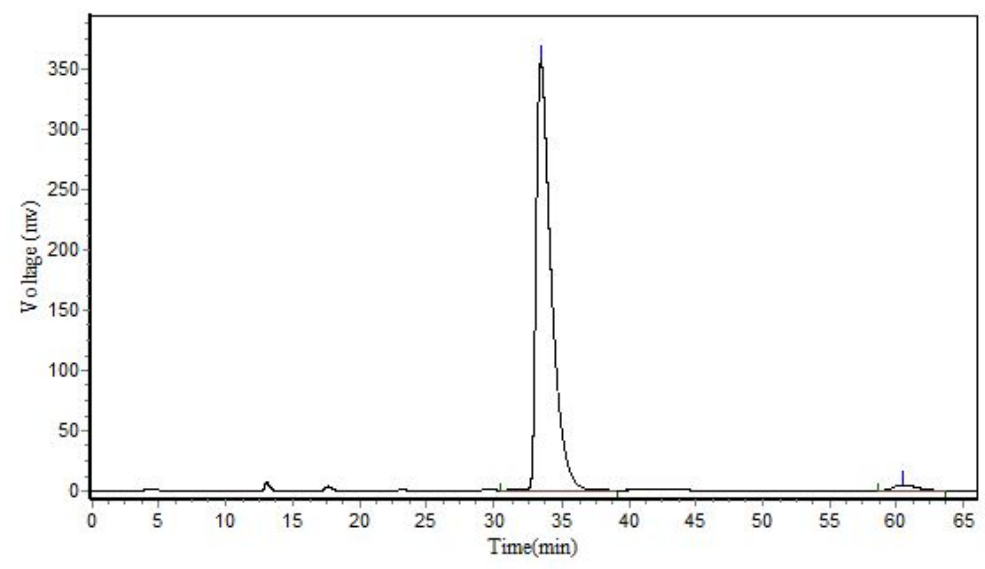

Results

\begin{tabular}{|c|c|c|c|c|c|}
\hline \multicolumn{6}{|c|}{. } \\
\hline Peak No. & Peak ID & Ret Time & Height & Area & Conc. \\
\hline 1 & & 33.422 & 356236.375 & 27867812.000 & 97.8694 \\
\hline 2 & & 60.423 & 4813.526 & 606678.000 & 2.1306 \\
\hline Total & & & 361049.901 & 28474490.000 & $100.000 \mathrm{C}$ \\
\hline
\end{tabular}


(R)-2-ethyl-3-phenyl-1H-inden-1-ol (5g); (S)-3-ethyl-2-phenyl-1H-inden-1-ol (6g)<smiles>CCC1=C(c2ccccc2)c2ccccc2[C@H]1O</smiles>

$5 \mathrm{~g}$<smiles>CCC1=C(c2ccccc2)[C@H](O)c2ccccc21</smiles>

$6 \mathrm{~g}$

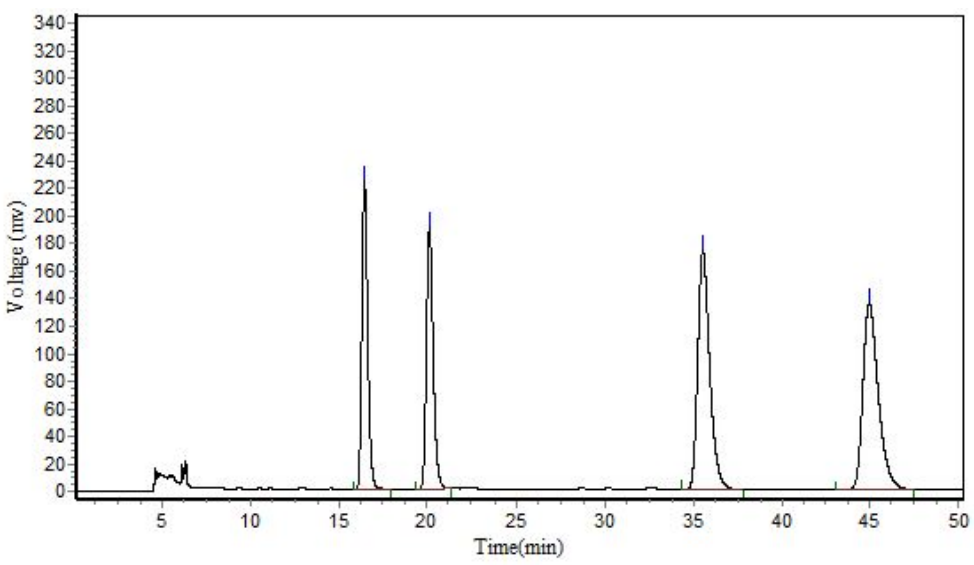

Results

\begin{tabular}{|c|c|c|c|c|c|}
\hline Peak No. & Peak ID & Ret Time & Height & Area & Conc. \\
\hline 1 & & 16.457 & 224045.828 & 5123475.000 & 19.3665 \\
\hline 2 & & 20.132 & 189789.438 & 5065745.500 & 19.1483 \\
\hline 3 & & 35.523 & 173188.438 & 8105785.000 & 30.6396 \\
\hline 4 & & 44.948 & 134973.938 & 8160282.500 & 30.8456 \\
\hline Total & & & 721997.641 & 26455288.000 & 100.0000 \\
\hline
\end{tabular}

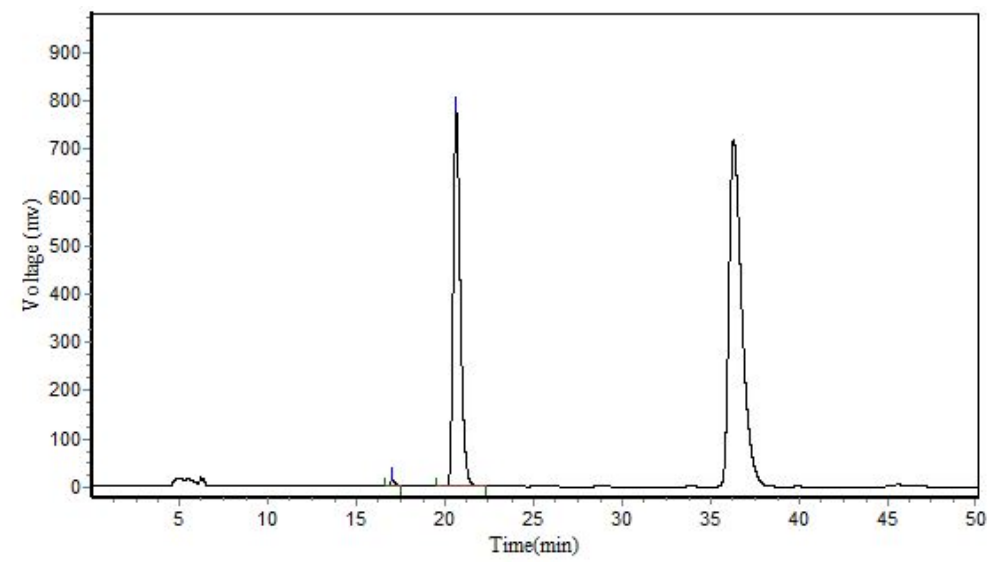

Results

\begin{tabular}{|c|c|c|c|c|c|}
\hline Peak No. & Peak ID & Ret Time & Height & Area & Conc. \\
\hline 1 & & 17.037 & 13060.229 & 280144.188 & 1.2619 \\
\hline 2 & & 20.682 & 778545.125 & 21920666.000 & 98.7381 \\
\hline Total & & & 791605.354 & 22200810.188 & 100.0000 \\
\hline
\end{tabular}




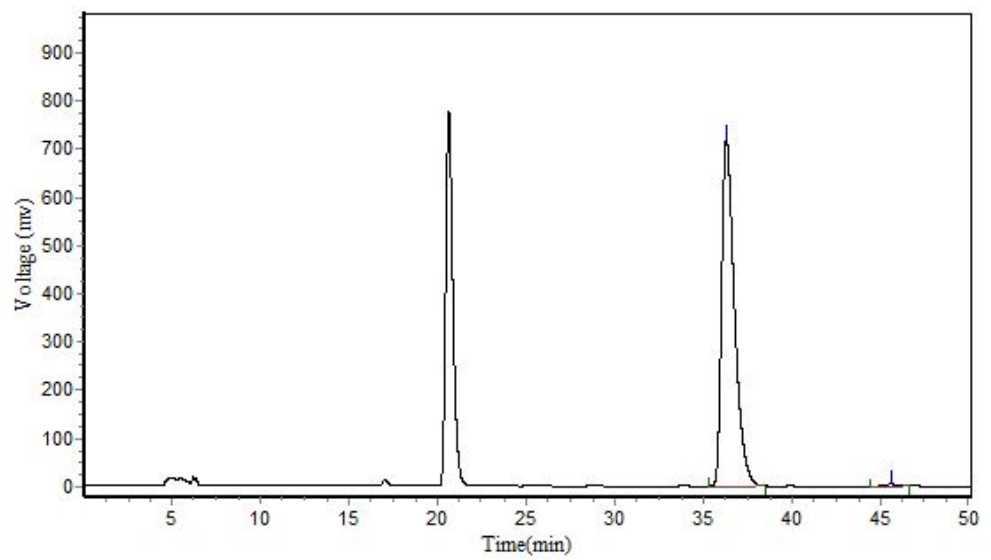

\begin{tabular}{|c|c|c|c|c|c|}
\hline \multicolumn{6}{|c|}{ Results } \\
\hline Peak No. & Peak ID & Ret Time & Height & Area & Conc. \\
\hline 1 & & 36.302 & 720290.625 & 36435264.000 & 99.4447 \\
\hline 2 & & 45.612 & 3865.943 & 203443.688 & 0.5553 \\
\hline Total & & & 724156.568 & 36638707.688 & 100.0000 \\
\hline
\end{tabular}


(S)-2,3-bis(methoxymethyl)-1H-inden-1-ol (5h)<smiles>O=[N+]([O-])C1=C([N+]([O-])O[Na])c2ccccc2[C@H]1O</smiles>

$5 \mathrm{~h}$

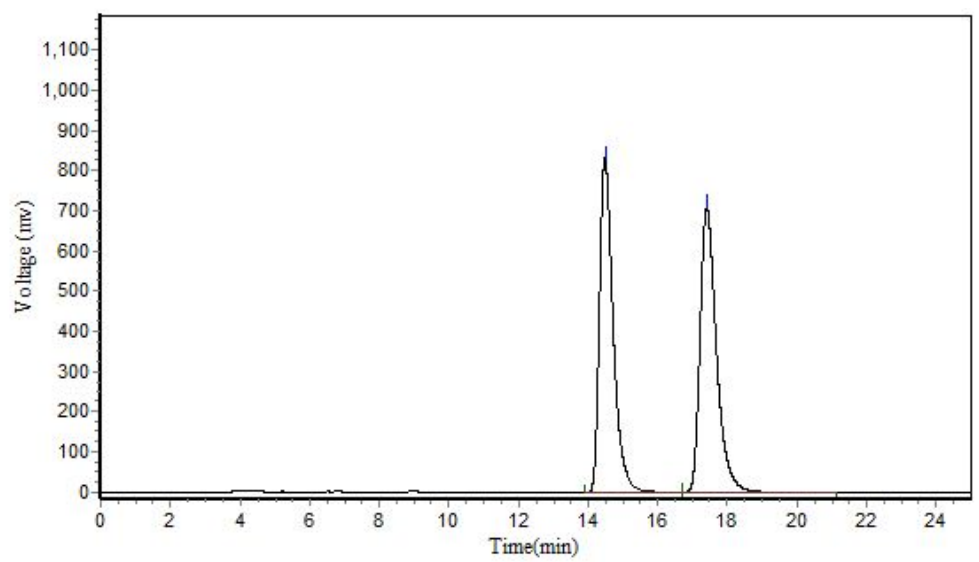

Results

\begin{tabular}{|c|c|c|c|c|c|}
\hline Peak No. & Peak ID & Ret Time & Height & Area & Conc. \\
\hline 1 & & 14.498 & 833489.375 & 23064088.000 & 49.5263 \\
\hline 2 & & 17.432 & 709831.625 & 23505270.000 & 50.4737 \\
\hline Total & & & 1543321.000 & 46569358.000 & 100.000 \\
\hline
\end{tabular}

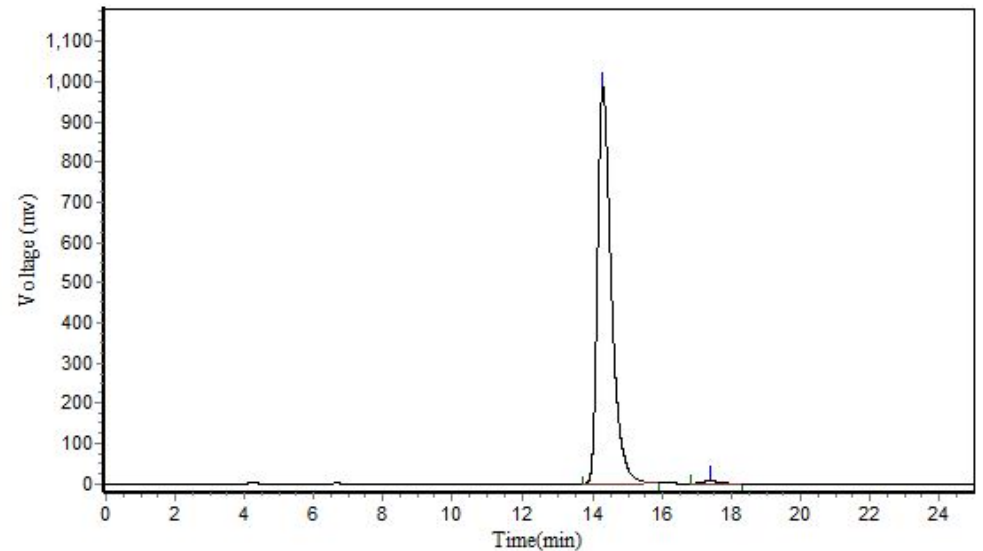

Results

\begin{tabular}{|c|c|c|c|c|c|}
\hline Peak No. & Peak ID & Ret Time & Height & Area & Conc. \\
\hline 1 & & 14.302 & 986183.000 & 27399276.000 & 99.2292 \\
\hline 2 & & 17.403 & 6742.596 & 212828.500 & 0.7708 \\
\hline Total & & & 992925.596 & 27612104.500 & 100.0000 \\
\hline
\end{tabular}


2. Copies of ${ }^{1} \mathrm{H}$ NMR and ${ }^{13} \mathrm{C}$ NMR spectra of products
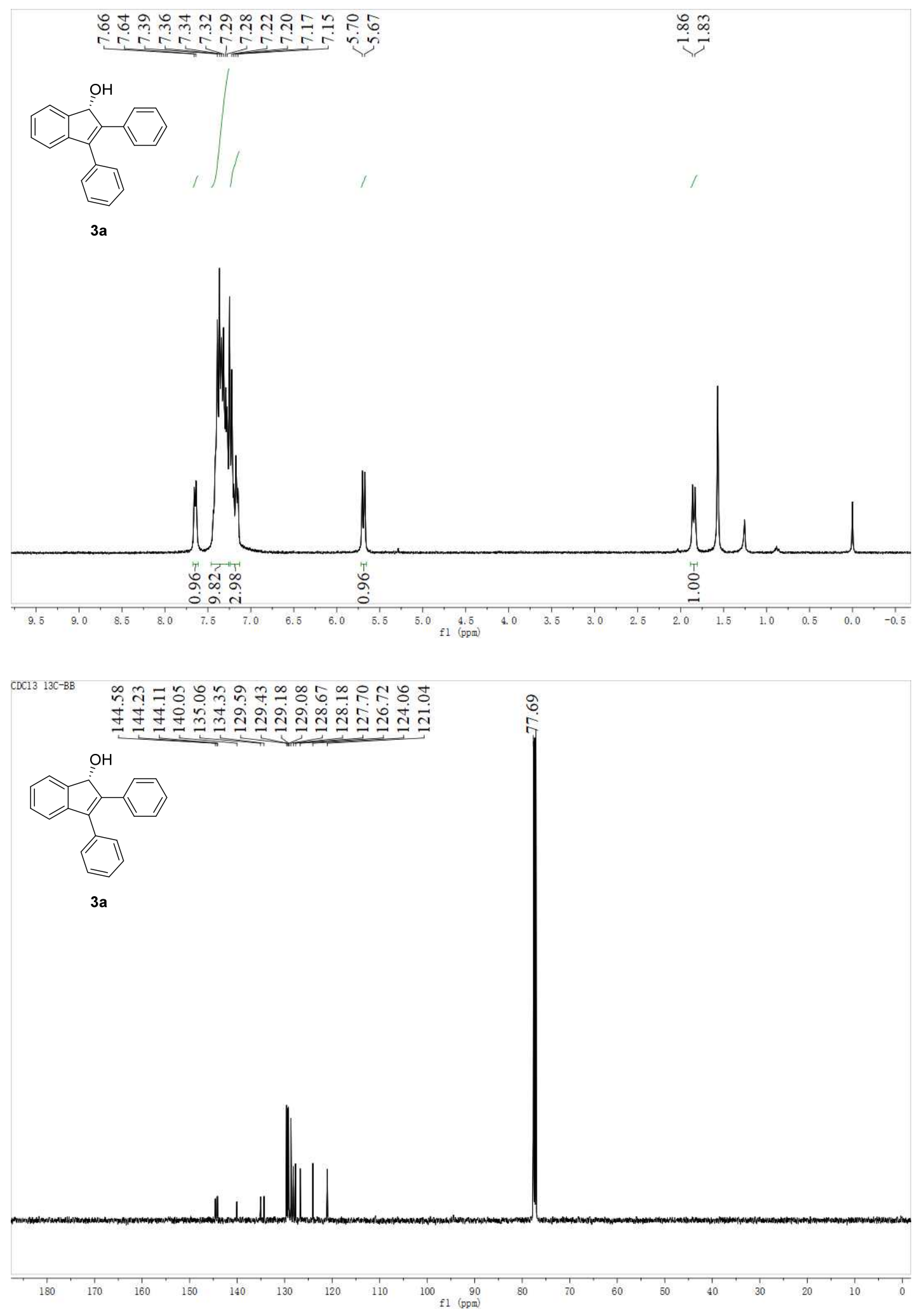

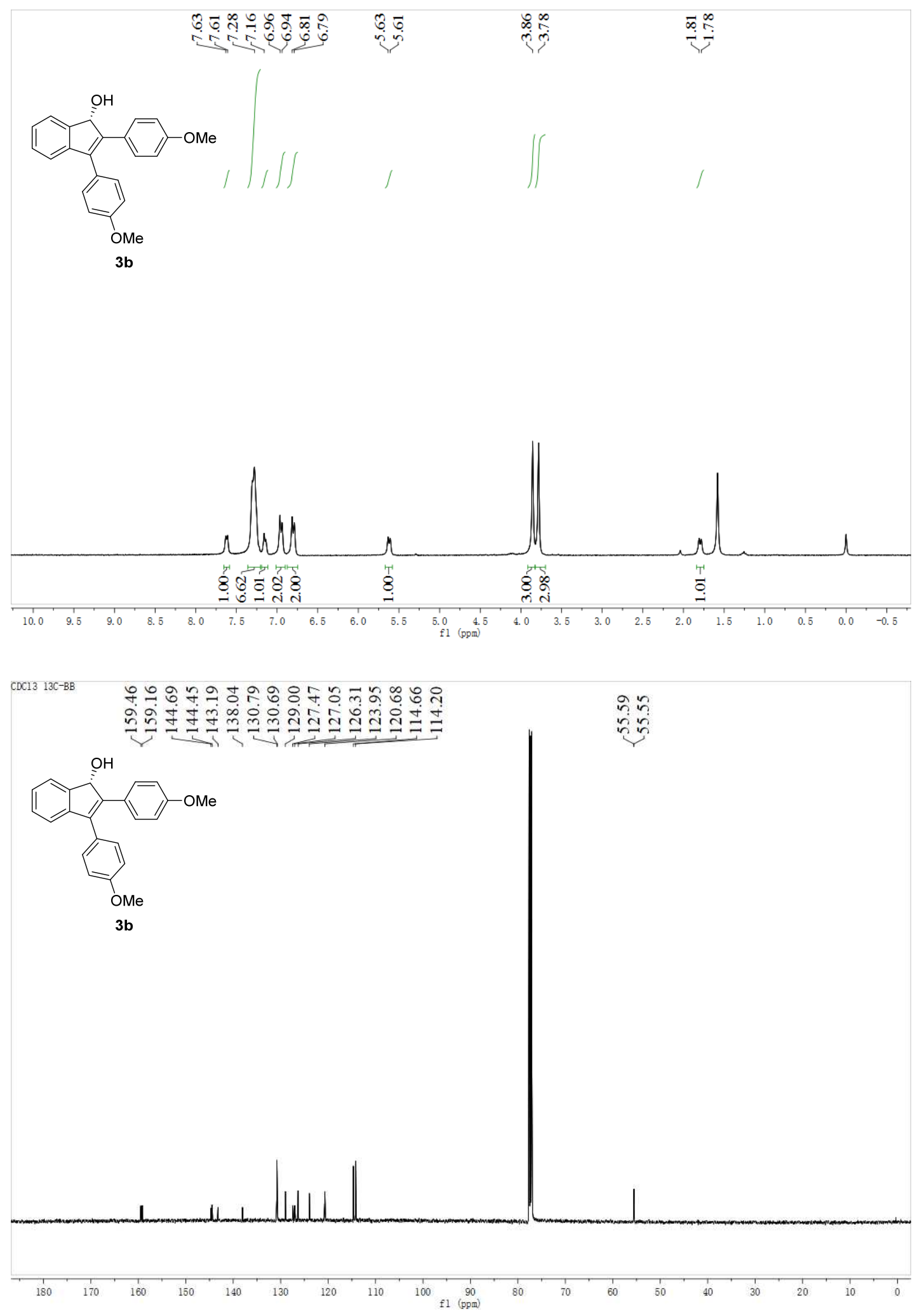


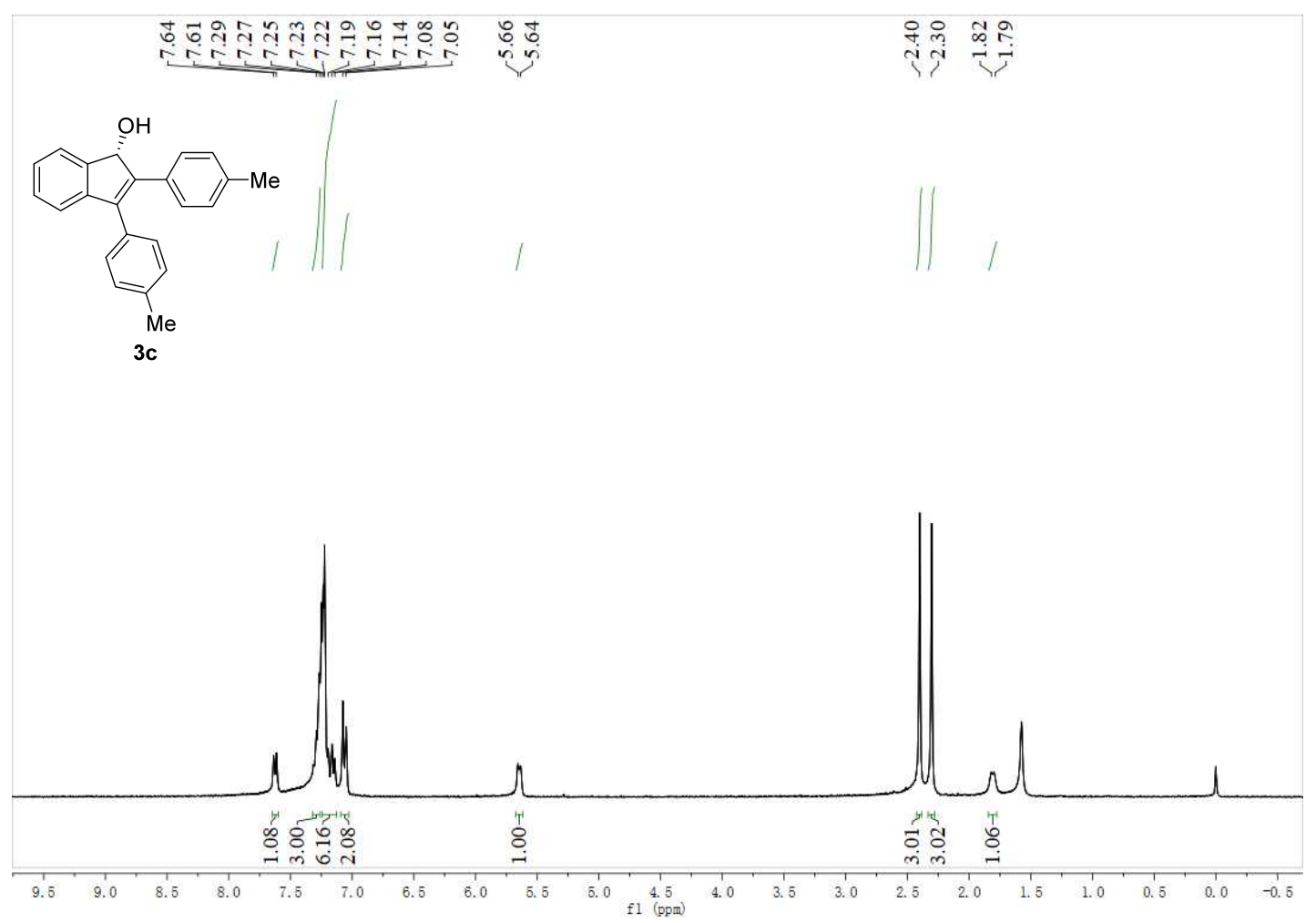

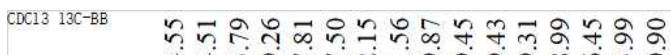

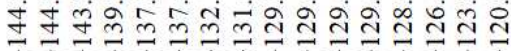

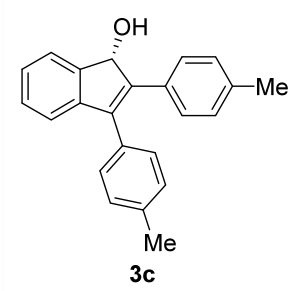

$\stackrel{5}{5}$

ำ

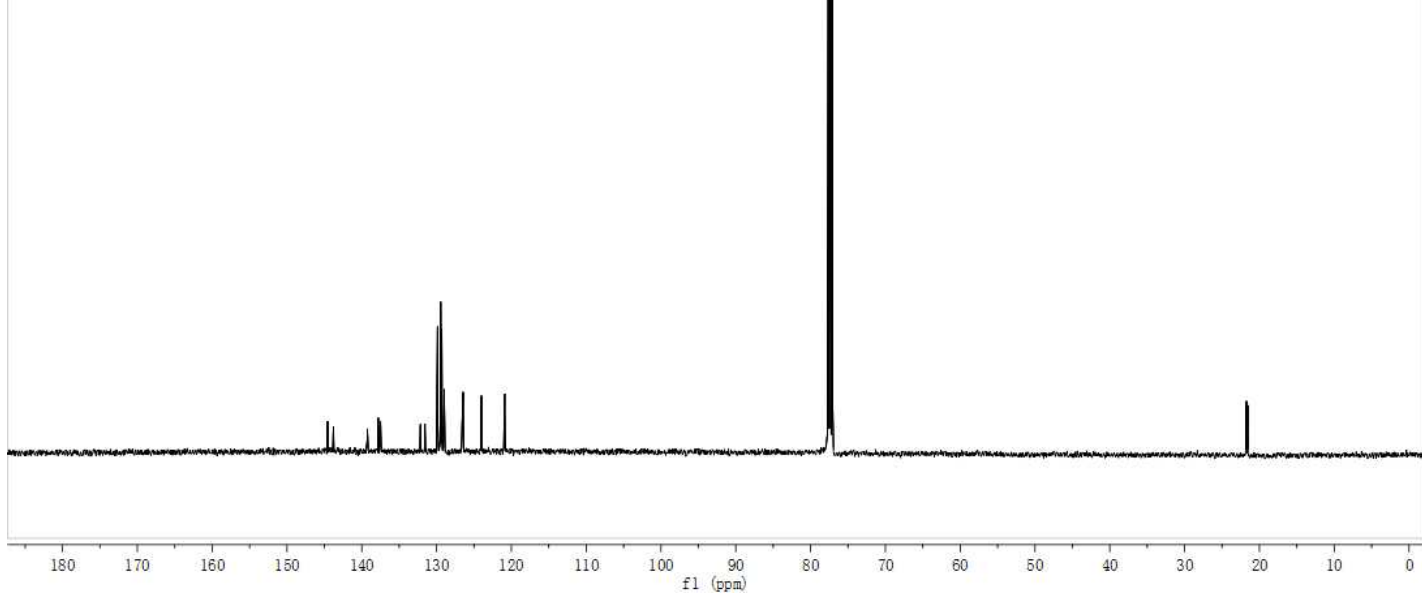



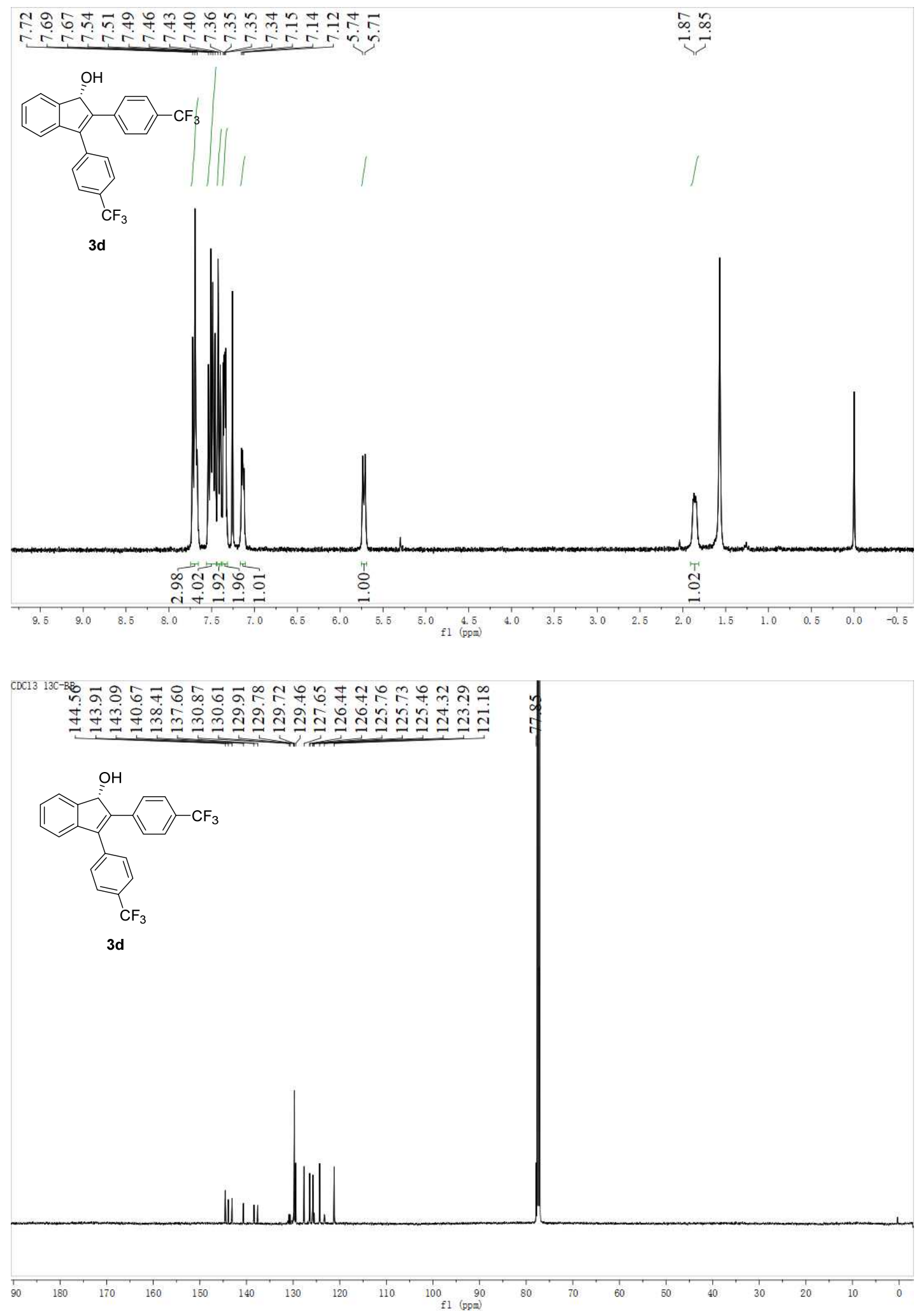

S34 

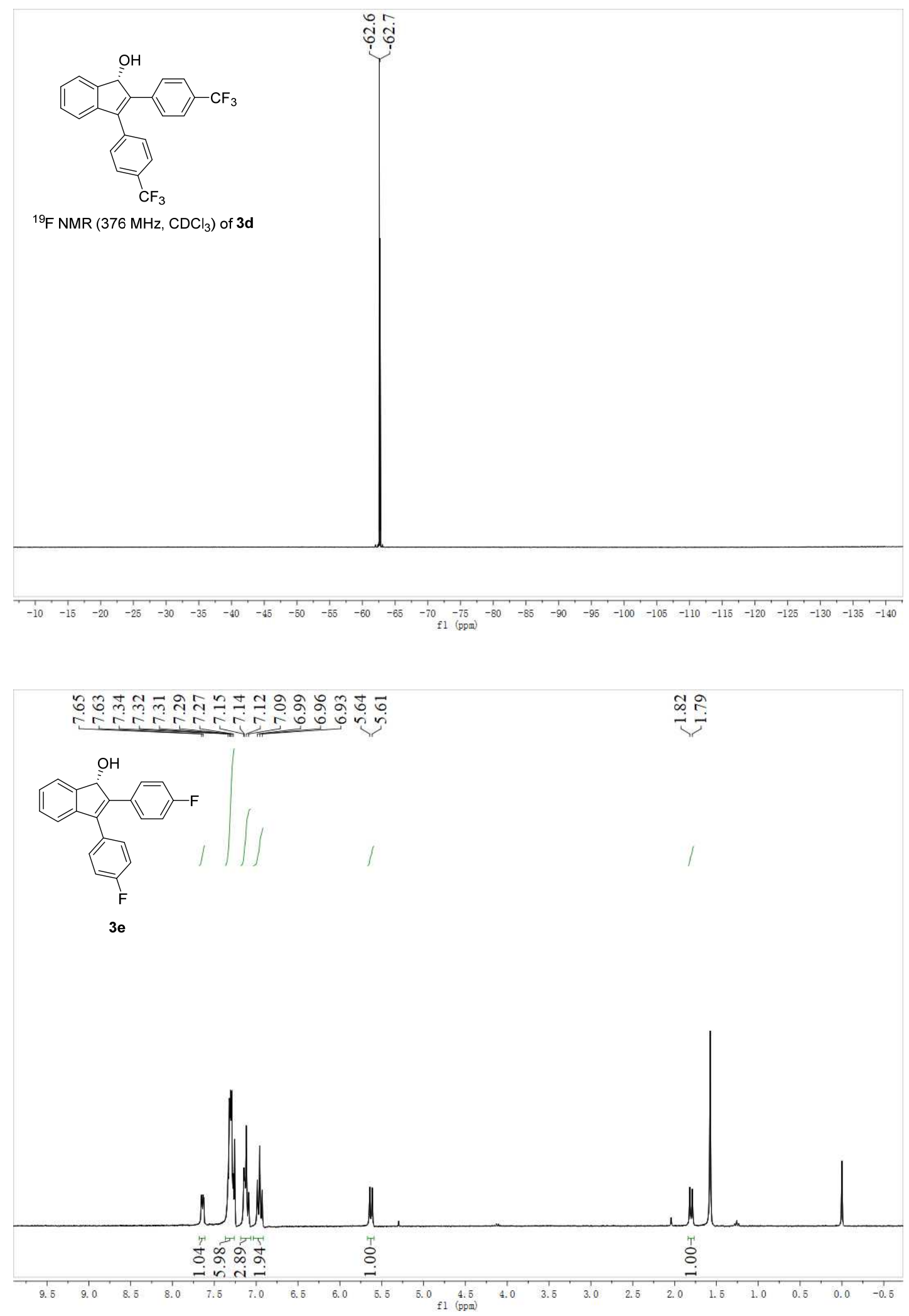

S35 

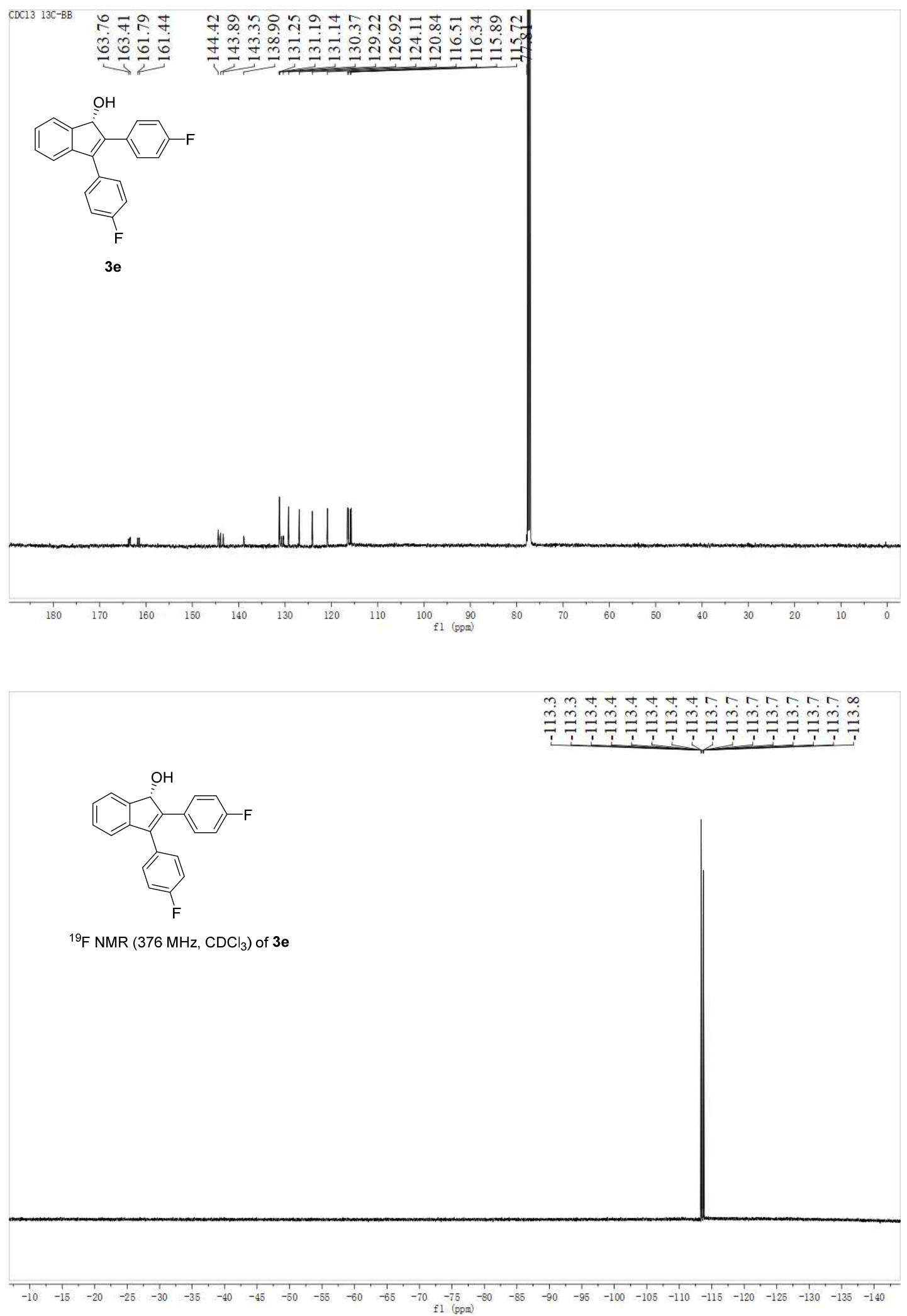


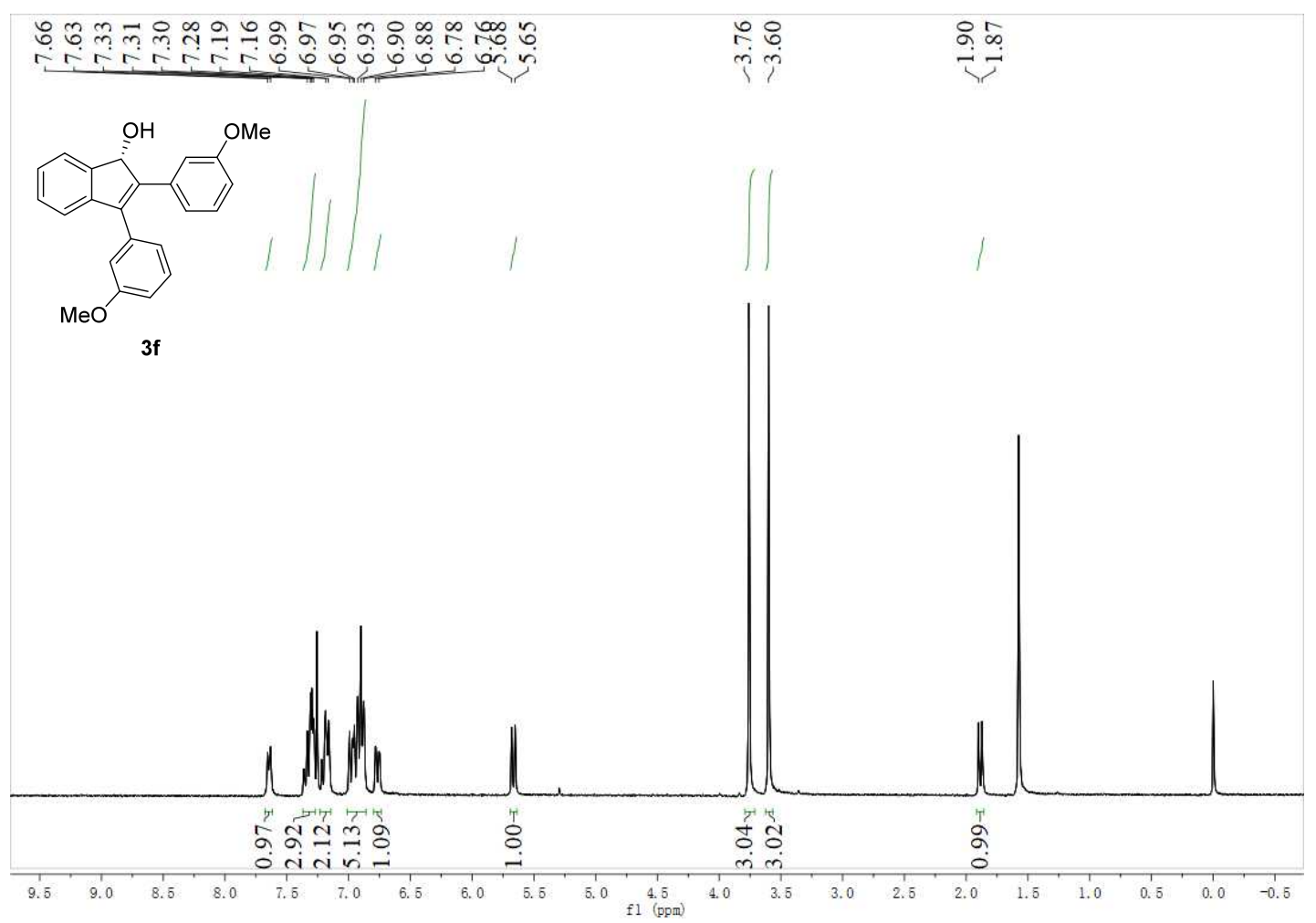

CDC13 $13 C-B B$

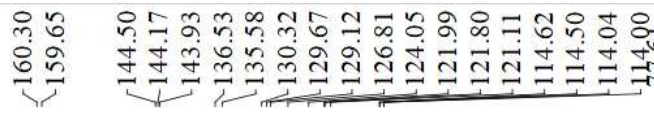
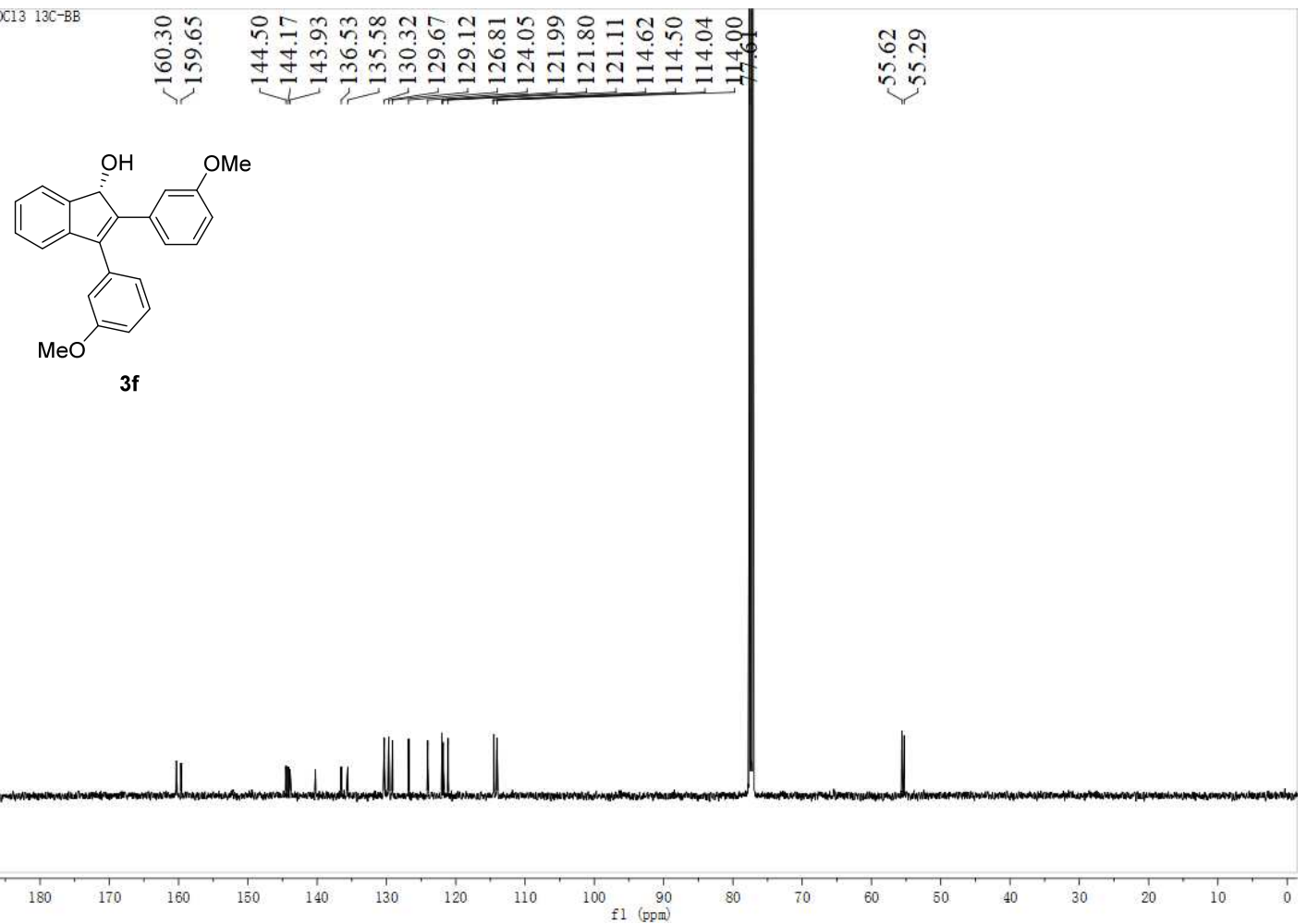


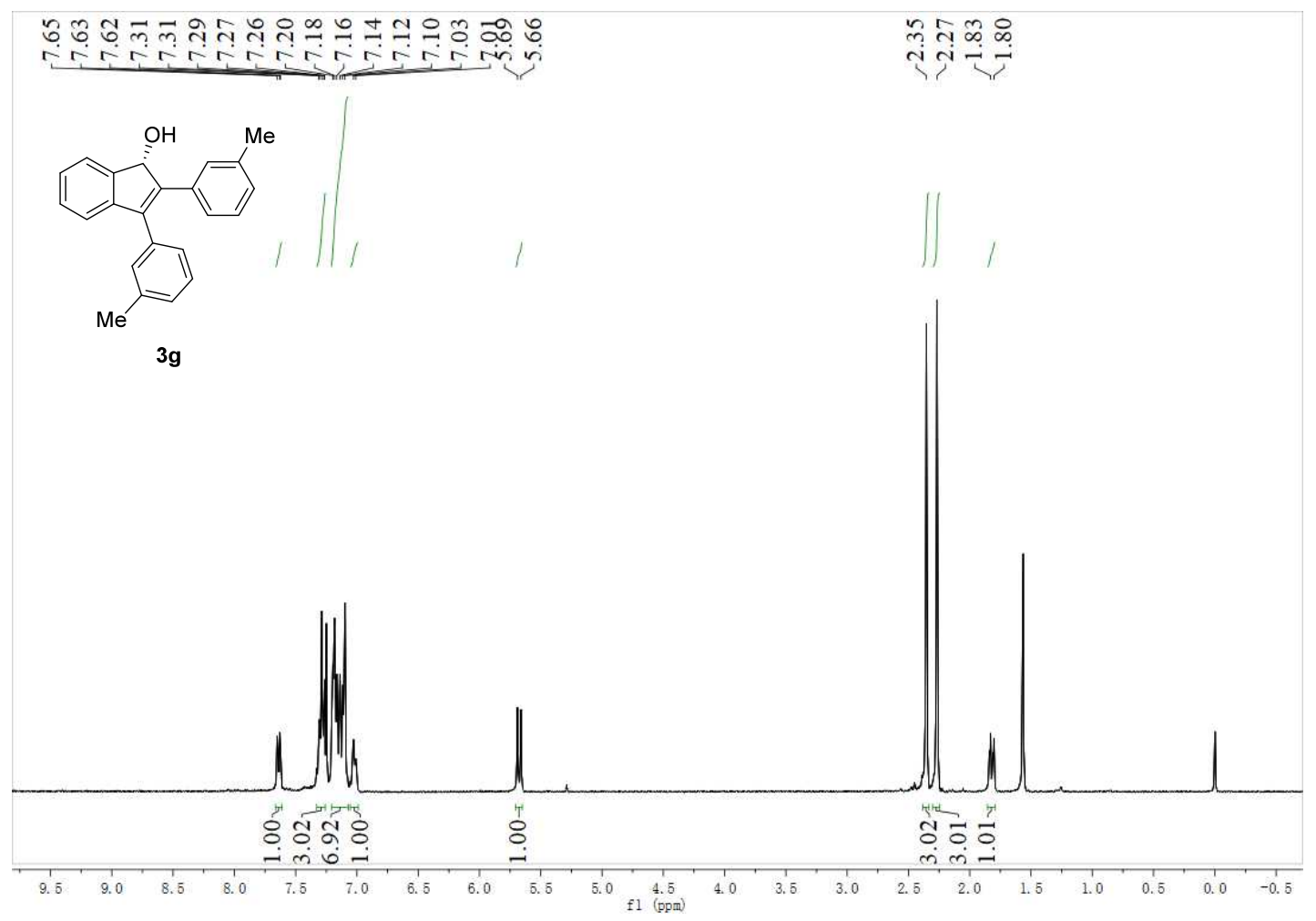

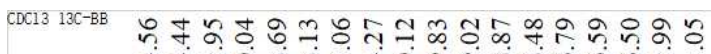

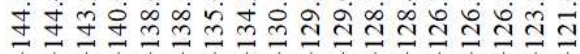
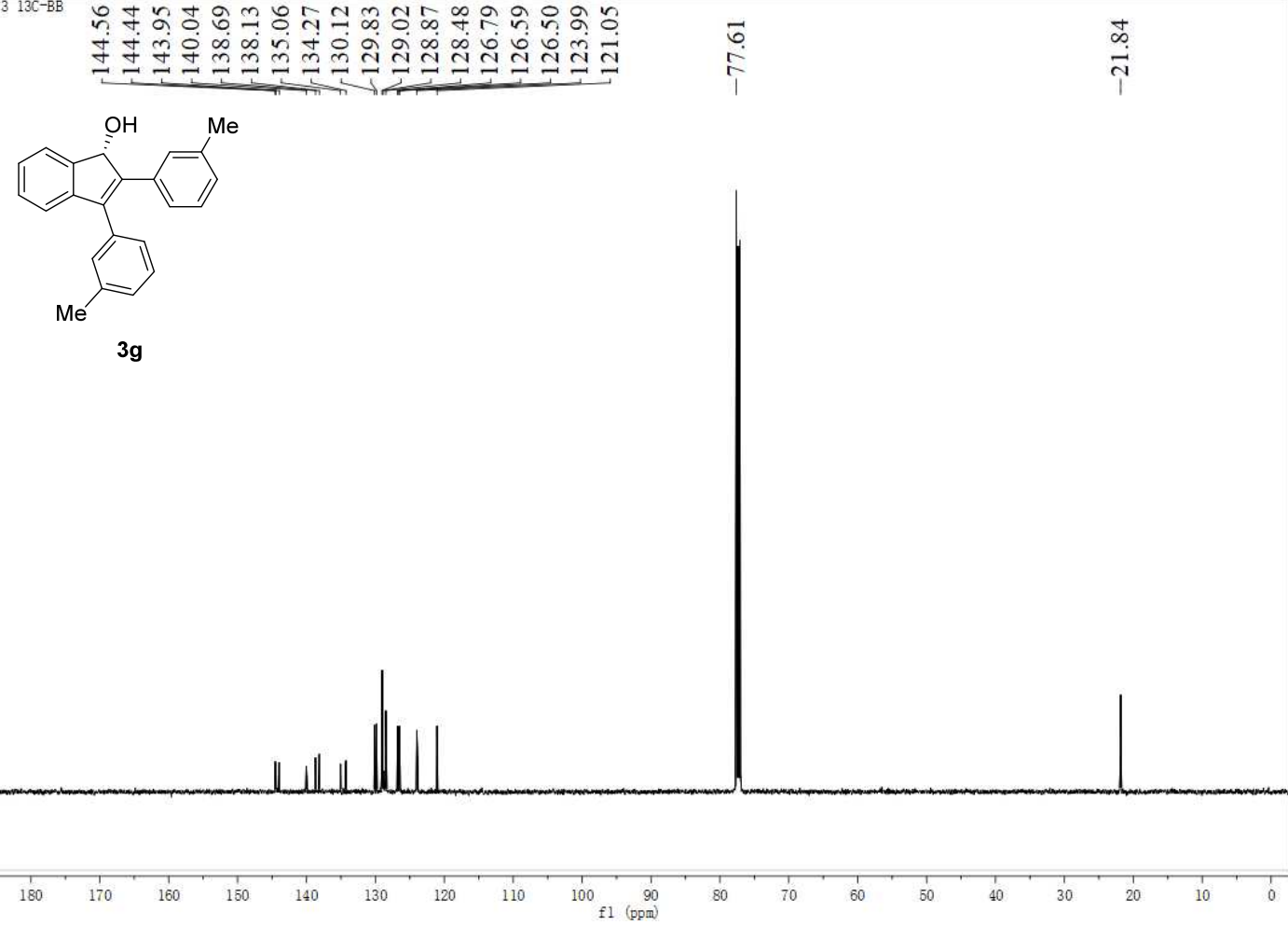


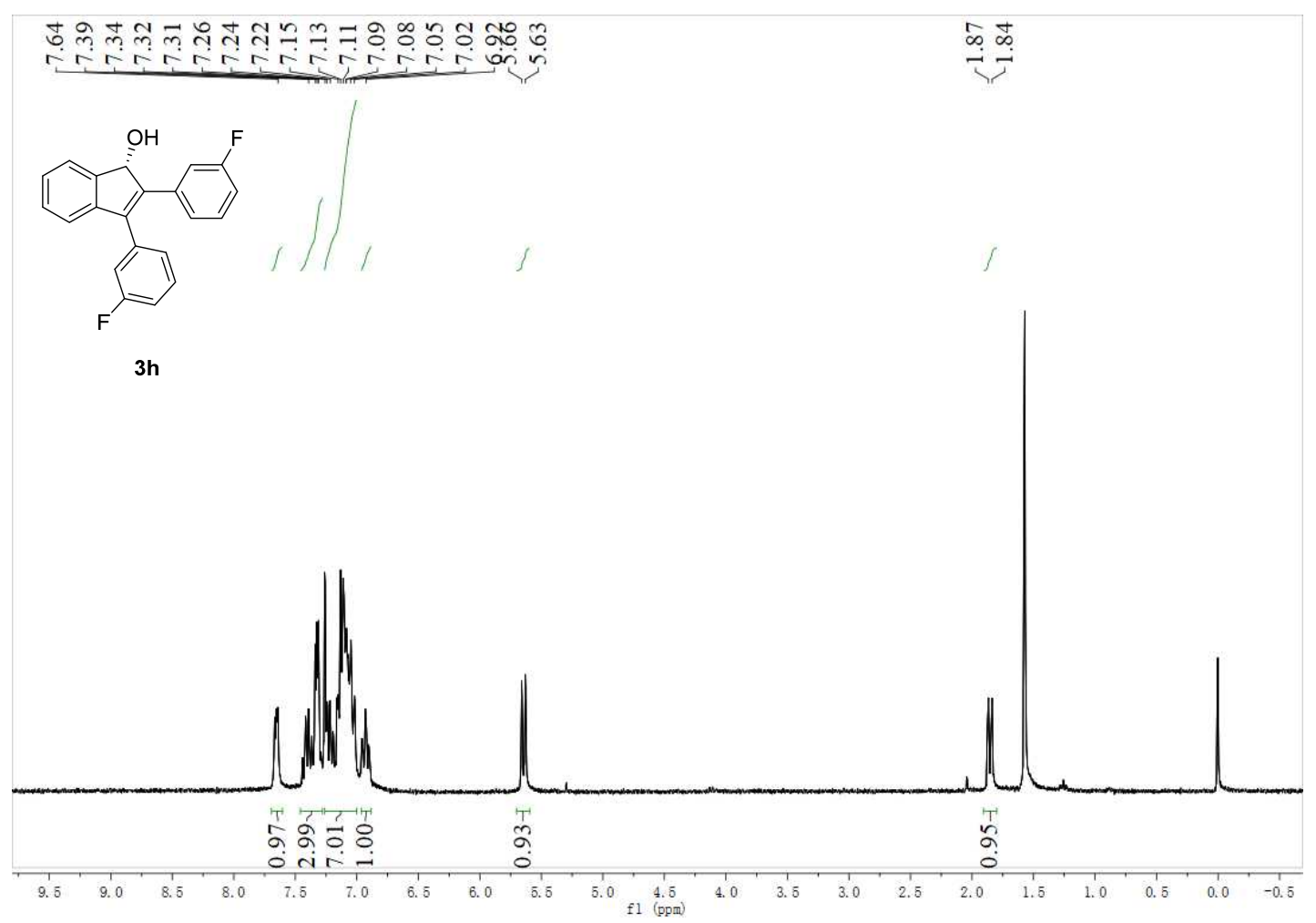

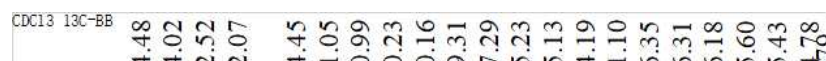

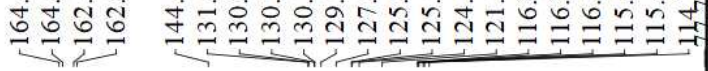

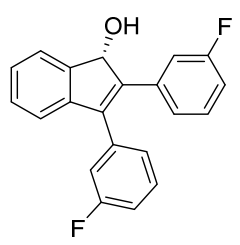

3h
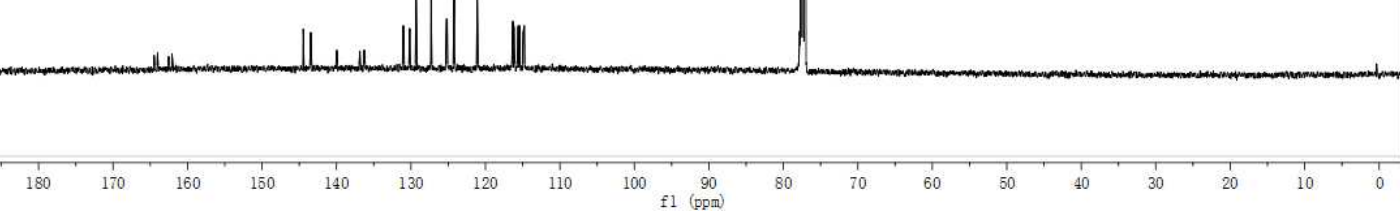


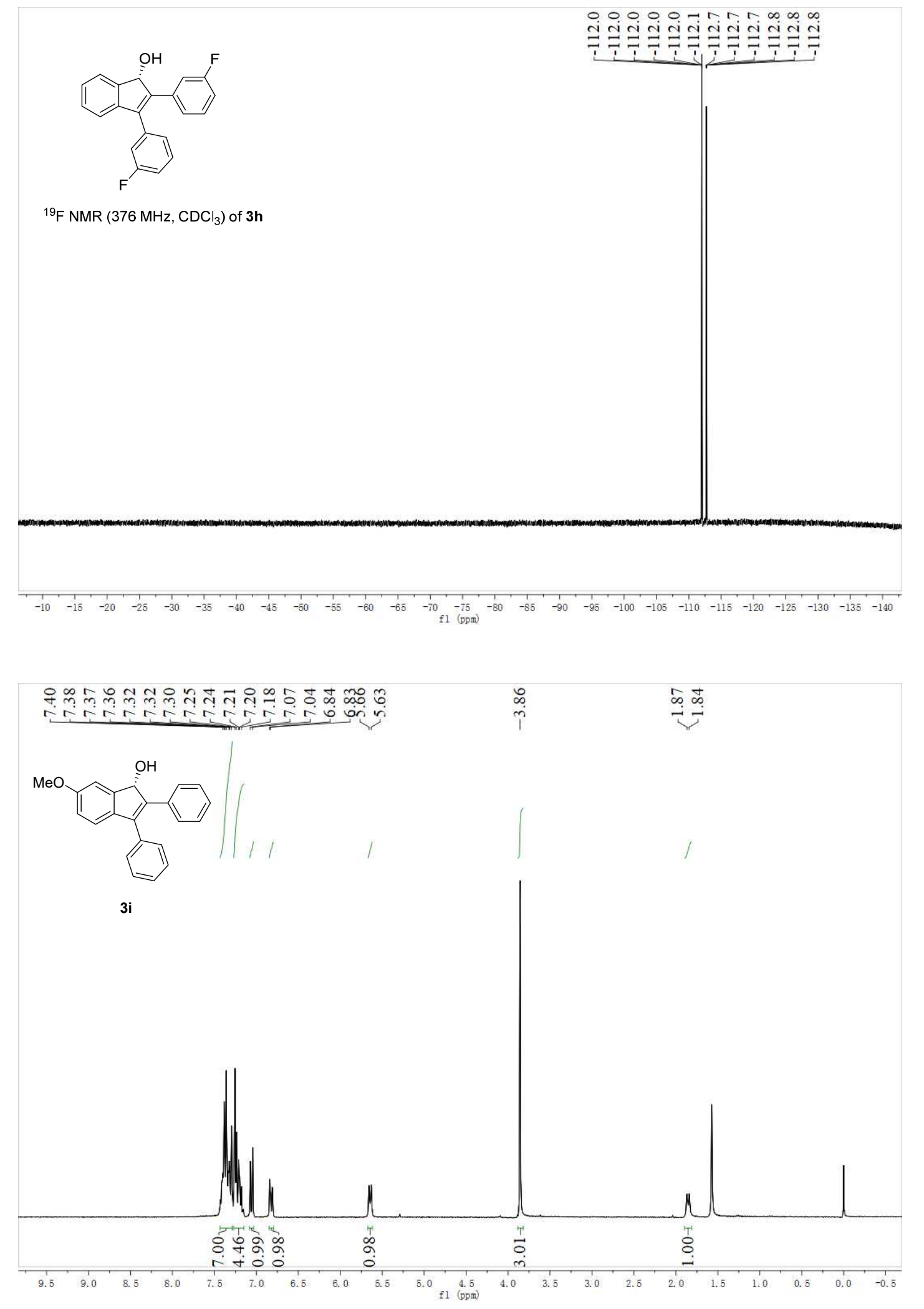



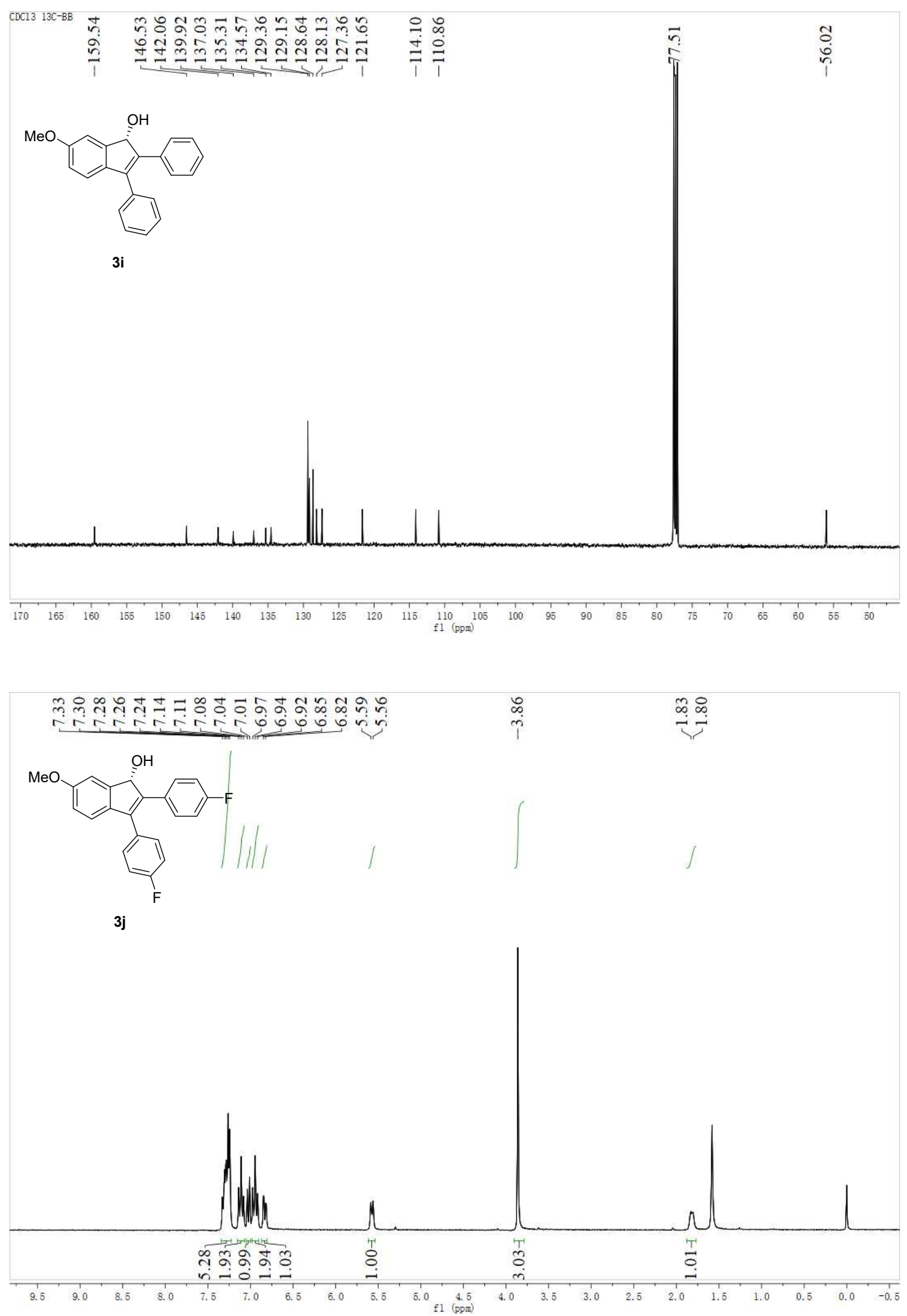

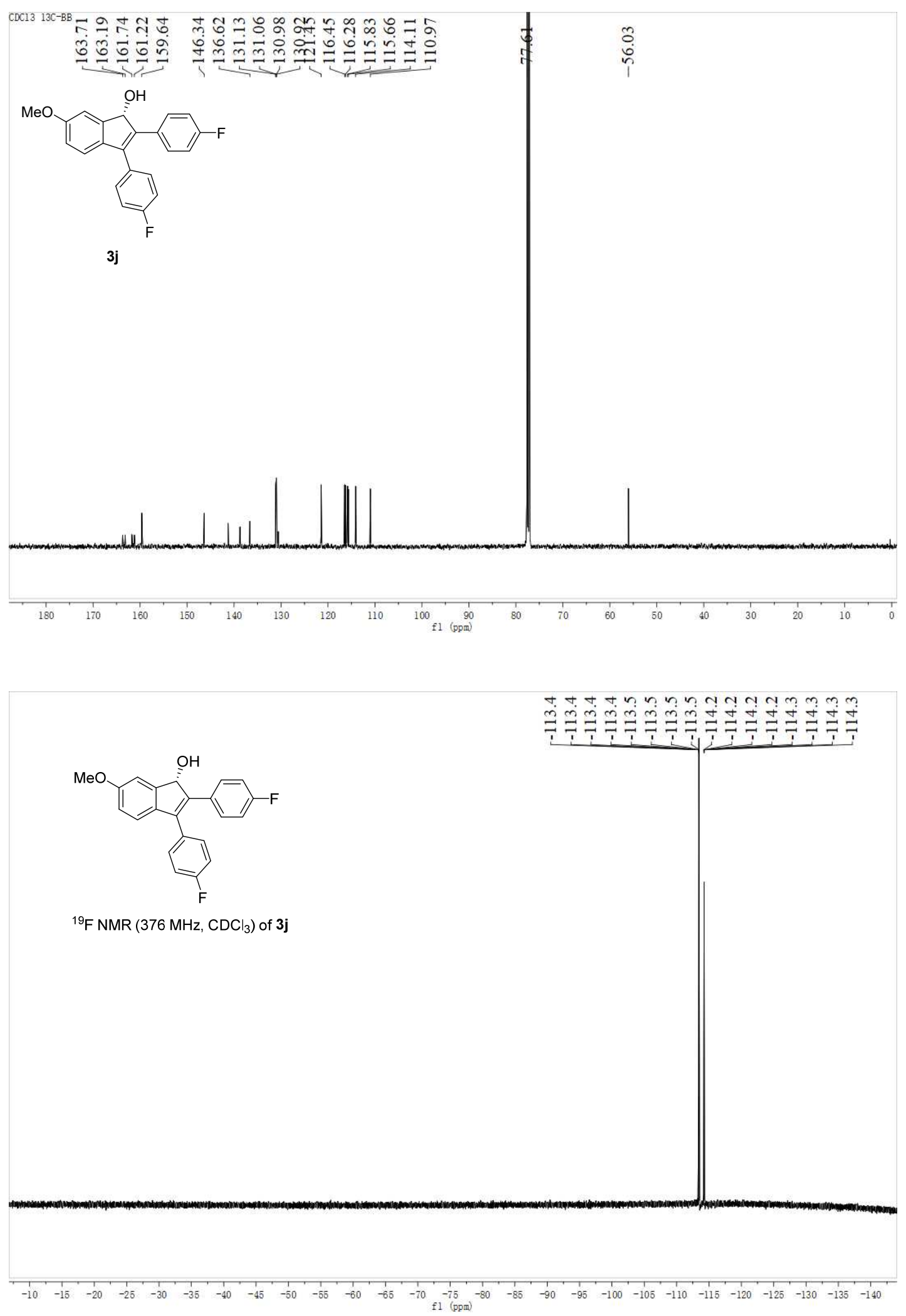

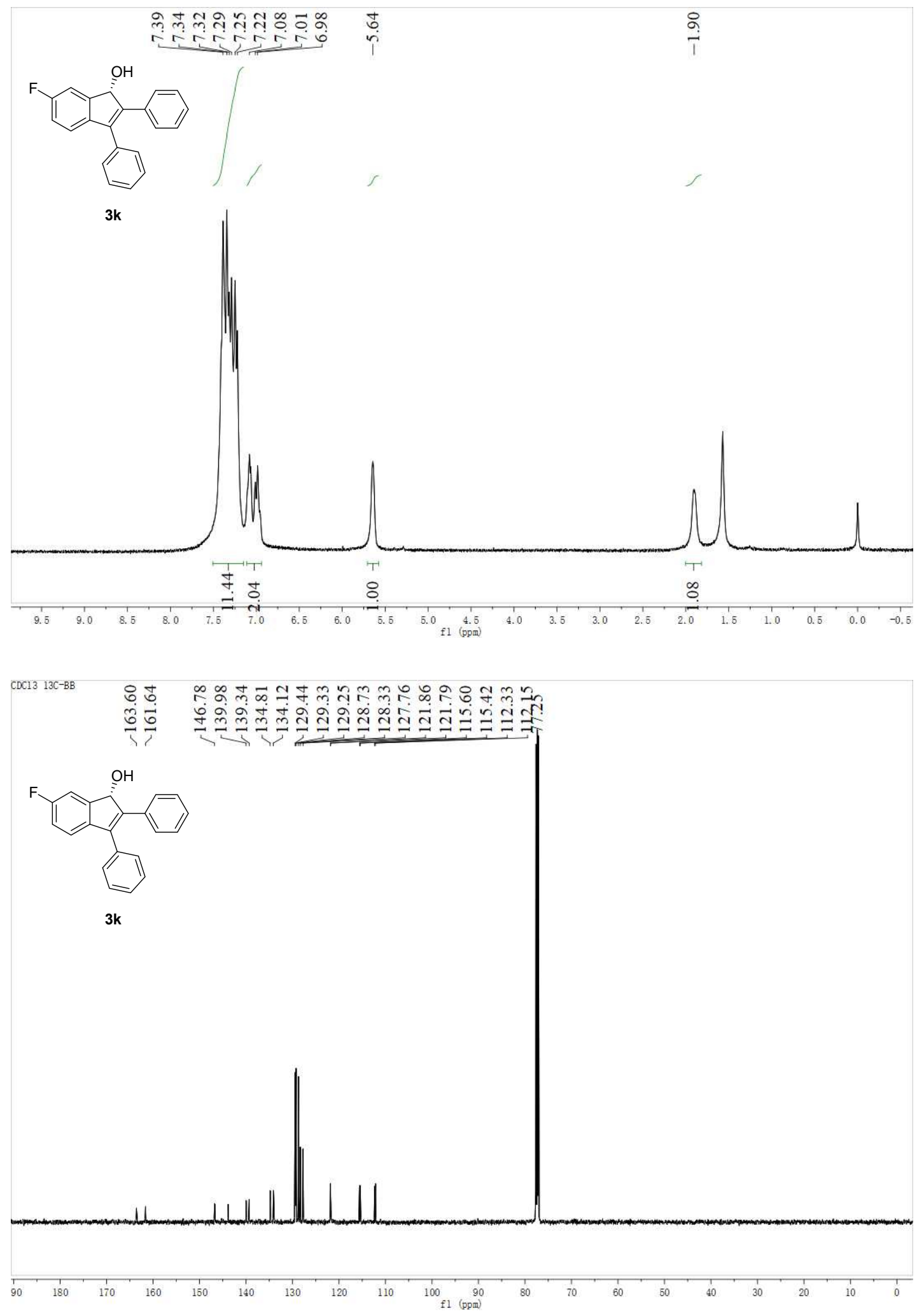


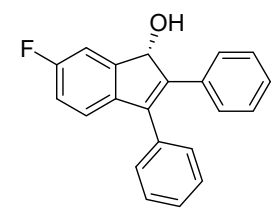

${ }^{19} \mathrm{~F} \mathrm{NMR}\left(376 \mathrm{MHz}, \mathrm{CDCl}_{3}\right)$ of $\mathbf{3 k}$

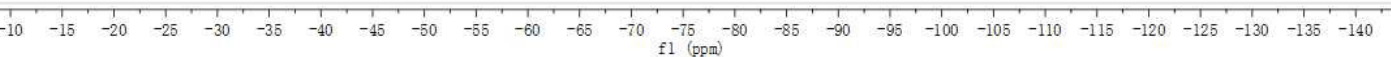

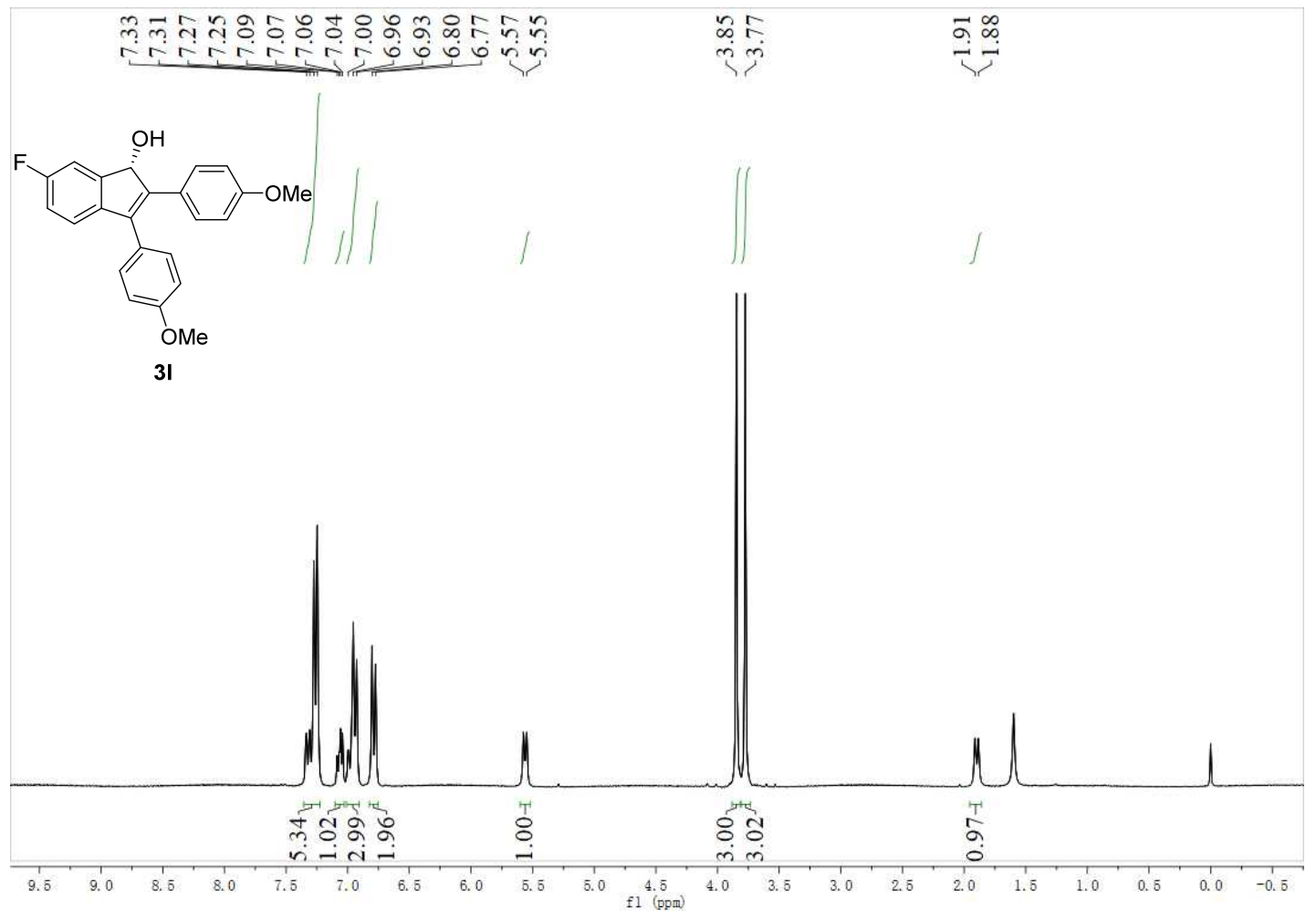



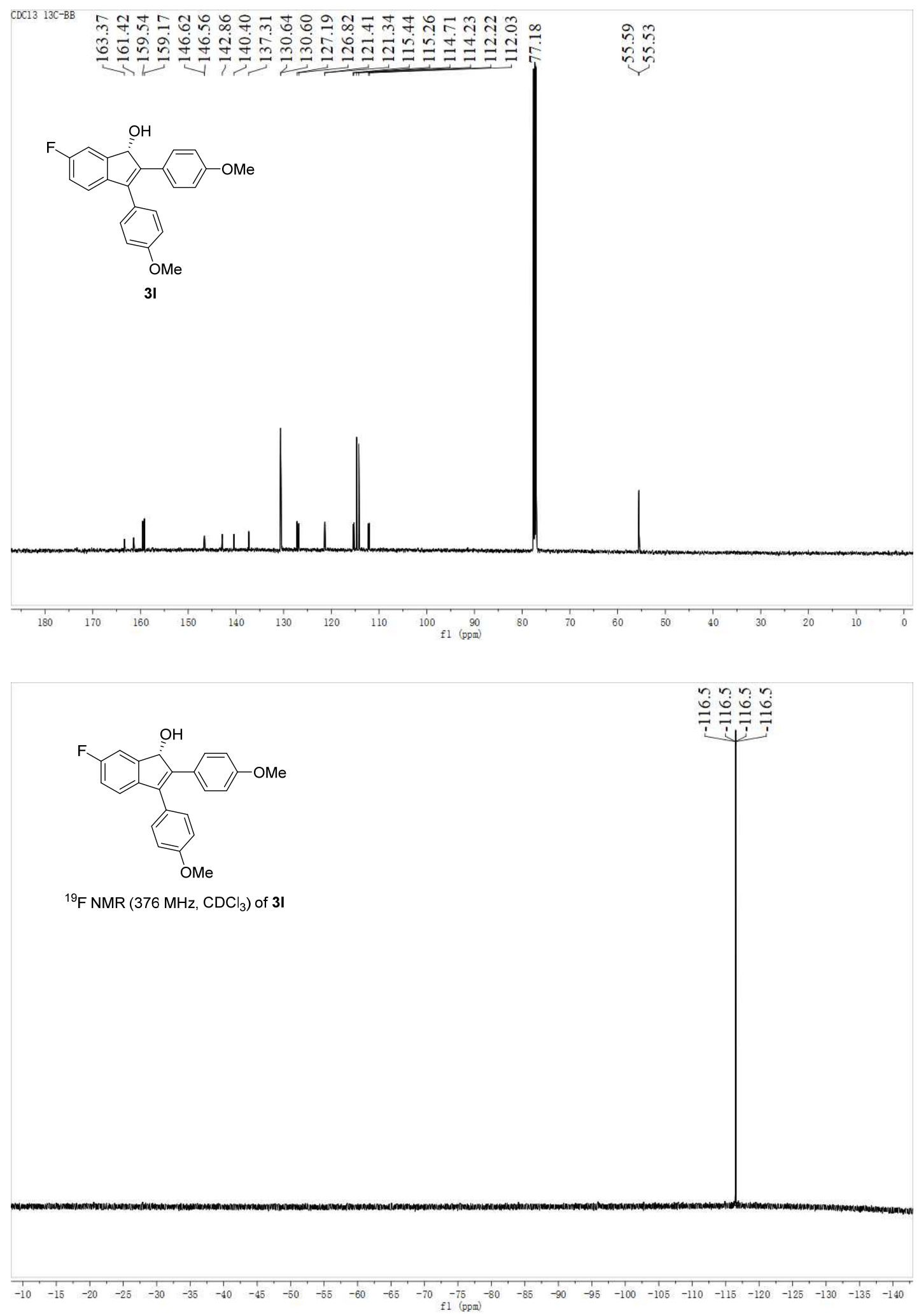

S45 

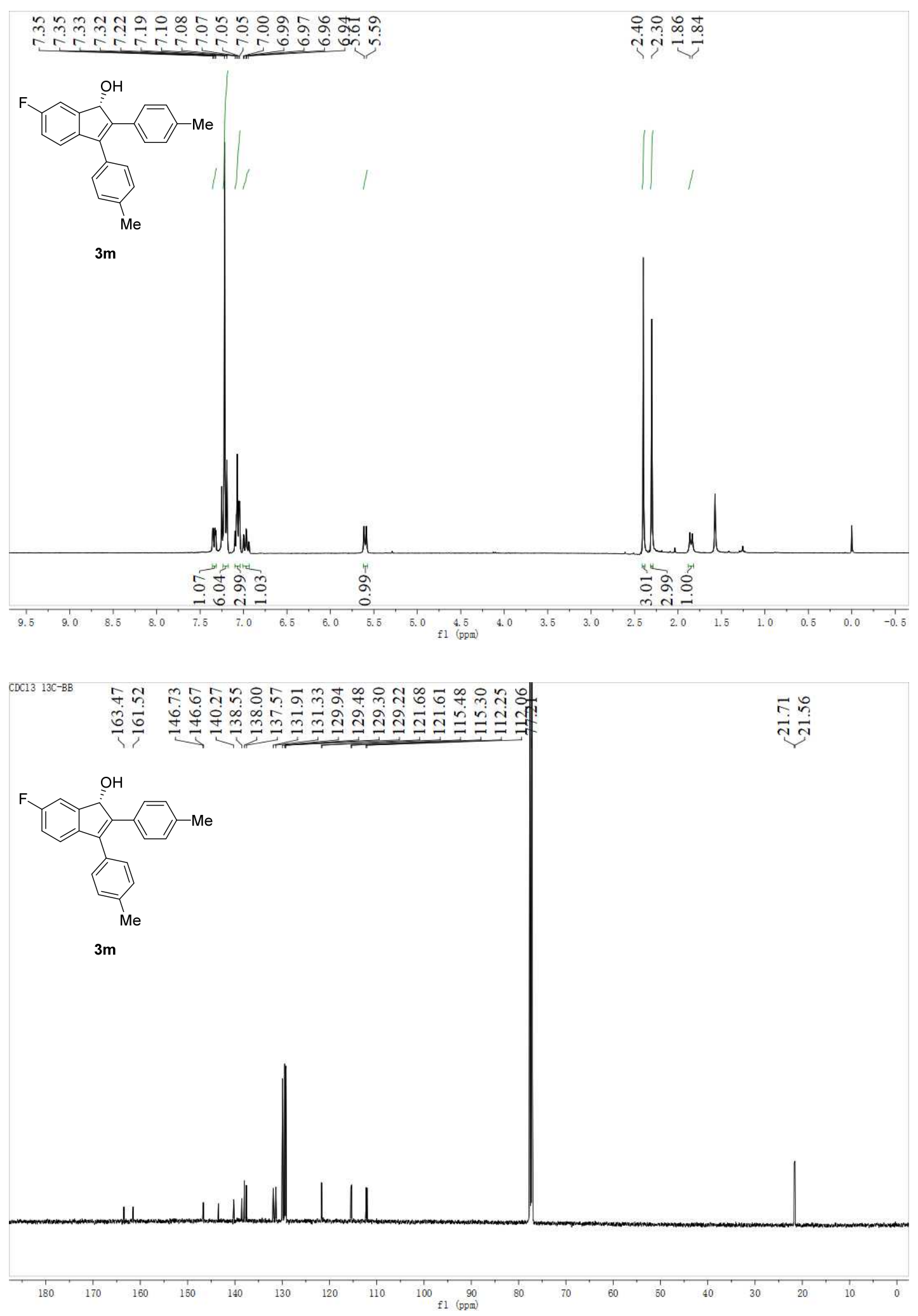

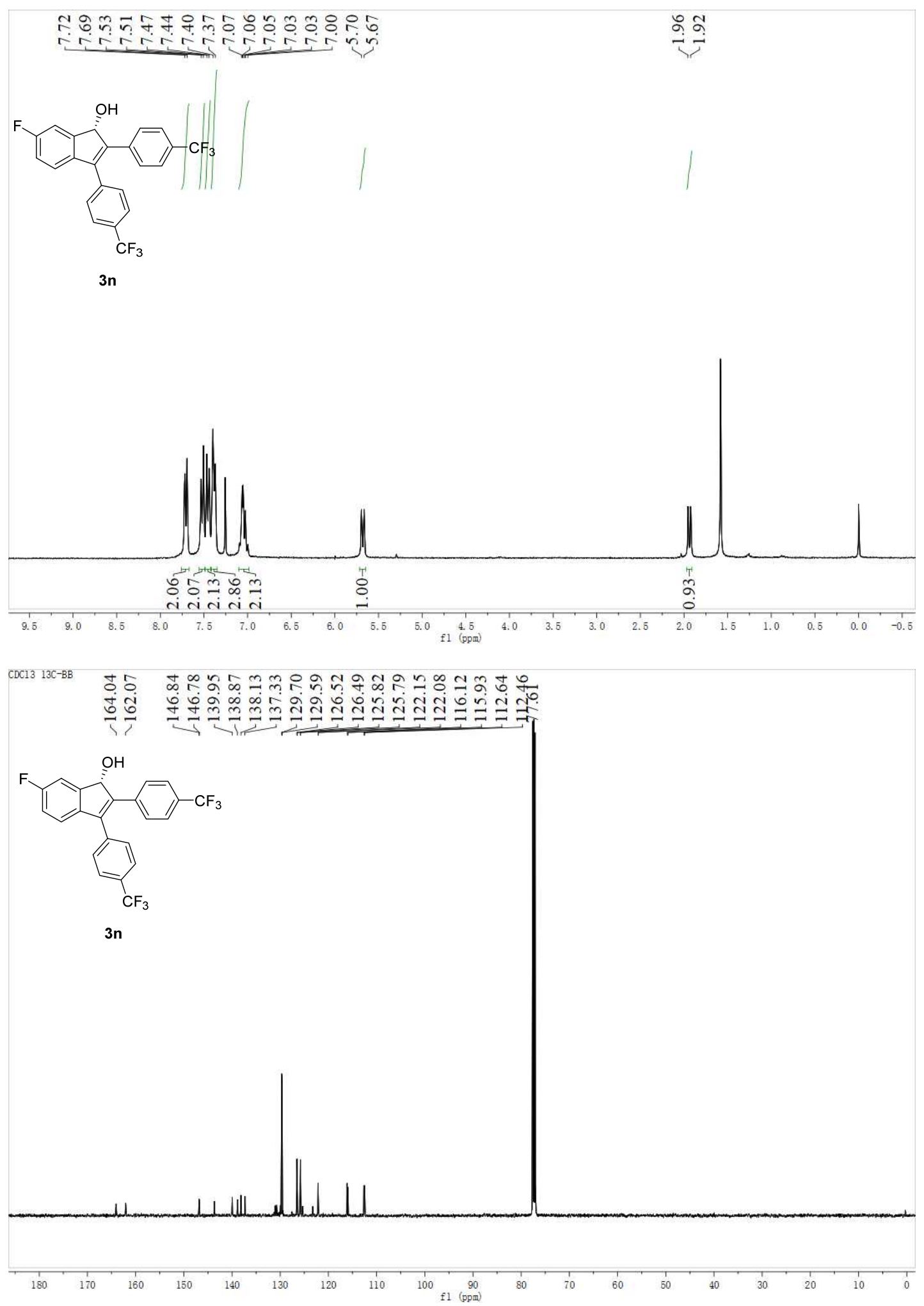

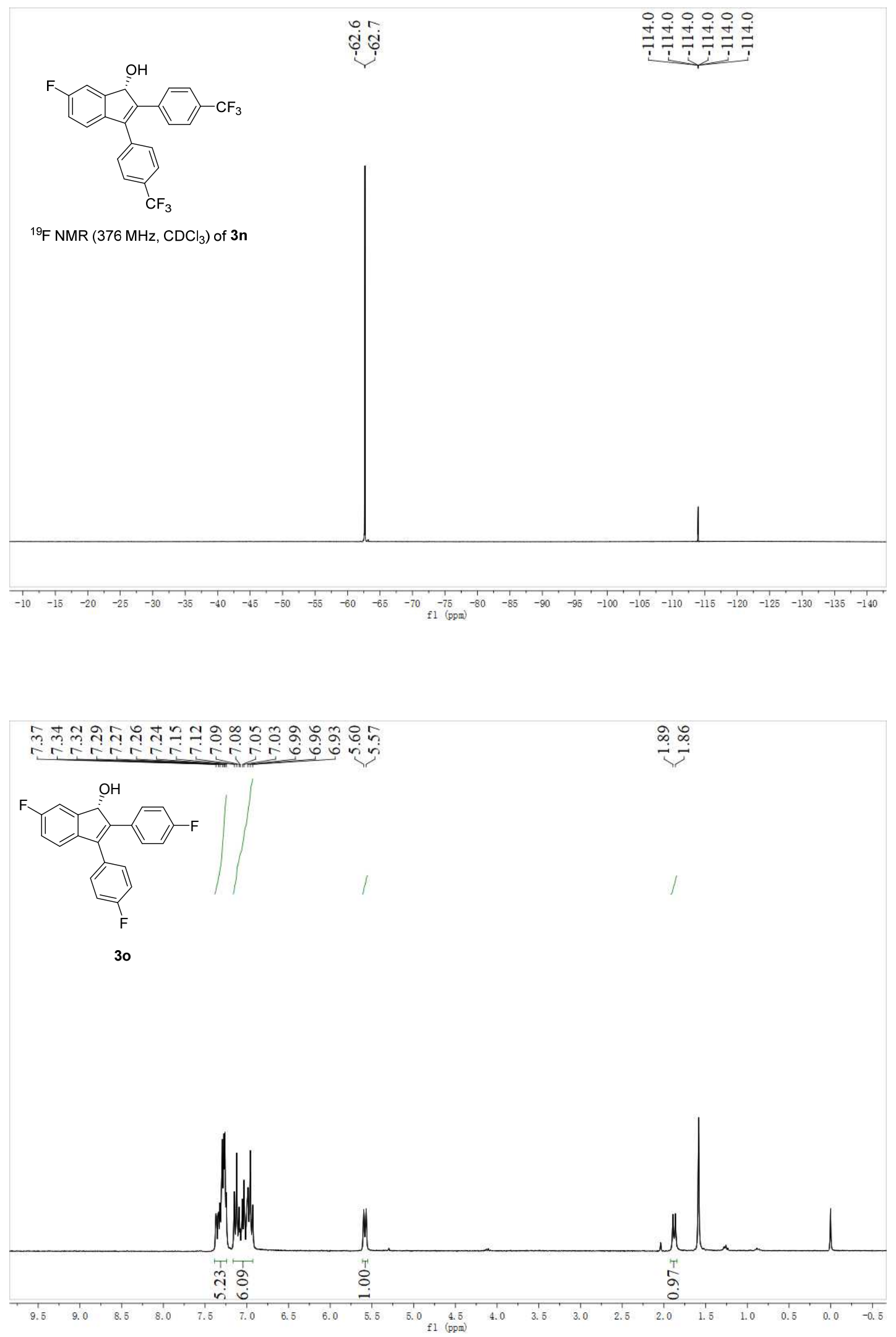

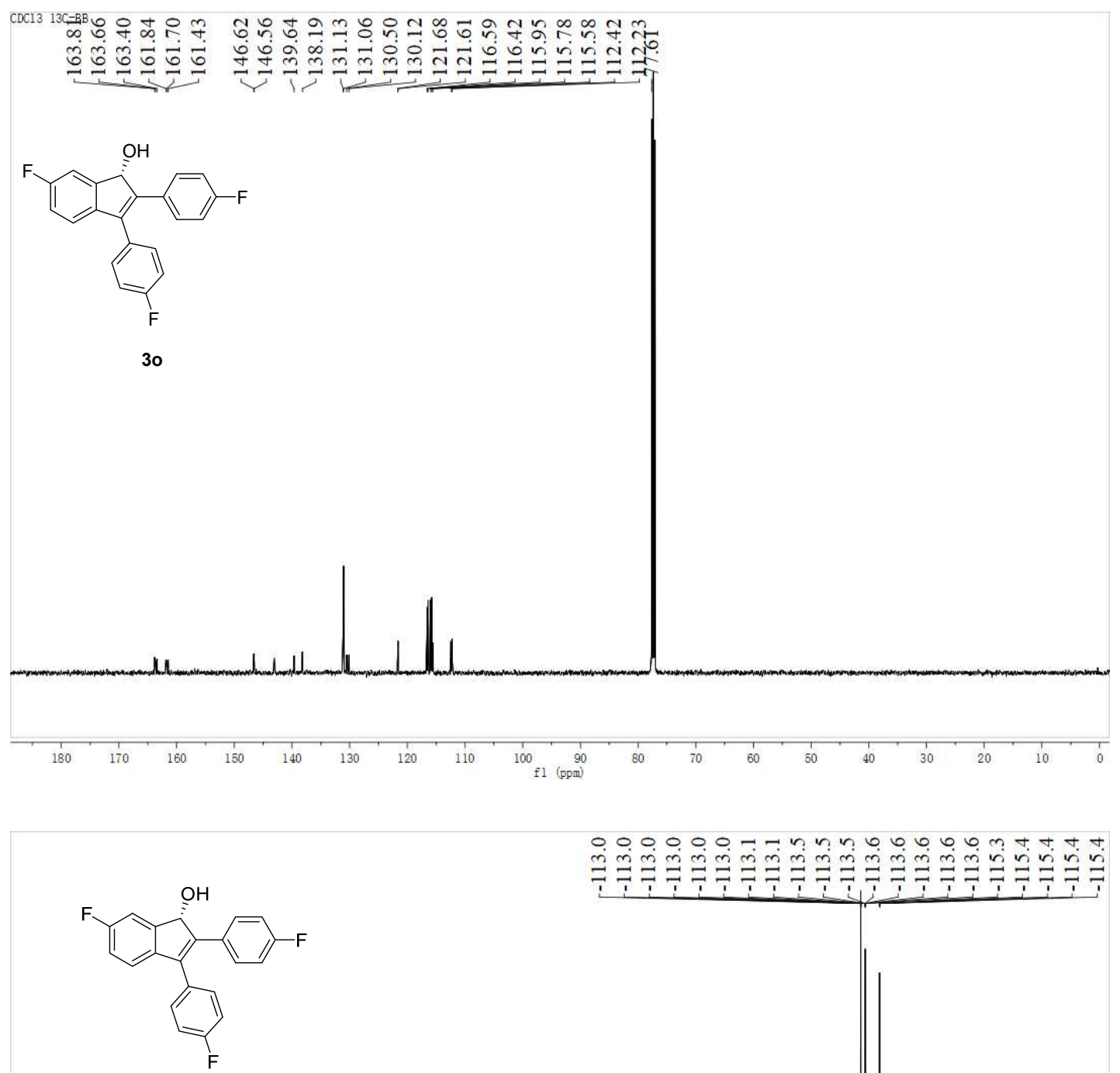

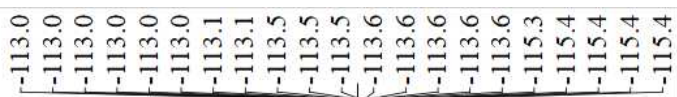

${ }^{19} \mathrm{~F} \mathrm{NMR}\left(376 \mathrm{MHz}, \mathrm{CDCl}_{3}\right)$ of 30

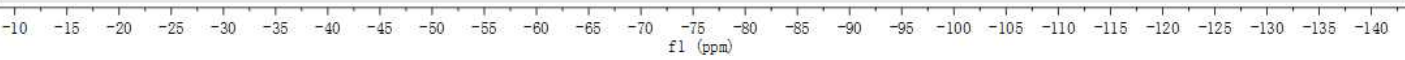



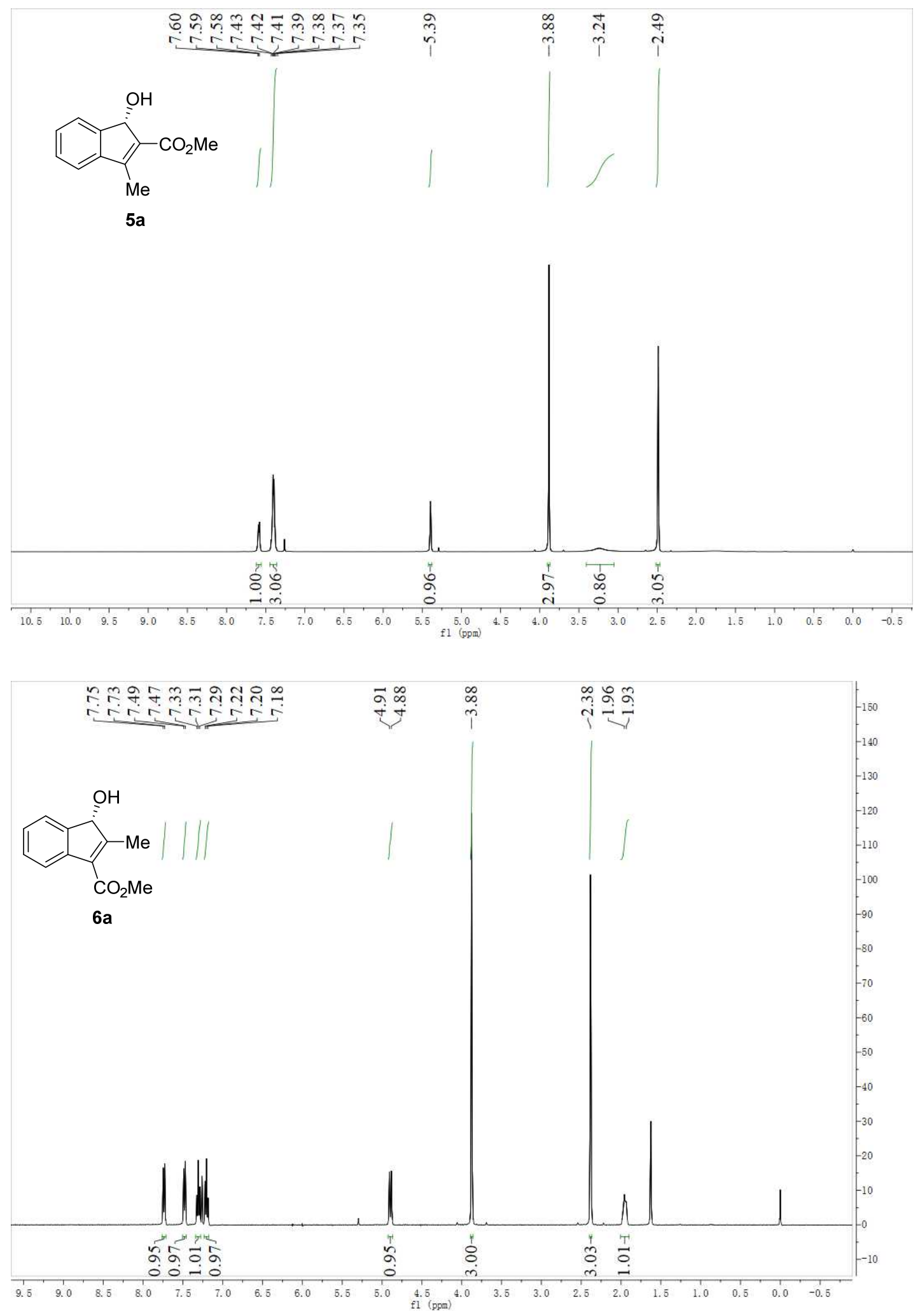

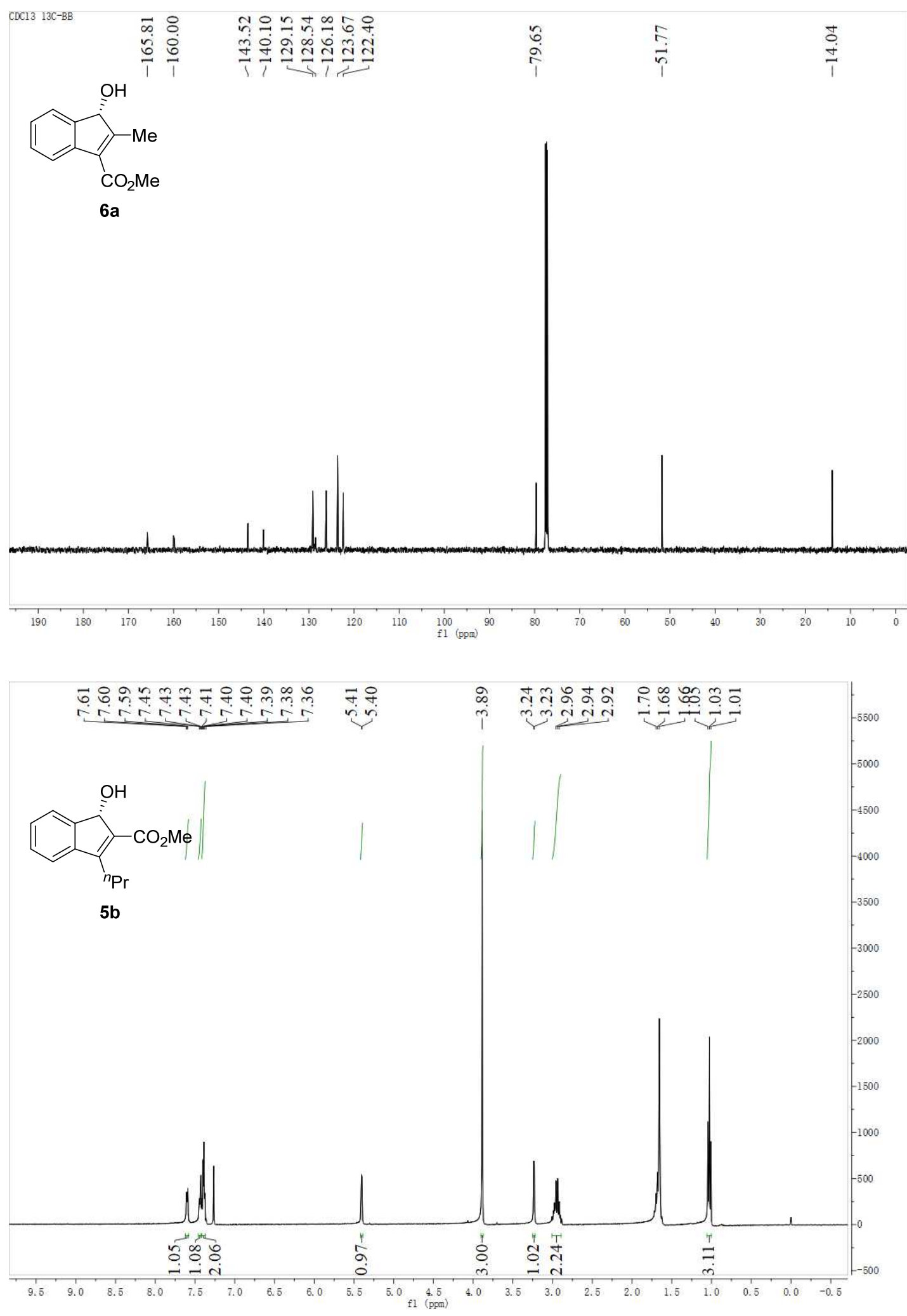

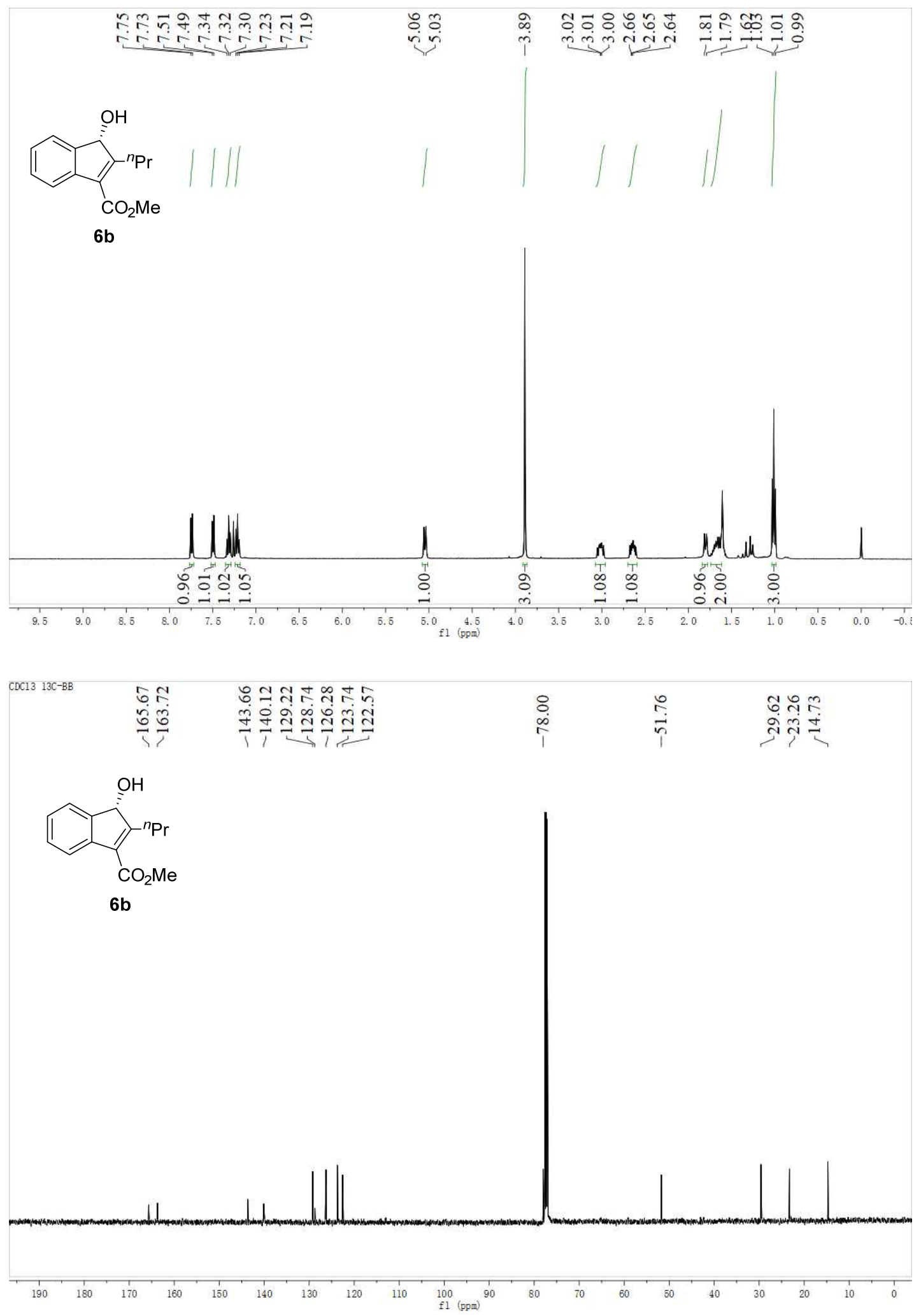

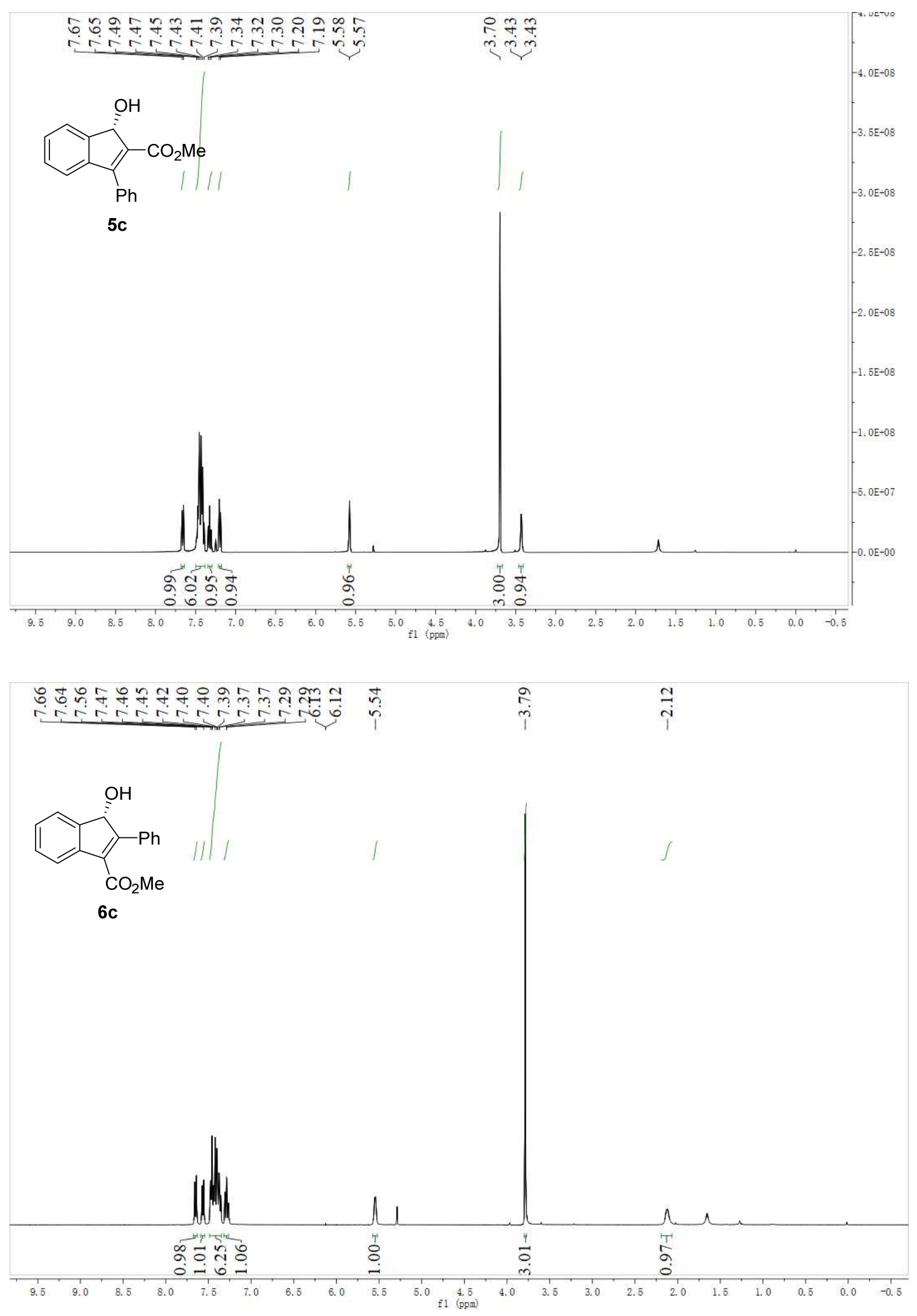

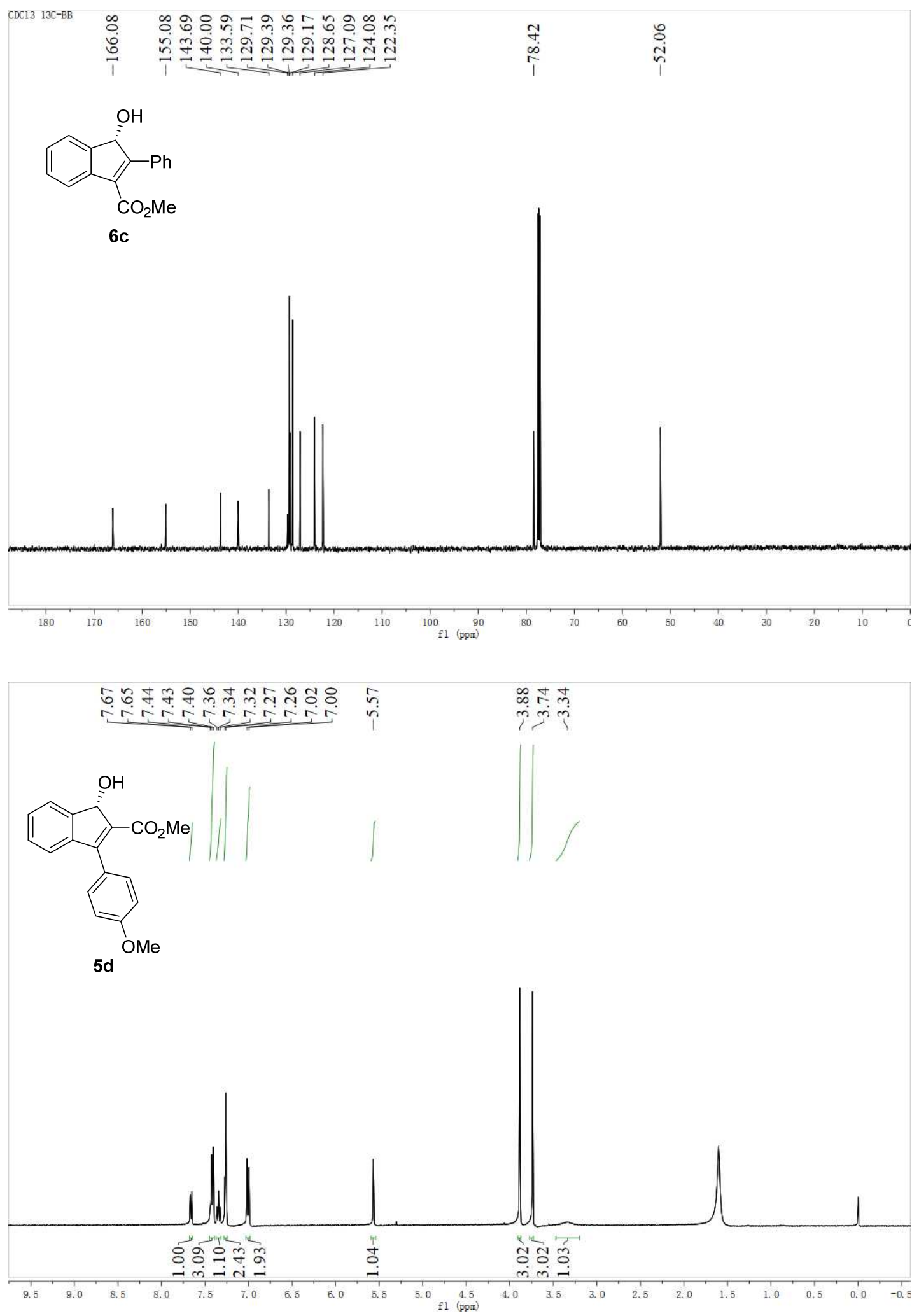

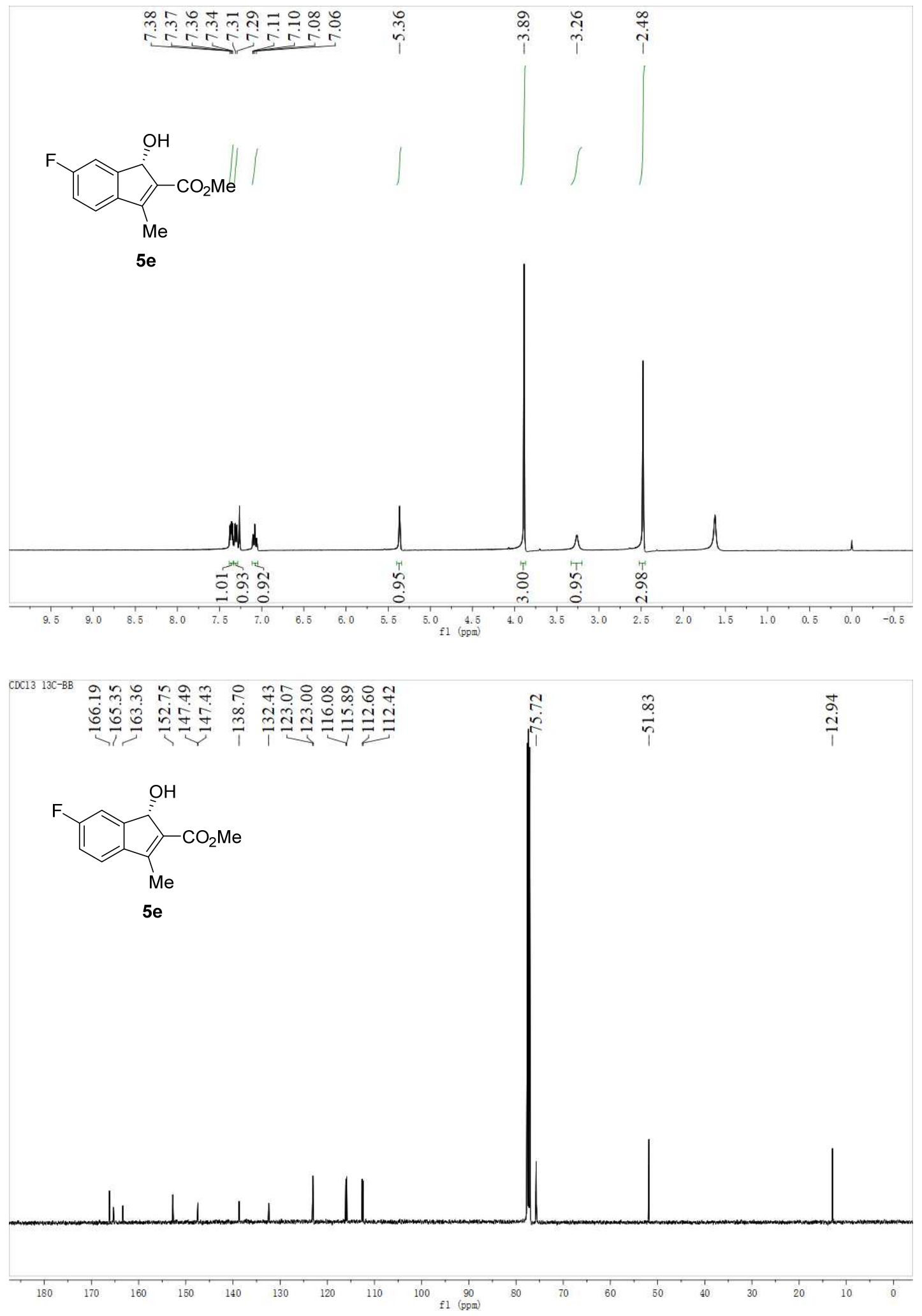


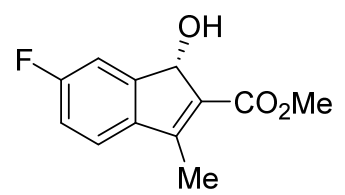

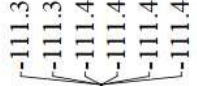

$\left.{ }^{19} \mathrm{~F} \mathrm{NMR} \mathrm{(376} \mathrm{MHz,} \mathrm{CDCl}_{3}\right)$ of $\mathbf{5 e}$
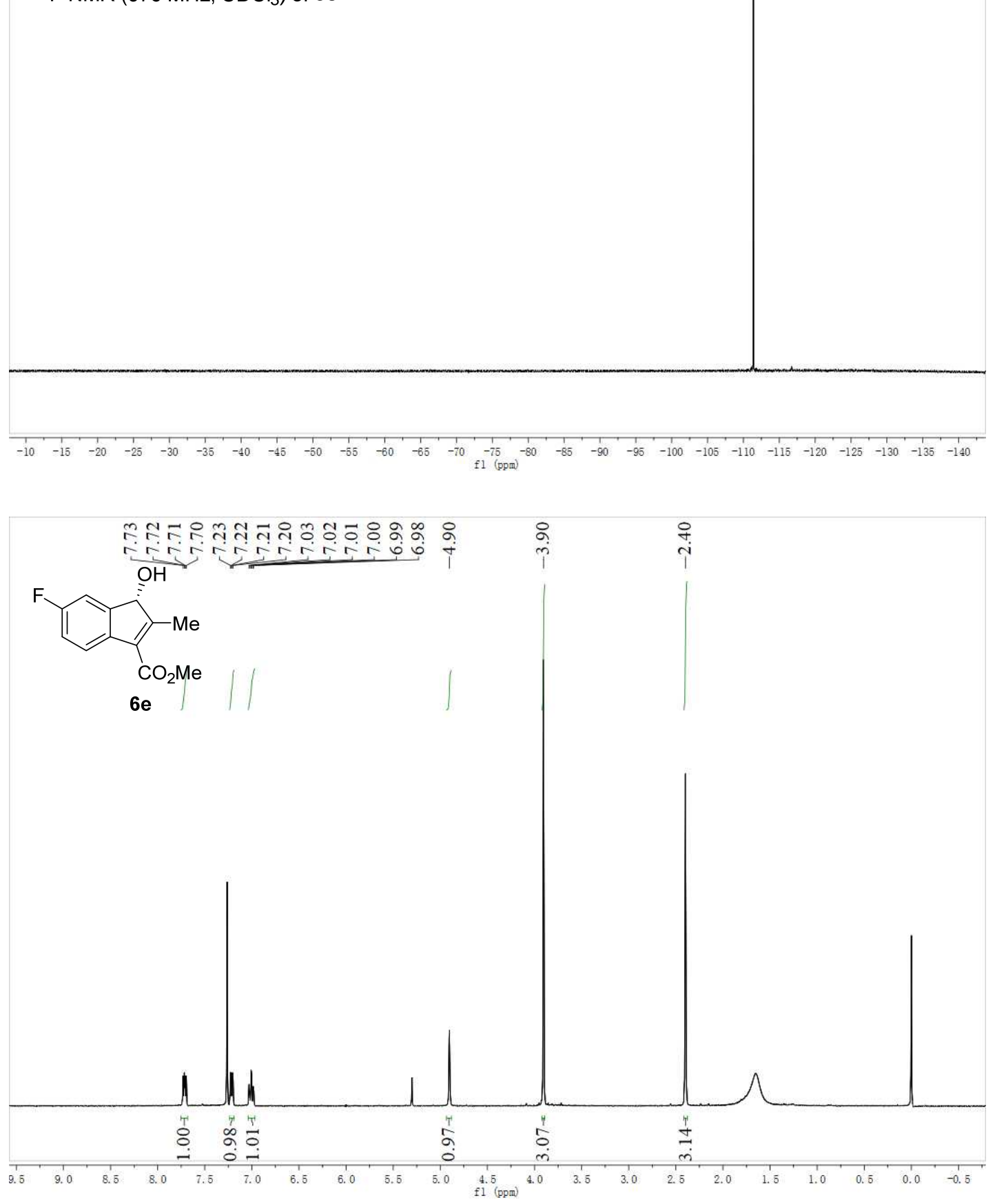

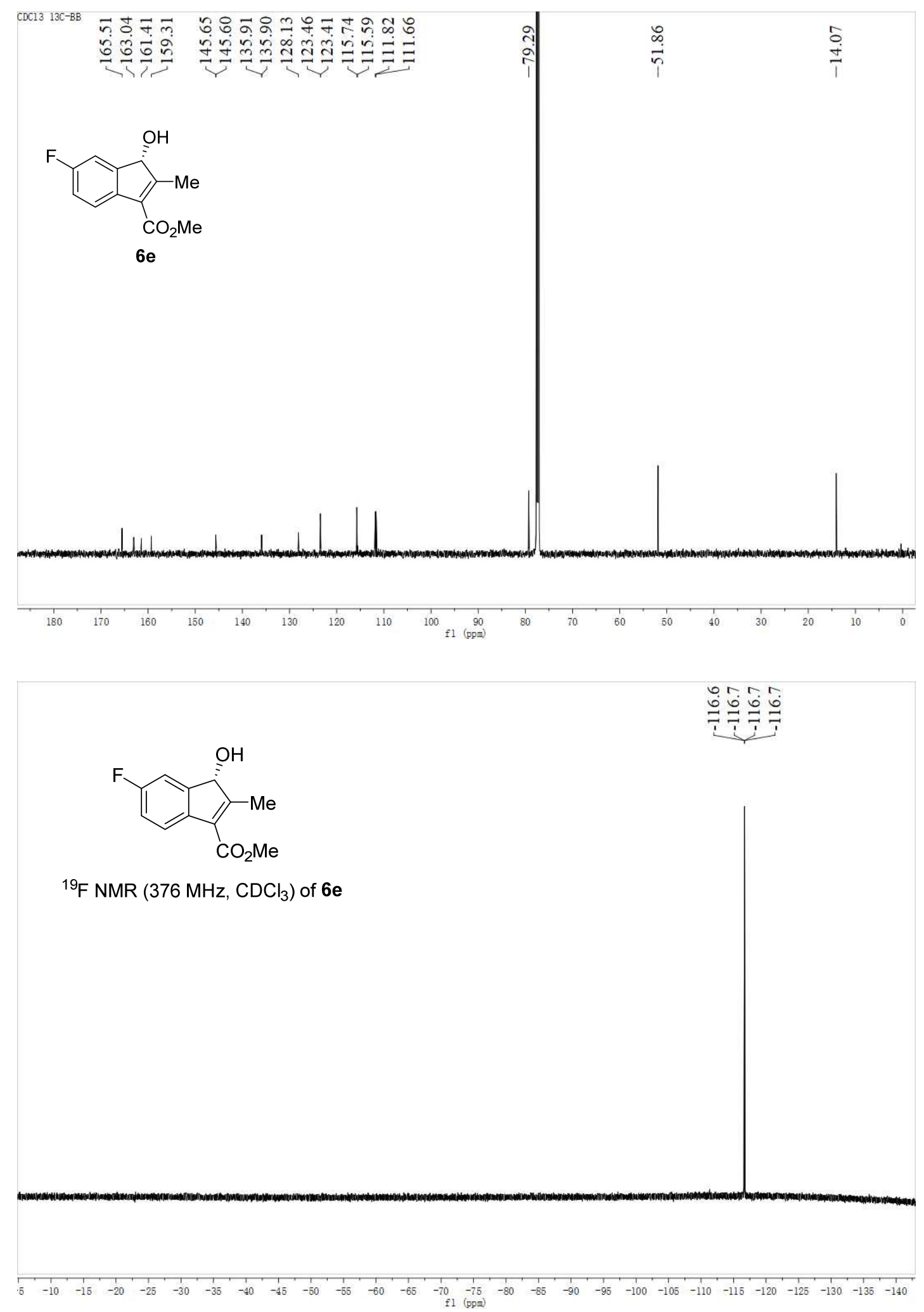

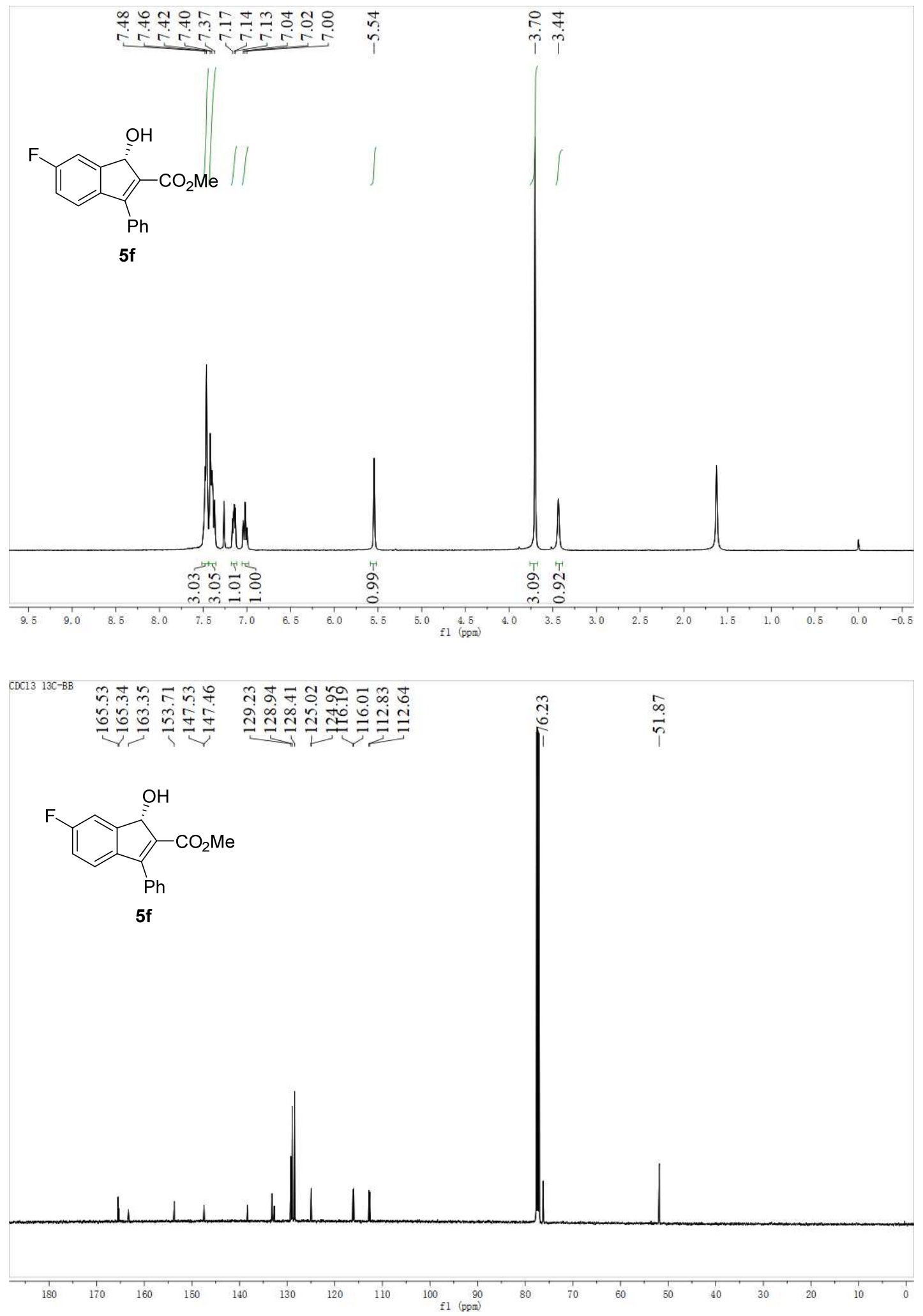

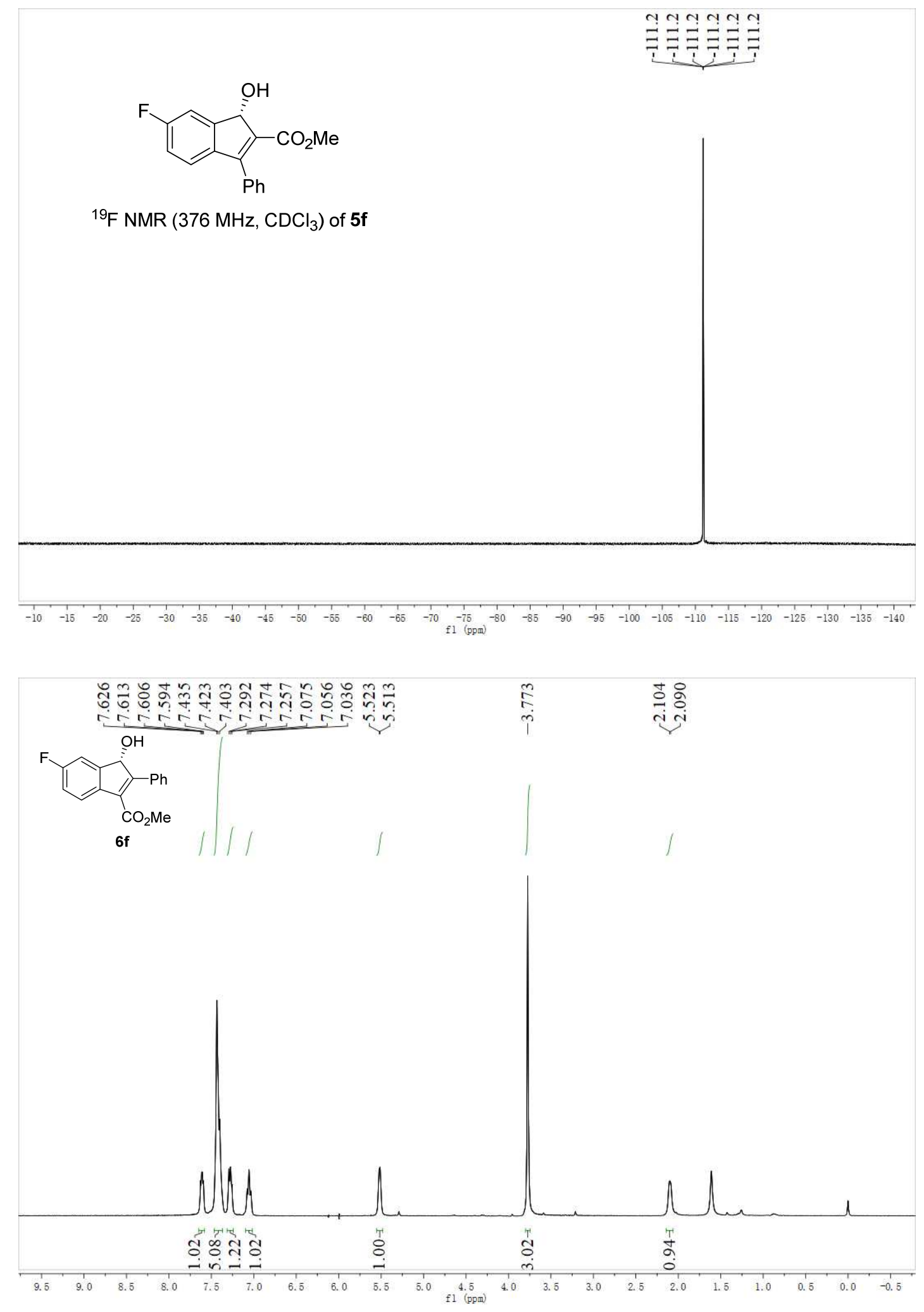


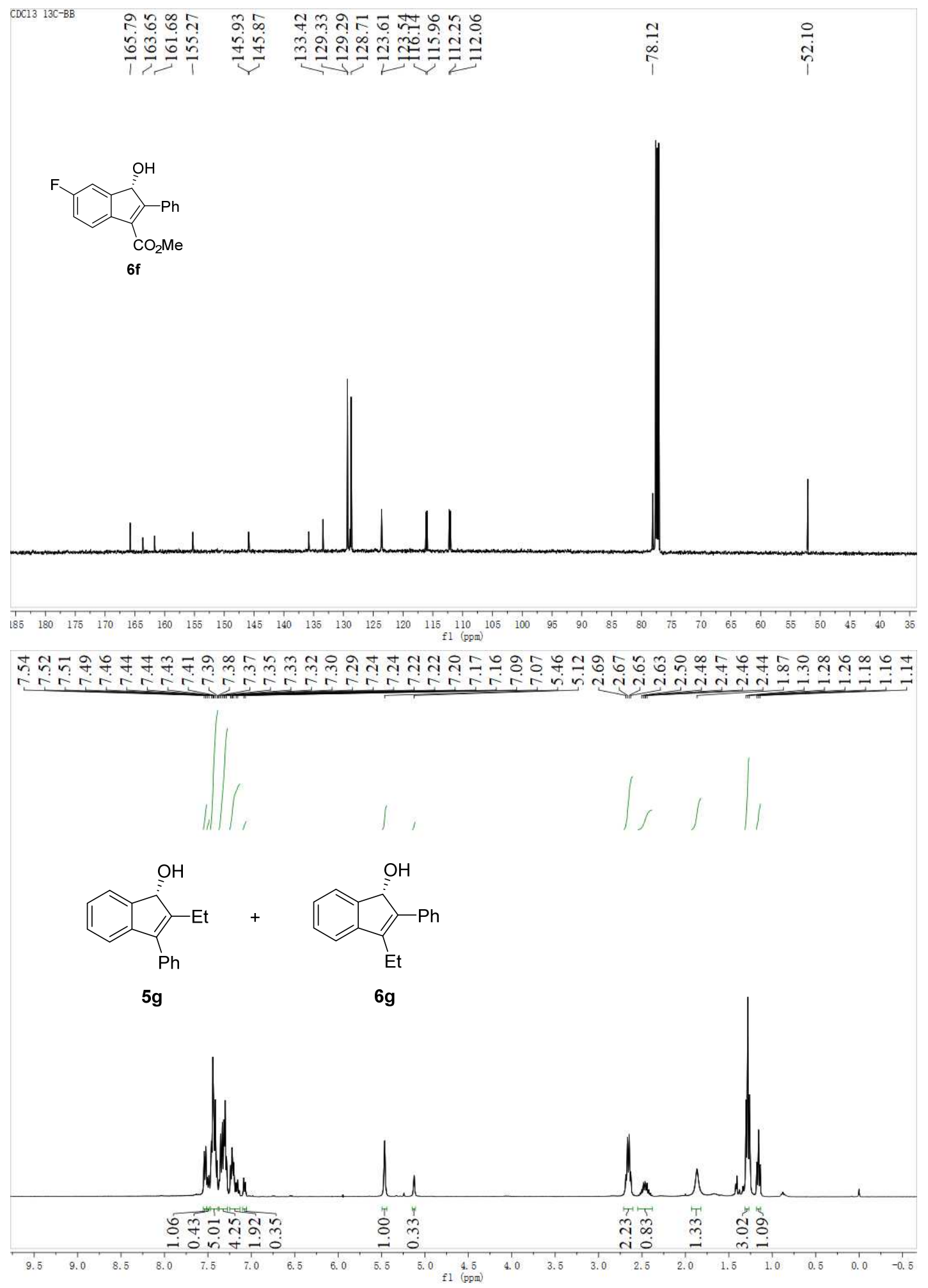



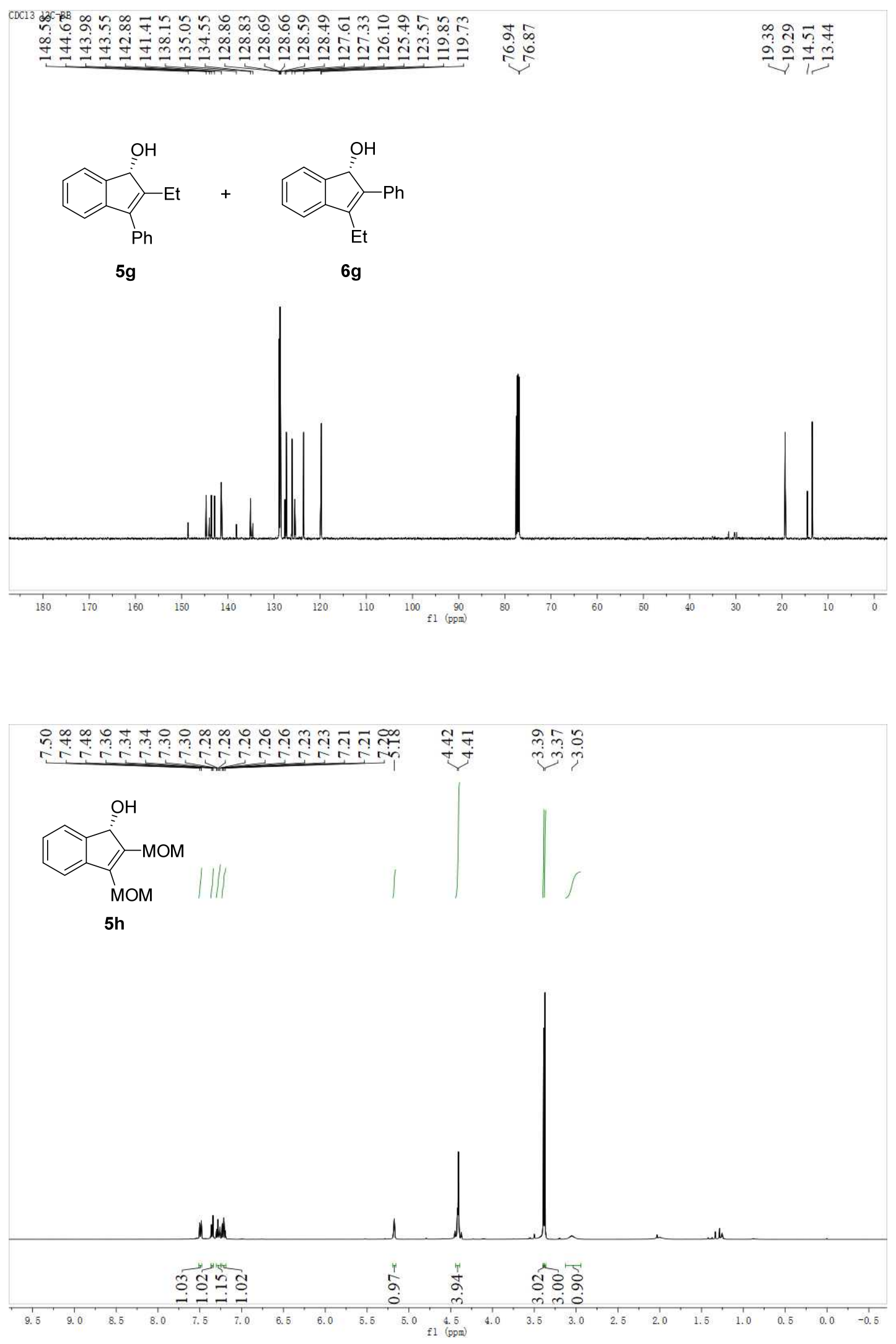


\section{X-Ray structure of compound 3k}<smiles>O[C@H]1C(c2ccccc2)=C(c2ccccc2)c2ccc(F)cc21</smiles>

3k

(CCDC No. - 1962525)

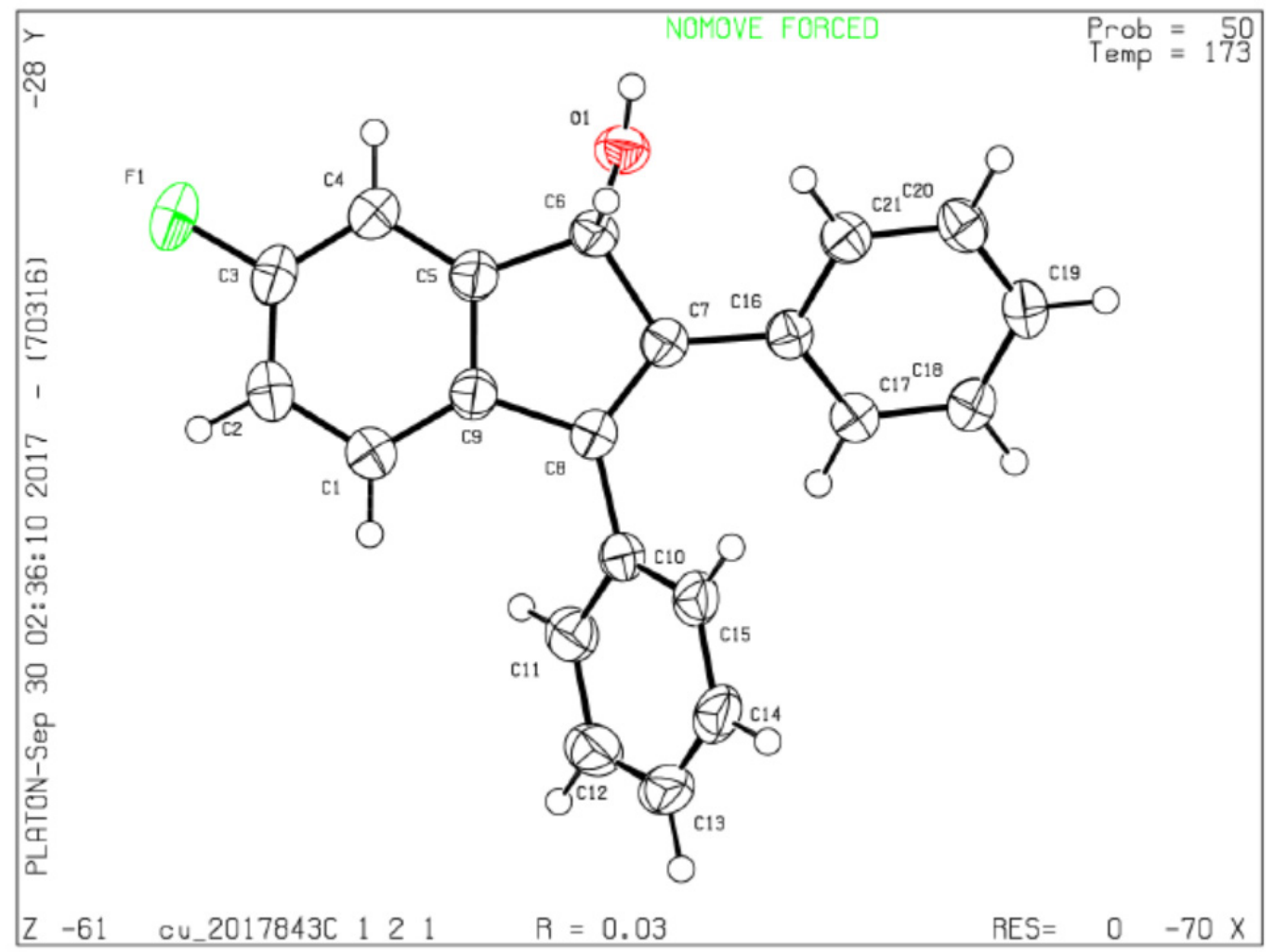

Single crystals suitable for X-ray diffraction analysis were obtained by vapor diffusion experiment: compound 3k $(10 \mathrm{mg})$ was dissolved in $2.0 \mathrm{~mL}$ of DCM in a $10 \mathrm{~mL}$ glass vial. Then the vial was sealed, standing quietly. Crystals were obtained in about 1-2 days. The absolute structure of the compound $\mathbf{3 k}$ was established to be $S$ by X-ray diffraction studies. The compound crystallizes in the space group C121 with unit cell parameters: $a=27.769(2), b=5.4256(4), c=11.0130(8) \AA$, and $Z=4$. The crystal structure was solved by direct methods using single-crystal X-ray diffraction data and refined to $\mathrm{R}=$ 0.0326 for 2316 observed reflections.

CCDC-1962525 contains the supplementary crystallographic data for this paper. These data can be obtained free of charge from The Cambridge Crystallographic Data Centre via www.ccdc.cam.ac.uk/data_request/cif. 


\section{Datablock: cu_2017843_0m}

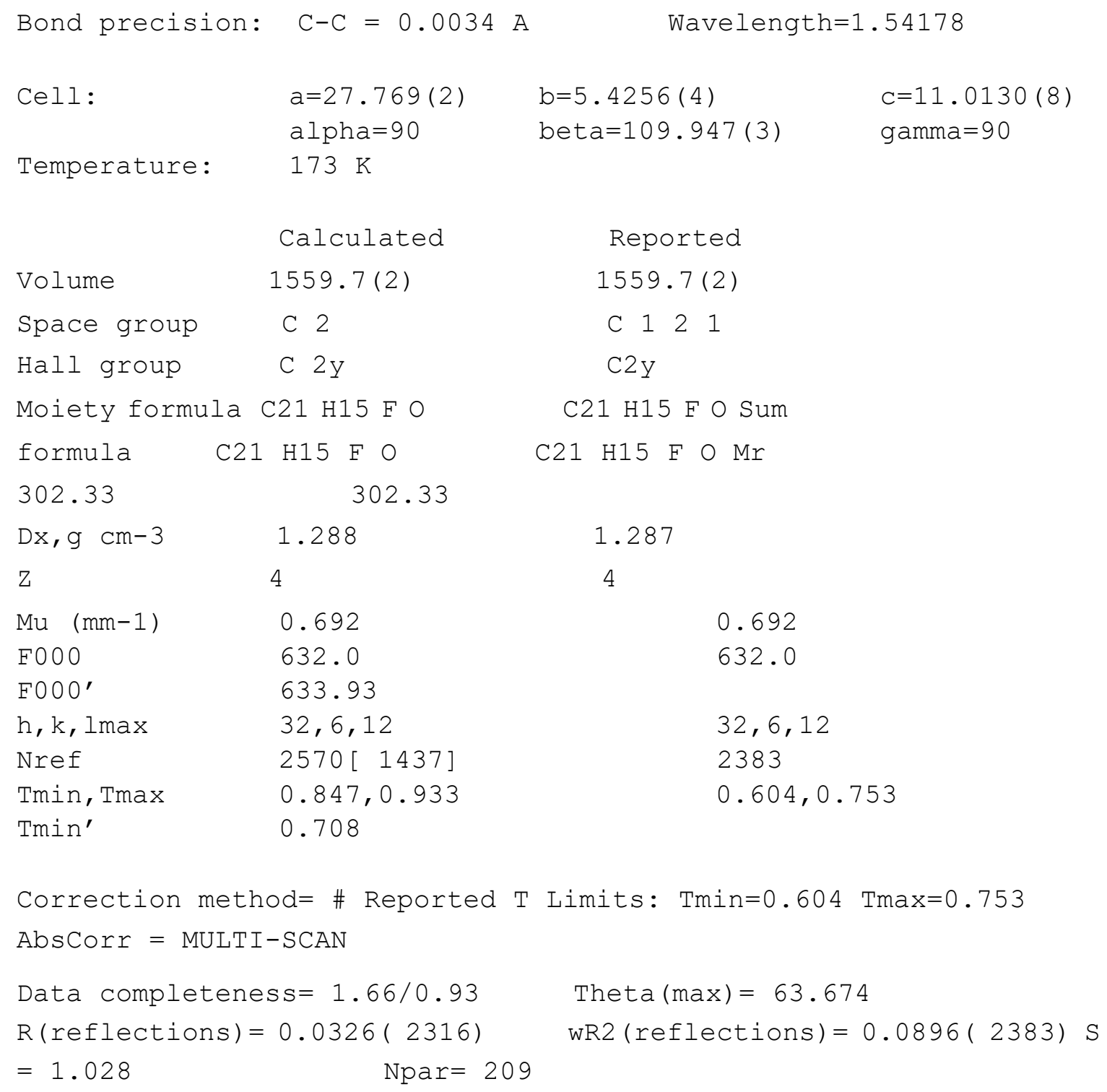

